\title{
An annotated checklist of vascular plants of Cherangani hills, Western Kenya
}

\author{
Yuvenalis Morara Mbuni1 ${ }^{1,2,4, *}$, Yadong Zhou ${ }^{1,3, *}$, Shengwei Wang ${ }^{1,2}$, \\ Veronicah Mutele Ngumbau ${ }^{1,2,4}$, Paul Mutuku Musili ${ }^{4}$, Fredrick Munyao Mutie 1,2,4, \\ Brian Njoroge ${ }^{1,2,4}$, Paul Muigai Kirika ${ }^{4}$, Geoffrey Mwachala ${ }^{4}$, Kathambi Vivian ${ }^{1,2,4}$, \\ Peninah Cheptoo Rono ${ }^{1,2,4}$, Guangwan $\mathrm{Hu}^{1,3}$, Qingfeng Wang ${ }^{1,3}$
}

I Wuhan Botanical Garden, Chinese Academy of Sciences, Wuhan 430074, Hubei, PR China 2 University of Chinese Academy of Sciences, Beijing 100049, PR China 3 Sino-Africa Joint Research Center (SAJOREC), Chinese Academy of Sciences, Wuhan 430074, Hubei, PR China 4 East African Herbarium, National Museums of Kenya, P. O. Box 4516600100 Nairobi, Kenya

Corresponding authors: Guangwan Hu (guangwanhu@wbgcas.cn); QingfengWang (qfwang@wbgcas.cn)

Academic editor: M. Muasya | Received 3 October 2018 | Accepted 22 January 2019 | Published 18 April 2019

Citation: Mbuni YM, Zhou Y, Wang S, Ngumbau VM, Musili PM, Mutie FM, Njoroge B, Kirika PM, Mwachala G, Vivian K, Rono PC, Hu G, Wang Q (2019) An annotated checklist of vascular plants of Cherangani hills, Western Kenya. PhytoKeys 120: 1-90. https://doi.org/10.3897/phytokeys.120.30274

\begin{abstract}
Cherangani hills, located in Western Kenya, comprises of 12 forest blocks, maintaining great plant diversity. However, little attention to plant diversity studies has been paid to it in the past years. Here, we present a checklist of the vascular plants of this region obtained through intensive field investigations and matching of herbarium specimens. In total, 1296 species, including 17 endemic species are documented, belonging to 130 families and 608 genera. This flora represents $18.50 \%, 43.83 \%$ and $54.17 \%$ of the Kenyan species, genera and families, respectively. The habit, habitat and voucher specimens, as well as brief notes on the distribution of each taxon recorded are presented in this checklist. It is the first exhaustive inventory of the terrestrial vascular plants in Cherangani hills which is a significant regional centre for plant diversity.
\end{abstract}

\section{Keywords}

Checklist, Cherangani hills, vascular plants, diversity, endemism

\footnotetext{
${ }^{*}$ Contributed equally as the first authors.
} 


\section{Table of contents}

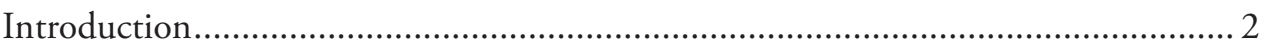

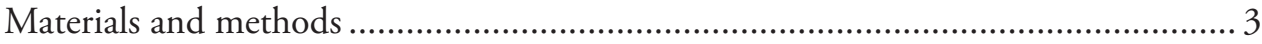

Study site and current vegetation status............................................................. 3

Specimen collection and identification ........................................................ 5

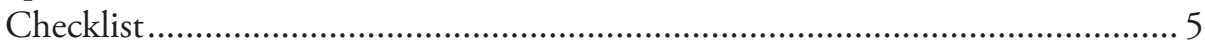

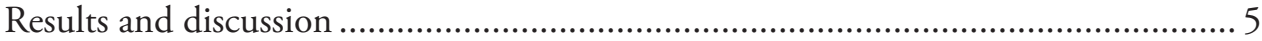

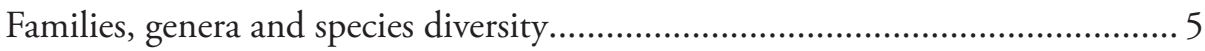

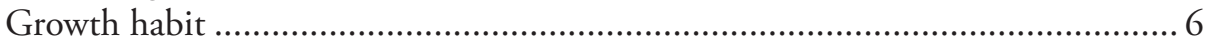

Endemic and threatened species ................................................................... 7

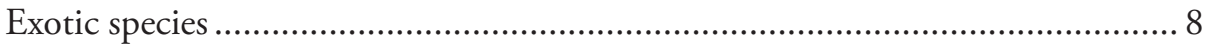

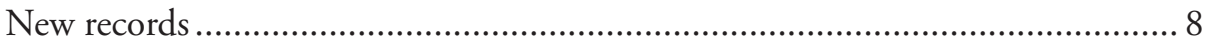

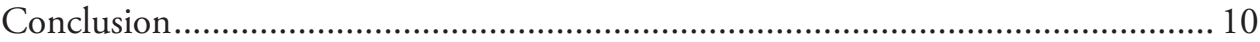

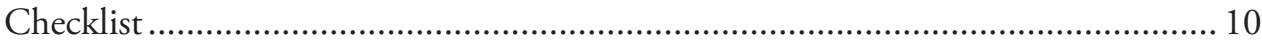

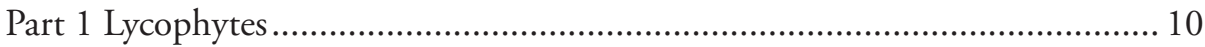

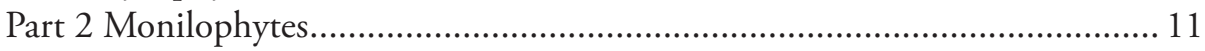

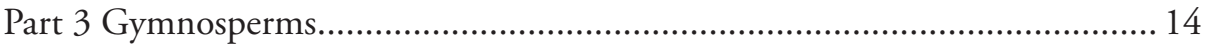

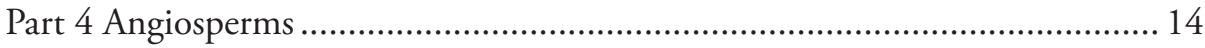

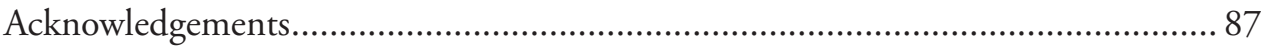

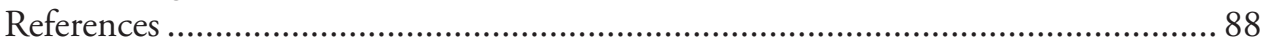

\section{Introduction}

One of the most critical issues on the global, regional and national agenda is the need to preserve biodiversity for future generations and concurrently strive to understand the indigenous knowledge of resource management practices (Nehal et. al. 2004). The concern for biodiversity preservation by government has highlighted the importance derived from broad and small scale vegetation (Whittaker et al. 1979; Frood and Calder 1987; Lunt 1990). Well managed forests are very useful in biodiversity conservation (Sayer et al. 1995). Conserving plant species of special interest in the tropical forest ecosystems ensures that the vital socio-economic and environmental services (e.g. soil and water conservation and wood and non-wood products) are in constant supply to the local communities living near these forests.

Much of the world's terrestrial plant diversity occurs in the tropical forests, where they are facing rapid deforestation (FAO 1997). Agricultural farming increased by $200 \%$ at the expense of the tropical forests between 1900 and 1990 (UNEP 2009a). Currently, many forests in the tropics are facing a myriad of threats including: unsustainable harvesting practices, illegal encroachment and degradation by the dependent communities (UNDP 2006). Over the last 20 years, the local inhabitants have encroached upon the forest land, converting it into settlement and farmlands (UNEP 2009a). Other factors contributing to the forest loss include change of ecological processes due to climate 
change, resulting in alteration of biogeochemical cycles and potential species loss (Wang et al. 2001). Moreover, the rapidly increasing human population is the main reason for the large forest loss in East Africa (Lung and Schaab 2009). In addition, ineffective forest policy implementation has also led to the biodiversity loss in Kenya.

The main goal of a checklist is the provision of high-quality baseline data of a given area or forest on all the accepted taxa. The collection of species resource information, namely species inventory, is the primary component of the biodiversity catalogue and the easiest to understand and operate (Heywood et al. 1997), with the nomenclature being the chief focus, as it is fundamental in the communication about plants and the key to biodiversity status. The process involves investigation, classification, ordering, quantification, mapping, analysis and synthesis of species information. Around the world, research on check listing of plants has been done or is on-going in various places (Chong et al. 2009). Although numerous botanists have collected plant specimens from Cherangani hills forest, no comprehensive species studies have been undertaken in all forest blocks (Synott 1979). In Kenya, numerous studies on plant checklists covering different regions have been undertaken on various ecological regions. Some of the major plant checklists include, Bura Tana River (Gachathi et al. 1994), Ol Ari Nyiro Range (Muasya et al. 1994), Mount Elgon (Tweedie 1976), Taita hills (Beentje 1988); (Thijs et al. 2013), Mount Nyiru (Bytebier and Bussmann 2000), Shimba hills (Luke 2005), Kakamega forest (Fischer et al. 2010), Nandi Forests (Girma et al. 2015) and Mount Kenya (Zhou 2017). For Cherangani hills forest, few ad hoc expeditions resulted in several herbarium collections but failed to give the actual floristic listing, composition and distribution of the species (Blackett 1994).

The aim of this paper is to provide the first comprehensive checklist of the vascular species of Cherangani hills. The checklist will provide the basis for further studies of the indigenous species. It will also assist in implementing policies and strategies for the ecological research and conservation of this unique Highland forest ecosystem in Western Kenya.

\section{Materials and methods}

\section{Study site and current vegetation status}

Cherangani hills are located in the western side of Kenya within an area defined by $1^{\circ} 16^{\prime} \mathrm{N}, 35^{\circ} 26^{\prime} \mathrm{E}$ and comprises 12 forest blocks, including Kapolet, Cheboyit, Chemurkoi, Embobut, Kaisungor, Kererr, Kipkunurr, Kiptaberr, Sogotio, Toropket, Kapkanyar and Lelan (UNEP 2009a) (Fig. 1). Within Cherangani hills, relatively undisturbed (nearly primary) forest, as well as disturbed, secondary forest, swamps and riverine forest and natural glades are present. Kapkanyar, Kapolet and Kiptaberr blocks compose the western block Forest Reserves and are comprised of bushland (Pratt et al. 1966), which have undisturbed forest ecology and together total approximately 20,000 ha. They are closed forests as there are no indications of human 


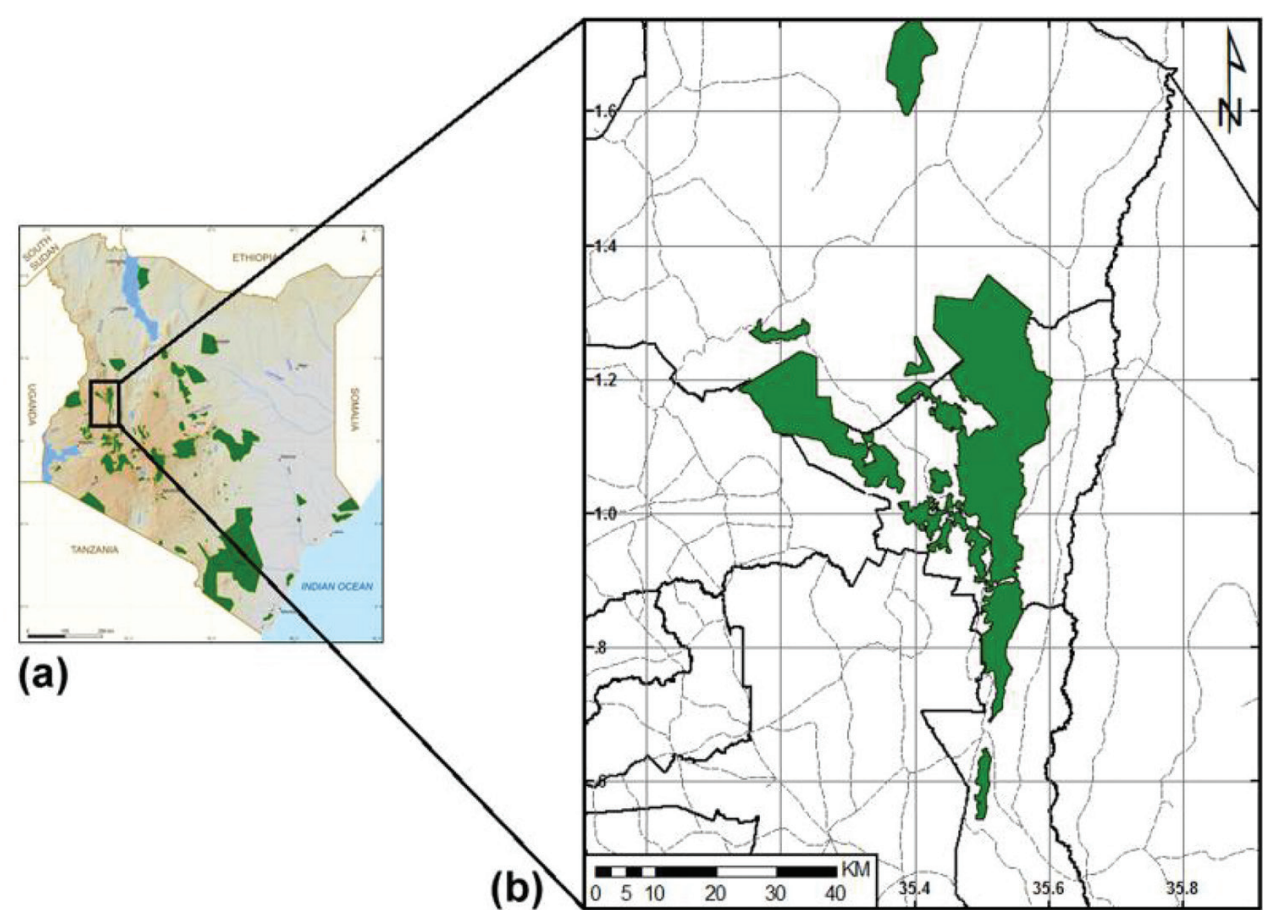

Figure I. The locality of Cherangani hills. a Map of Kenya $\mathbf{b}$ the forest blocks of Cherangani hills.

activity, hence there is a higher plant diversity, which is dominated by AningeriaStrombosia-Drypetes forest, with a large area of mixed Podocarpus latifolius forest on the higher slopes. According to FAO (2010), all terrestrial ecosystems classified as primary forests are characterised as the most species-rich and diverse. This area is therefore classified as closed forest since it has a tree cover of $40 \%$ or more (UNEP et al. 2009b). To the east, forest reserves' blocks of Lelan, Embobut, Kererr, Kaisungor, Toropket, Chemurkoi, Kipkunurr, Cheboyit, Sogotio and Kapchemutwa are also comprised of woodland and grassland which are separate and have been interfered with by humans and domestic animals. Areas with vegetation cover other than forests, but are not under intensive land use, are defined as woodlands and are mostly used for cattle grazing (Holmgren 2006).

The forests on the south-eastern block along the escarpment crest are fragmented and separated by extensive natural grasslands, scrub and farmland especially in the central part. This area holds Juniperus-Nuxia-Podocarpus forest, with heavily disturbed Podocarpus falcatus forest on the eastern slopes. Generally, most of this area consists of middle-aged secondary forest, but much of it is characterised by very young secondary forest. This area is generally highly degraded and fragmented and the composition of plant communities has been greatly influenced by past commercial logging activities and other anthropogenic disturbances (Mitchell and Schaab 2008). Within the valleys in the upper peak areas, remnants of Juniperus-Maytenus undata-Rapanea-Hagenia forest could be found. Cyathea manniana occurs in the stream valleys and there are 
patches of Bamboo (Arundinaria alpina), even though there is no bamboo zone along the Cherangani hills. At higher altitudes, the forest is interspersed with a mixture of heath vegetation and swamps and the latter with Lobelia aberdarica, Senecio johnstonii and Dendrosenecio cheranganiensis. There are different types of vegetation especially in the east, with little obvious altitudinal zonation and an establishment of plantations of Cupressus lusitanica, Pinus patula and a few Eucalyptus species.

\section{Specimen collection and identification}

Between July 2015 and May 2018, the botanical team consisting of botanists from the National Museums of Kenya and Sino-Africa Joint Research Center held several explorations in the Cherangani hills. A combination of general walk-over survey method (Filgueiras et al. 1994) and a plotless landscape defined sampling methods for plant specimen collection and sight observation was used to aid the characterisation of the vegetation (Hall and Swaine 1981). Plant specimens bearing flower or fruit were collected and identified. Details of identified specimens were recorded. Specimen details included habit, habitat, distribution and collector details. The specimens were then preserved by pressing.

Voucher specimens were deposited at the East African Herbarium, Nairobi, Kenya (EA) and Herbarium of Wuhan Botanical Gardens, Wuhan, China), labelled with the plant's scientific name and common names, collection date, GPS coordinates, habitat, collector, identifier and a herbarium specimen number. Standard references were used for plant identification (FTEA 1952-2012; Blundell 1987; Agnew and Agnew 1994; Beentje 1994; Stewart and Campbell 2006; Agnew 2013).

\section{Checklist}

A comprehensive checklist of the vascular plant taxa of the Cherangani hills is enumerated below. Within this checklist, species are organised alphabetically in each family. The families are divided into four groups, namely lycophytes, monilophytes, gymnosperms and angiosperms. Families of lycophytes and monilophytes are organised based on the PPG I system (PPG I 2016), while those of gymnosperms are organised based on Christenhusz et al. (2011) and families of angiosperms are organised based on the APG IV system (APG IV 2016).

\section{Results and discussion}

\section{Families, genera and species diversity}

The current checklist contains a total of 1296 vascular species belonging to 130 families and 608 genera that are indigenous, naturalised or introduced to Cherangani hills. This represents $18.50 \%$ of the total number of 7004 Kenyan vascular species obtained, 
Table I. The 10 largest families and genera of the vascular plants of Cherangani hills.

\begin{tabular}{lcc|lcc}
\hline \multicolumn{1}{c}{ Family } & Species & Genera & \multicolumn{1}{c}{ Genera } & Family & Species \\
\hline Asteraceae & 165 & 66 & Helichrysum & Asteraceae & 20 \\
Fabaceae & 98 & 39 & Cyperus & Cyperaceae & 19 \\
Lamiaceae & 68 & 32 & Plectranthus & Lamiaceae & 17 \\
Orchidacea & 67 & 23 & Crotalaria & Fabaceae & 14 \\
Rubiaceae & 64 & 25 & Vernonia & Asteraceae & 13 \\
Poaceae & 59 & 40 & Euphorbia & Euphorbiaceae & 13 \\
Cyperaceae & 40 & 09 & Solanum & Solanaceae & 13 \\
Apocynaceae & 36 & 20 & Carex & Cyperaceae & 12 \\
Acanthaceae & 36 & 17 & Justicia & Acanthaceae & 10 \\
Malvaceae & 34 & 10 & & & \\
\hline
\end{tabular}

$43.83 \%$ of the total number of 1387 Kenyan genera and $54.17 \%$ of the total number of 240 Kenyan families (MEWNR and RDA 2015). However, the number of species is still increasing because of the discovery of new records, new naturalised species and the introduction of invasive species.

The top 10 species-rich families are Asteraceae (66 genera/165 species), Fabaceae (39/98), Labiatae (32/68), Orchidaceae (23/65), Rubiaceae (25/65), Poaceae (40/59), Cyperaceae (8/40), Apocynaceae (20/38), Acanthaceae (17/36) and Malvaceae (10/34) (Table 1). Fabaceae, Poaceae and Asteraceae are amongst the three largest families in Cherangani hills and are also the largest families in the world, having 745 genera/ 19560 species, 707 genera/11337 species and 1620 genera/23600 species, respectively (Stevens 2012) and also the largest families in Kenya, having 98 genera/576 species, 137 genera/565 species and 99 genera/ 403 species (Zhou et al. 2017a). The 9 most speciesrich genera are Helichrysum (20, Asteraceae), Cyperus (19, Cyperaceae), Plectranthus (17, Lamiaceae), Crotalaria (14, Fabaceae), Vernonia (13, Asteraceae), Euphorbia (13, Euphorbiaceae), Solanum (13, Solanaceae), Carex (12, Cyperaceae) and Justicia (10, Acanthaceae) (Table 1).

\section{Growth habit}

The growth habit of Cherangani hills signifies that herbaceous species have the highest percentage of $54.39 \%$. Herbs are plants without persistent woody stems. Shrubs are represented with $23.76 \%$ and can be defined as self-supporting woody plants branching at or near the ground or with several stems from the base or a single stem less than $2 \mathrm{~m}$. A climber is also defined as a plant that grows upwards by attaching itself to other structures which it uses as support. A tree is a perennial woody plant with a secondary thickening, with a clear main trunk, is dependent on the single trunk and has a height of more than $3 \mathrm{~m}$ (Beentje 2016). Trees are also represented with $11.57 \%$ of the total species (Table 2). Plant species richness is usually higher in the herbaceous layer than in any other forest stratum. It is often stated that most plant biodiversity in the forest 
ecosystems is found in the herbaceous layer (Roberts 2004; Whigham 2004), which concludes that herbaceous flora in Cherangani hills has invaded most of the areas and hence has the highest percentage contributing to the high plant diversity.

\section{Endemic and threatened species}

There are 17 endemic plant species in Cherangani hills (Source FTEA), belonging to 5 families and 13 genera and accounting for $1.23 \%$ of the total species of which most are from the herb layer (Table 3). The region is not well protected, hence poses great danger to these species, for example, Dendrosenecio cheranganiensis is classified as a vulnerable species by IUCN (2013). In total, there are 34 threatened plant species (Source FTEA), belonging to 17 families and 28 genera representing 2.62\% of the total plants in Cherangani hills (Table 4). Over-exploitation and habitat loss have led to species becoming rare and threatened, for example, Polyscias kikuyuensis is highly valued for its excellent timber, hence highly extracted.

Table 2. Growth habit of the plants of Cherangani Hills.

\begin{tabular}{|c|c|c|}
\hline Habit & No. of species & Percentage \\
\hline Trees & 150 & $11.57 \%$ \\
\hline Shrubs & 308 & $23.76 \%$ \\
\hline Climbers/Lianas & 81 & $06.25 \%$ \\
\hline Herbs & 705 & $54.39 \%$ \\
\hline Epiphytes & 46 & $3.55 \%$ \\
\hline Parasitic herb & 6 & $0.46 \%$ \\
\hline Total & 1296 & $100 \%$ \\
\hline
\end{tabular}

Table 3. The endemic plants of Cherangani hills.

\begin{tabular}{clc}
\hline \multicolumn{1}{c}{ Family } & \multicolumn{1}{c}{ Endemic species } & Growth habit \\
\hline Acanthaceae & Justicia leikpiensis S. Moore & Herb \\
& Megalochlamys kenyensis Vollesen & Herb \\
Asteraceae & Conyza agnewii Mesfin & Herb \\
& Senecio cheranganiensis Cotton \& Blakelock & Herb \\
& Senecio hedbergii C. Jeffrey & Shrub \\
& Senecio plantagineoides C. Jeffrey & Herb \\
& Senecio pseudosubsessilis C. Jeffrey & Herb \\
& Senecio snowdenii Hutch & Shrub \\
& Kleinia scottioides C. Jeffrey & Herb \\
& Bidens elgonensis (Sherff) Agnew & Herb \\
& Carduus schimperi Sch. Bip. & Herb \\
& Dendrosenecio cheranganiensis (Cotton \& Blakelock) E.B. Knox & Shrub \\
& Emilia tricholepis C. Jeffrey & Herb \\
Moraceae & Helichrysum brownei S. Moore & Shrub \\
Boraginaceae & Dorstenia afromontana R.E. Fr. & Herb \\
Orobanchaceae & Bynoglossum cheranganiense Verdc. & Herb \\
\hline
\end{tabular}


Table 4. The threatened plants of Cherangani hills.

\begin{tabular}{|c|c|}
\hline Family & Species \\
\hline Apiaceae & Peucedanum aculeolatum Engl., Pimpinella lindblomii $\mathrm{H}$. Wolff \\
\hline Araliaceae & Polyscias kikuyuensis Summerh. \\
\hline Asteraceae & $\begin{array}{l}\text { Ethulia vernonioides (Schweinf.) M.G. Gilbert, Euryops brownei S. Moore, Gutenbergia } \\
\text { rueppellii Sch. Bip., Senecio pseudosubsessilis C. Jeffrey, S. rhammatophyllus Mattf., Helichrysum } \\
\text { meyeri-johannis Engl., Guizotia jacksonii (S. Moore) J. Baagøe. }\end{array}$ \\
\hline Balsaminaceae & Impatiens hoehnelii T.C.E. Fr., I. meruensis Gilg, I. pseudoviola Gilg, I. tinctoria A. Rich. \\
\hline Boraginaceae & Cynoglossum cheranganiense Verdc. \\
\hline Campanulaceae & $\begin{array}{l}\text { Lobelia aberdarica R.E. Fr. \& T.C.E. Fr., L. cheranganiensis Thulin, L. deckenii (Asch.) } \\
\text { Hemsl., L. duriprati T.C.E. Fr., Wahlenbergia scottii Thulin }\end{array}$ \\
\hline Euphorbiaceae & Euphorbia brevicornu Pax \\
\hline Fabaceae & Trifolium cheranganiense J.B. Gillett \\
\hline Hypericaceae & Hypericum kiboense Oliv. \\
\hline Leguminosae & Galega lindblomii (Harms) J.B. Gillett \\
\hline Malvaceae & Abutilon mauritianum (Jacq.) Medik. \\
\hline Orchidaceae & $\begin{array}{l}\text { Diaphananthe montana (Piers) P.J. Cribb \& J. Stewart, Habenaria altior Rendle, Polystachya } \\
\text { bella Summerh. }\end{array}$ \\
\hline Poaceae & Calamagrostis hedbergii Melderis \\
\hline Ranunculaceae & Delphinium macrocentrum Oliv. \\
\hline Rosaceae & Rubus scheffleri Engl. \\
\hline Rubiaceae & Galium kenyanum Verdc. Spermacoce minutiflora (K. Schum.) Verdc. \\
\hline Vitaceae & Cyphostemma cyphopetalum (Fresen.) Desc. ex Wild \& R.B. Drumm. \\
\hline
\end{tabular}

\section{Exotic species}

A total of 47 species of exotic plants, belonging to 42 genera and 21 families, were realised from Cherangani hills (Table 5). Introduced, cultivated or naturalised species represent $3.62 \%$ of the total species of Cherangani hills. The top 4 exotic plant families include: Asteraceae (12 species), Fabaceae (5), Solanaceae (4) and Lamiaceae (3). Out of the 48 plant species introduced, herbs have a higher percentage 63\% (30), followed by shrubs $24 \%$ (11) and then trees $13 \%(6)$. Most of the species have been introduced from South America (14), North America (11), Mexico (10), Central America (5), South Asia (5) and from other regions below 4.

\section{New records}

In the recent years, investigation researchers from Kenya and China have found many new species and several new records in Kenya, including, Sedum keniense (Crassulaceae) (Zhou et al. 2016a), Zehneria subcoraicea (Cucurbitaceae) (Zhou et al. 2016b), Z. longiflora (Cucurbitaceae) (Wei et al. 2017), Cissampelos keniensis (Menispermaceae) (Zhou et al. 2017b) and Adenia angulosa (Ngumbau et al. 2017). So far, no new species from this region has been found, although, Parastriga alectroides (Scrophulariaceae), is recorded for the first time in Kenya, particularly in Cherangani hills. An updated description, comparisons with closely related species and revised conservation status for this species has been described. 
Table 5. The exotic plants of Cherangani hills.

\begin{tabular}{|c|c|c|c|}
\hline $\begin{array}{c}\text { Species } \\
\end{array}$ & Growth Habit & Family & Regions of origin \\
\hline Acanthospermum glabratum (DC.) Wild & Herb & Asteraceae & South America \\
\hline Ageratina adenophora (Spreng.) R.M. King \& H. Rob. & Herb & Asteraceae & Mexico and South America \\
\hline Brassica rapa $\mathrm{L}$. & Herb & Brassicaceae & North America \\
\hline Bryophyllum proliferum Bowie ex Hook. & Herb & Crassulaceae & Madagascar \\
\hline Caesalpinia decapetala (Roth) Alston & Shrub & Fabaceae & India \\
\hline Cestrum aurantiacum Lindl. & Shrub & Solanaceae & North and South America \\
\hline Conyza boranensis (S. Moore) Cufod. & Herb & Asteraceae & North America and East Asia \\
\hline Cupressus lusitanica Mill. & Tree & Cupressaceae & North America \\
\hline Cuscuta campestris Yunck. & Climber & Convolvulaceae & North America \\
\hline Datura stramonium L. & Shrub & Solanaceae & Mexico \\
\hline Desmodium adscendens (Sw.) DC. & Herb & Fabaceae & South America \\
\hline Desmodium uncinatum (Jacq.) DC. & Shrub & Fabaceae & South Africa \\
\hline Erigeron bonariensis $\mathrm{L}$. & Herb & Asteraceae & South America \\
\hline Eucalyptus saligna $\mathrm{Sm}$. & Tree & Myrtaceae & Australia \\
\hline Euphorbia hirta L. & Herb & Euphorbiaceae & North America \\
\hline Galinsoga parviflora Cav. & Herb & Asteraceae & Central and South America \\
\hline Gomphocarpus physocarpus E. Mey. & Herb & Apocynaceae & Australia \\
\hline Grevillea robusta A.Cunn. ex R. Br. & Tree & Proteaceae & Australia \\
\hline Hyptis pectinata (L.) Poit. & Herb & Lamiaceae & Central America \\
\hline Kalanchoe prolifera (Bowie ex Hook.) & Herb & Crassulaceae & Madagascar \\
\hline Lantana camara $\mathrm{L}$. & Shrub & Verbenaceae & South America \\
\hline Lantana trifolia $\mathrm{L}$. & Shrub & Verbenaceae & North America \\
\hline Oxalis latifolia Kunth & Herb & Oxalidaceae & Mexico \\
\hline Passiflora edulis Sims & Climber & Passifloraceae & North America \\
\hline Passiflora subpeltata Ortega & Climber & Passifloraceae & Mexico and Central America \\
\hline Persea americana Mill. & Tree & Lauraceae & Mexico and South America \\
\hline Physalis peruviana $\mathrm{L}$. & Herb & Solanaceae & South America \\
\hline Phytolacca octandra $\mathrm{L}$. & Shrub & Phytolaccaceae & Mexico and Central America \\
\hline Pinus patula Schiede ex Schltdl. & Tree & Pinaceae & Mexico \\
\hline Plectranthus barbatus Andrews & Shrub & Lamiaceae & South Africa \\
\hline Psidium guajava $\mathrm{L}$. & Tree & Myrtaceae & Central and South America \\
\hline Ricinus communis $\mathrm{L}$. & Herb & Euphorbiaceae & India \\
\hline Rubus niveus Thunb. & Shrub & Rosaceae & South Asia \\
\hline Rubus rosifolius Sm. & Herb & Rosaceae & South Asia \\
\hline Rumex acetosella $\mathrm{L}$. & Herb & Polygonaceae & South Asia \\
\hline Salvia leucantha Cav. & Herb & Lamiaceae & Mexico \\
\hline Schkubria pinnata (Lam.) Kuntze & Herb & Asteraceae & South America \\
\hline Senna septemtrionalis (Viv.) H.S. Irwin \& Barneby & Shrub & Fabaceae & Mexico and Central America \\
\hline Senna spectabilis (DC.) H.S. Irwin \& Barneby & Shrub & Fabaceae & Mexico and Central America \\
\hline Sigesbeckia orientalis $\mathrm{L}$. & Herb & Asteraceae & Australia \\
\hline Solanum mauritianum Scop. & Shrub & Solanaceae & South America \\
\hline Sonchus oleraceus (L.) L. & Herb & Asteraceae & Europe and West Asia \\
\hline Tagetes minuta $\mathrm{L}$. & Herb & Asteraceae & South America \\
\hline Tithonia diversifolia (Hemsl.) A. Gray & Herb & Asteraceae & Mexico \& Central America \\
\hline Tridax procumbens (L.) L. & Herb & Asteraceae & West Africa \\
\hline Xanthium strumarium $\mathrm{L}$. & Herb & Asteraceae & North America \\
\hline
\end{tabular}




\section{Conclusion}

Efforts to ensure the conservation of Cherangani forest vegetation habitat should be prioritised with much emphasis on the endangered, rare or endemic species, according to the checklist result. The adopted approach in these studies may help to focus on certain species and the setting of research and conservation priorities. The high proportion of Cherangani endemic species and the geographic rarity component of the flora associated with that of Mount Kenya and Mount Elgon plants, are aspects that makes these habitats interesting. Cherangani hills have not been inventoried exhaustively, including all of its 12 forest blocks. Our study provides the baseline data for further studies on the invasion process and on the patterns of biodiversity. More study approaches may also contribute to the identification of species which are threatened by anthropogenic impacts and therefore require protection. Community-based forest conservation is also suggested to ensure sustainable utilisation of these forests.

\section{Checklist}

The following lists of species include references to the elevation, and where metres are stated, this means metres above sea level. EA means the East African Herbarium, Nairobi, Kenya; HIB means the Herbarium of Wuhan Botanical Garden, Wuhan, China.

\section{Part I Lycophytes}

\section{F1. Lycopodiaceae}

2 Genera, 4 Species

Huperzia dacrydioides (Baker) Pic. Serm. Habit: Epiphyte. Habitat: Montane forest, 2000-2500 m. Vouchers: SAJIT 006813 (EA, HIB), FOKP 10913 \& 11707 (EA), Mbuni 707 (EA), Evans 811 (EA).

Huperzia ophioglossoides (Lam.) Rothm. Habit: Epiphyte. Habitat: Wet forest and montane forest, 1500-2500 m. Vouchers: SAJIT 007111 (EA, HIB), Burtt 1837 $\& 1838$ (EA).

Huperzia saururus (Lam.) Travis. Habit: Epiphyte. Habitat: Moorland, 25503500 m. Voucher: Mabberley \& McCall 185 (EA).

Lycopodium clavatum L. Habit: Herb. Habitat: Montane \& bamboo zone, 20002800 m. Vouchers: SAJIT 006821 (EA, HIB), Mabberley \& McCall 42 (EA).

\section{F2. Selaginellaceae}

1 Genus, 1 Species

Selaginella abyssinica Spring. Habit: Herb. Habitat: Often moist rock crevices, 1450-2300 m. Voucher: Agnew 5167 (EA). 
Part 2 Monilophytes

F3. Marattiaceae

1 Genus, 1 Species

Marattia fraxinea Sm. Habit: Herb. Habitat: Montane forest, 1500-2300 m. Voucher: FOKP 1119 (EA, HIB).

\section{F4. Hymenophyllaceae}

1 Genus, 1 Species

Crepidomanes melanotrichum (Schltdl.) J.P. Roux Habit: Epiphyte. Habitat: Wet forest on rocks, 1460-2570 m. Voucher: Thulin \& Tidigs 55 (EA).

\section{F5. Anemiaceae}

1 Genus, 1 Species

Mohria caffrorum (L.) Desv. Habit: Herb. Habitat: Rock crevices, 1800-2500 m. Voucher: Faden 824 (EA).

\section{F6. Cyatheaceae}

1 Genus, 1 Species

Cyathea manniana Hook. Habit: Tree. Habitat: Moist montane forest, in dense shade along streams, 1500-2500 m. Voucher: Mabberley 19 (EA).

\section{F7. Pteridaceae}

5 Genera, 10 Species

Adiantum poiretii Wikstr. Habit: Herb. Habitat: Dry montane forest, ericaceous zone, 100-3000 m. Vouchers: FOKP 1709 \& 1077 (EA, HIB).

Cheilanthes farinosa (Forssk.) Kaulf. Habit: Herb. Habitat: Bamboo and moorland zone, 1450-3000 m. Vouchers: SAJIT 004834 (EA, HIB), Mabberley \& McCall 240 (EA).

Cheilanthes bergiana Schltdl. Habit: Herb. Habitat: Moist shaded river bank, 16002300 m. Voucher: FOKP 1721 (EA, HIB).

Cheilanthes bergiana Schltdl. Habit: Herb. Habitat: Bamboo and moorland zone, 1450-3000 m. Voucher: FOKP 1721 (EA, HIB).

Cheilanthes quadripinnata Kuhn Habit: Herb. Habitat: Moist montane forest, bamboo zone, 1275-2750 m. Vouchers: FOKP 1090 (EA, HIB), Tweedie 3378 (EA).

Pteris catoptera Kunze Habit: Herb. Habitat: Forest swamp extending to bamboo zones, 1000-3050 m. Voucher: SAJIT 007091 (EA, HIB).

Pteris cretica L. Habit: Herb. Habitat: Dry montane forest, 1500-2700 m. Vouchers: SAJIT 004775 (EA, HIB), FOKP 1003 (EA, HIB), Thulin 56 (EA). 
Pteris dentata Forssk. Habit: Herb. Habitat: Upland forest, moist areas, 10003000 m. Vouchers: FOKP 1871 \& 11367 (EA, HIB), Mbuni 415 (EA).

Pteris vittata L. Habit: Herb. Habitat: Near streams in dense shade, 1250-1600 m. Voucher: Tweedie 2960 (EA).

Pteris pteridioides (Hook.) Ballard Habit: Herb. Habitat: Montane forest, 15502100 m. Voucher: FOKP 1726 (EA, HIB).

\section{F8. Aspleniaceae}

1 Genus, 10 Species

Asplenium abyssinicum Fée Habit: Herb. Habitat: Shade areas and bamboo zone, 1800-3500 m. Vouchers: Evans 812 (EA).

Asplenium aethiopicum (Burm. f.) Bech. Habit: Epiphyte. Habitat: Moist bushland, 1400-3600 m. Voucher: Part II Botany 221 (EA).

Asplenium bugoiense Hieron. Habit: Herb. Habitat: Moist montane forest, 20002700 m. Voucher: FOKP 1118 (EA, HIB).

Asplenium cordatum (Thunb.) Sw. Habit: Herb. Habitat: Moist, bush land, woodland, 1530-1830 m. Voucher: SAJIT Z0067 (EA, HIB).

Asplenium erectum Bory ex Willd. Habit: Herb. Habitat: Moist forest up to bamboo zone, 1300-2800 m. Voucher: FOKP 1122 (EA, HIB).

Asplenium linckii Kuhn Habit: Herb. Habitat: Moist forest up to bamboo zone, 1750-2500m. Voucher: FOKP 1896 (EA, HIB).

Asplenium loxoscaphoides Baker Habit: Herb. Habitat: Dense shade, 2000-2700m. Voucher: SAJIT 007117 (EA, HIB).

Asplenium monanthes L. Habit: Herb. Habitat: Bamboo zone, 2100-3400 m. Vouchers: FOKP 1078 (EA, HIB), Part II Botany 19 (EA).

Asplenium protensum Schrad. Habit: Epiphyte. Habitat: Moist montane forest, 1400-2900 m. Vouchers: SAJIT 004779 (EA, HIB), Part II Botany 18 (EA).

Asplenium theciferum (Kunth) Mett. Habit: Epiphyte. Habitat: Moist montane forest, 850-2900 m. Vouchers: FOKP 11642 (EA, HIB), Mbuni 642 (EA), Part II Botany 20 (EA).

F9. Athyriaceae

1 Genus, 1 Species

Deparia boryana (Willd.) M. Kato Habit: Herb. Habitat: Moist forest, 14602550 m. Voucher: FOKP 1893 (EA, HIB).

\section{F10. Dryopteridaceae}

4 Genera, 9 Species

Arachniodes webbiana (A. Braun) Schelpe. Habit: Herb. Habitat: Wet forest, 1550 2600 m. Voucher: SAJIT 007087 (EA, HIB). 
Dryopteris pentheri (Krasser) C. Chr. Habit: Herb. Habitat: Moist forest, 1550$2500 \mathrm{~m}$. Voucher: Tweedie 2681 (EA).

Dryopteris schimperiana (Hochst.) C. Chr. Habit: Herb. Habitat: Riverine forest, 2750-3220 m. Voucher: Tweedie 2844 (EA).

Elaphoglossum acrostichoides (Hook. \& Grev.) Schelpe Habit: Epiphyte. Habitat: Moist montane forest, 1750-2600 m. Voucher: Tweedie 2684 (EA).

Elaphoglossum deckenii (Kuhn) C. Chr Habit: Epiphyte. Habitat: Moist montane forest and bamboo zone, 2200-3300 m. Voucher: Beentje 3382 (EA).

Polystichum fuscopaleaceum Alston Habit: Herb. Habitat: Moist montane forest, along streams, 2100-2800 m. Voucher: Faden \& Evans 810 (EA).

Polystichum sinense (Christ) Christ. Habit: Herb. Habitat: Moist montane forest and Bamboo zone up to moorland, 2430-3500 m. Voucher: FOKP 1004 (EA, HIB).

Polystichum setiferum (Forssk.) Moore ex Woyn. Habit: Herb. Habitat: Moist montane forest, up to $3500 \mathrm{~m}$. Voucher: FOKP 11382 (EA, HIB).

Polystichum volkensii C. Chr. Habit: Herb. Habitat: Moist montane forest, up to 3300 m. Voucher: FOKP 1004 (EA, HIB).

\section{F2. Tectariaceae}

1 Genus, 1 Species

Tectaria gemmifera (Fée) Alston Habit: Herb. Habitat: Common in montane forest, 1200-2500 m. Voucher: Tweedie 2702 (EA).

\section{F12. Polypodiaceae}

4 Genera, 5 Species

Lepisorus excavatus (Bory ex Willd.) Ching Habit: Epiphyte. Habitat: Montane forest, ericaceous forest, 3150-3350 m. Vouchers: SAJIT 004784 \& 007056 (EA, HIB), Thulin \& Tidigs $99 \& 233$ (EA), FOKP 1743 (EA, HIB).

Drynaria volkensii Hieron. Habit: Epiphyte. Habitat: Moist montane forest, 1600$2300 \mathrm{~m}$. Voucher: Tweedie 2799 (EA).

Loxogramme abyssinica (Baker) M.G. Price Habit: Epiphyte. Habitat: Dry and wet forests, 1000-3000 m. Vouchers: FOKP 11701 (EA, HIB), Mbuni 701 (EA).

Pleopeltis excavata T. Moore Habit: Epiphyte. Habitat: Moist, riverine forest, 1000 3500 m. Voucher: FOKP 1743 (EA, HIB).

Pleopeltis macrocarpa (Bory ex Willd.) Kaulf. Habit: Epiphyte. Habitat: Moist forest, 1100-3550 m. Vouchers: Verdcourt et al. 2430 (EA), Beentje 3359 (EA). 
Part 3 Gymnosperms

\section{F13. Podocarpaceae}

2 Genera, 3 Species

Afrocarpus falcatus (Thunb.) C.N. Page. Habit: Tree. Habitat: Upland forest, 1250 2700 m. Voucher: SAJIT 007061 (EA, HIB).

Afrocarpus gracilior (Pilg.) C.N. Page Habit: Tree. Habitat: Upland forest, 15003300 m. Voucher: FOKP 1797 (EA, HIB).

Podocarpus latifolius (Thumb.) R. Br. ex Mirb. Habit: Tree. Habitat: Wet forest with bamboo, Hagenia, Juniperus and Olea forest, 1500-3300 m. Vouchers: FOKP $11611 \& 11770$ (EA, HIB), Mbuni 592 \& 611 (EA).

\section{F14. Cupressaceae}

1 Genus, 1 Species

Juniperus procera Hochst. ex Endl. Habit: Tree. Habitat: Drier upland forest, 10502950 m. Vouchers: FOKP 1799 \& 11272 (EA, HIB), Mbuni 08 (EA), Malombe \& Mlangeni 931 (EA).

\section{Part 4 Angiosperms}

\section{F15. Piperaceae}

2 Genera, 4 Species

Peperomia abyssinica Miq. Habit: Herb. Habitat: Upland forest, 1600-2950 m. Vouchers: FOKP 11389 \& 11547 (EA, HIB), Mbuni 284 (EA), Hepper \& Field 5034 (EA).

Peperomia retusa (L. f.) A. Dietr. Habit: Epiphyte. Habitat: Wet upland forest, 1500-2400 m. Vouchers: SAJIT 006889 (EA, HIB), FOKP 10989 (EA, HIB).

Peperomia tetraphylla (G. Forst.) Hook. \& Arn. Habit: Epiphyte. Habitat: Wet upland forest, 1400-2400 m. Vouchers: SAJIT $004816 \& 005065$ (EA, HIB), FOKP 1212 (EA, HIB).

Piper capense L. f. Habit: Herb. Habitat: Wet highland forest, 1200-2700 m. Voucher: FOKP 11416 (EA), Mbuni 152 (EA).

\section{F16. Annonaceae}

1 Genus, 2 Species

Uvaria leptocladon Oliv. Habit: Shrub. Habitat: Bushland, 0-1900 m. Vouchers: FOKP 11447 (EA), Mbuni 183 (EA).

Uvaria scheffleri Diels Habit: Liana. Habitat: Riverine, woodland, bushland, 9001800 m. Vouchers: FOKP 11469 (EA), Mbuni 205 (EA). 


\section{F17. Monimiaceae}

1 Genus, 1 Species

Xymalos monospora (Harv.) Baill. Habit: Tree. Habitat: Moist forest, 1400-2700 m. Vouchers: SAJIT 004857 (EA, HIB), FOKP 11362 \& 11372 (EA, HIB), Mbuni $108 \& 616$ (EA), Dale 912 (EA), Friis et al. 2514 (EA).

\section{F18. Lauraceae}

1 Genus, 1 Species

Ocotea kenyensis (Chiov.) Robyns \& R. Wilczek Habit: Tree. Habitat: Moist forest, 1700-2600 m. Voucher: FOKP 1808 (EA, HIB).

\section{F19. Araceae}

2 Genera, 3 Species

Arisaema enneaphyllum Hochst. ex A. Rich. Habit: Herb. Habitat: Montane forest edge, 2300-2900 m. Voucher: SAJIT 004859 (EA, HIB).

Arisaema mildbraedii Engl. Habit: Herb. Habitat: Wet montane forest edge, 1400 2620 m. Vouchers: SAJIT 006909 (EA, HIB), FOKP 11009 (EA, HIB).

Culcasia falcifolia Engl. Habit: Herb. Habitat: Marshy forest edges in high rain forest areas, 1370-2135 m. Vouchers: SAJIT 004753 (EA, HIB), FOKP 737 \& 1304 (EA, HIB).

\section{F20. Potamogetonaceae}

1 Genus, 1 Species

Potamogeton schweinfurthii A. Benn. Habit: Herb. Habitat: Artificial ponds, 18002000 m. Voucher: SAJIT 007071 (EA, HIB).

\section{F21. Dioscoreaceae}

1 Genus, 2 species

Dioscorea quartiniana A. Rich. Habit: Climber. Habitat: Wooded grassland and bushland, 0-2300 m. Vouchers: SAJIT 005067 (EA, HIB), FOKP 11627 \& 11765 (EA, HIB), Mbuni $587 \& 627$ (EA).

Dioscorea schimperiana Hochst. ex Kunth Habit: Climber. Habitat: Wooded grassland, 1600-2130 m. Vouchers: Waweru 32 (EA), Nappier 4150 (EA).

\section{F22. Colchicaceae}

3 Genera, 3 species

Gloriosa superba L. Habit: Herb. Habitat: Bushland, up to 2500 m. Vouchers: FOKP $11479 \& 11694$ (EA, HIB), Mbuni 215 \& 694 (EA). 
Androcymbium melanthioides Willd. Habit: Shrub. Habitat: Bushland, 1530-2680 m. Voucher: Blake 3090 (EA).

Wurmbea tenuis (Hook. f.) Baker Habit: Herb. Habitat: Grassland, 2130-2750 m. Voucher: Stewart 1002 (EA).

\section{F23. Smilacaceae}

1 Genus, 1 Species

Smilax anceps Willd Habit: Climber. Habitat: Wet forest, 10-2800 m. Voucher: FOKP 1071 (EA).

\section{F24. Orchidaceae}

23 Genera, 65 Species

Aerangis thomsonii (Rolfe) Schltr. Habit: Epiphyte. Habitat: Highland forest, on trunks of trees, above 2300 m. Vouchers: FOKP 11634 (EA), Mbuni 634 (EA).

Ancistrorhynchus clandestinus (Lindl.) Schltr. Habit: Epiphyte. Habitat: On tree branches, 1400-2500 m. Voucher: FOKP 11392 (EA, HIB).

Angraecum erectum Summerh. Habit: Epiphyte. Habitat: Drier forest, 1350-2000 m. Vouchers: FOKP 11303 \& 11305 (EA, HIB).

Angraecum thomsonii Rolfe Habit: Epiphyte. Habitat: Shady forest, 1800-2500 m. Voucher: SAJIT 007074 (EA, HIB).

Ansellia africana Lindl. Habit: Epiphyte. Habitat: Amongst tree roots, 0-2350 m. Vouchers: FOKP 11307 (EA, HIB), Mortimer 210 (EA), Webster 9023 (EA).

Brachycorythis ovata Lindl. Habit: Epiphyte. Habitat: Grassland, 2000-2550 m. Vouchers: Napier 1908 (EA), Maas 4691 (EA).

Bulbophyllum bequaertii De Wild. Habit: Epiphyte. Habitat: Shady forest, 17002350 m. Voucher: SAJIT 005098 (EA, HIB).

Bulbophyllum cochleatum Lindl. Habit: Epiphyte. Habitat: Shady forest, 14502000 m. Voucher: FOKP 11263 (EA, HIB).

Calanthe sylvatica (Thouars) Lindl. Habit: Herb. Habitat: Shady forest, 9002750 m. Voucher: Mabberley 486 (EA).

Cribbia brachyceras (Summerh.) Sengha Habit: Epiphyte. Habitat: Warm forest near river streams, 1700-2000 m. Voucher: SAJIT 006888 (EA, HIB).

Cynorkis anacamptoides Kraenzl. Habit: Herb. Habitat: Upland moorland and grassland, 2250-3350 m. Vouchers: Dale 3444 (EA), Thulin \& Tidigs 190 (EA).

Cynorkis kassneriana Kraenzl Habit: Epiphyte. Habitat: Upland rain forest, on mossy banks, 1600-2580 m. Voucher: Kirk 9418 (EA)

Diaphananthe rohrii (Rchb. f.) Summerh. Habit: Epiphyte. Habitat: Dry highland forest, above $2000 \mathrm{~m}$. Voucher: FOKP 11264 (EA, HIB).

Diaphananthe montana (Piers) P.J. Cribb \& J. Stewart Habit: Epiphyte. Habitat: Bushland, roots on moss and lichen, 2300-2750 m. Voucher: Stewart 1010 (EA). 
Diaphananthe pulchella Summerh. Habit: Epiphyte. Habitat: Dry forest, 15002330 m. Voucher: FOKP 11260 (EA, HIB).

Disa aconitoides subsp. goetzeana (Kraenzl.) H.P. Linder Habit: Herb. Habitat: Grassland, 2300-2550 m. Voucher: Webster 9036 (EA).

Disa fragrans subsp. deckenii (Rchb. f.) H.P. Linder Habit: Herb. Habitat: Grassland 2300-3350 m. Vouchers: Thulin \& Tidigs 205 (EA), Dale 3251 (EA).

Disa erubescens Rendle Habit: Herb. Habitat: Short upland grass, 1350-2550 m. Vouchers: Lucas 161 (EA), Blake 2128 (EA).

Disa concinna N.E. Br. Habit: Herb. Habitat: Grassland, 1450-2800 m. Voucher: Napier 1938 (EA).

Disa decknii Reichb. f. Habit: Herb. Habitat: Grassland, 2100-3300 m. Voucher: Dale 3251 (EA).

Disa hircicornis Rchb. f. Habit: Herb. Habitat: Wet grassland and swamps, often near streams, 1700-2750 m. Voucher: Dale 3446 (EA).

Disa stairsii Kraenzl. Habit: Herb. Habitat: Alpine grassland, Moorland and swamps, 2100-3550 m. Vouchers: Dale 3267 (EA), Thulin \& Tidigs 61 (EA).

Disperis kilimanjarica Rendle Habit: Herb. Habitat: Woodland, 2300-3000 m. Voucher: Mabberley \& McCall 13 (EA).

Disperis nemorosa Rendle Habit: Herb. Habitat: Mossy areas \& leaf litter, 18002850 m. Voucher: Dale 34431 (EA).

Epipactis africana Rendle Habit: Herb. Habitat: River banks, 2330-2830 m. Voucher: Mabberley 221 (EA).

Eulophia horsfallii (Bateman) Summerh. Habit: Herb. Habitat: Swamps and river edges, 1500-2700 m. Vouchers: Symes 525 (EA), Webster 9040 (EA).

Eulophia latilabris Summerh. Habit: Herb. Habitat: Swampy grassland, 15002200 m. Voucher: FOKP 11261 (EA, HIB).

Eulophia montis-elgonis Summerh. Habit: Herb. Habitat: Swampy grassland, 20002350 m. Vouchers: Blake 2016 (EA), Webster 9043 (EA).

Eulophia streptopetala Lindl. Habit: Herb. Habitat: Grassland, 1350-2330 m. Voucher: SAJIT 007076 (EA, HIB).

Eulophia taitensis Pfennig \& P.J. Cribb Habit: Herb. Habitat: Grassland, 10002500 m. Voucher: Archer 490 (EA).

Eulophia petersii (Rchb. f.) Rchb. f. Habit. Herb. Habitat: Grassland, Growing in sandy soil amongst Sansevieria, 0-2000 m. Vouchers: Tweedie $68 \& 139$ (EA).

Eulophia pyrophila (Rchb. f.) Summerh. Habit: Herb. Habitat: Grassland, 10002730 m. Voucher: Webster 9045 (EA).

Habenaria altior Rendle Habit: Herb. Habitat: Grassland, near streams, 18003500 m. Voucher: Dale 3447 (EA).

Habenaria cirrhata (Lindl.) Rchb. f. Habit: Herb. Habitat: Grassland, Scattered bushes, 300-1050 m. Voucher: Linday 157 (EA).

Habenaria clavata (Lindl.) Rchb. f. Habit: Herb. Habitat: Grassland, 1000-2730 m. Vouchers: Jex-Blake 2127 (EA), Agnew et al. 10580 (EA). 
Habenaria malacophylla Rchb. f. Habit: Herb. Habitat: Upland rain, grassland forest, 1350-2700 m. Vouchers: SAJIT 005091 (EA, HIB), FOKP 11645 (EA, HIB), Mbuni 645 (EA), Part II Botany 43 (EA).

Habenaria walleri Rchb. f. Habit: Herb. Habitat: Swampy grassland, 1200-2300 m. Voucher: Webster 9027 (EA).

Habenaria macruroides Summerh. Habit: Herb. Habitat: Open bushland, 18502200 m. Voucher: Tweedie 456 (EA).

Habenaria holubii Rolfe Habit: Herb. Habitat: Swamp grassland, 1200-2300 m. Voucher: Blake 2127 (EA).

Holothrix elgonensis Summerh. Habit: Herb. Habitat: Grassland, 3300-3500 m. Voucher: FOKP 11313 (EA, HIB).

Holothrix brongniartiana Rchb. f. Habit: Herb. Habitat: Grassland, 2300-3200 m. Vouchers: Daicin 3442 (EA), Thulin \& Tidigs 150 (EA).

Holothrix pentadactyla (Summerh.) Summerh. Habit: Herb. Habitat: Upland grassland, open rocky ground, 2500-3500 m. Vouchers: Bally 5706 (EA), Townsend 2345(EA), Jex-Blake 5706 (EA), Sharpe 5706 (EA), Stewart 1005 (EA).

Liparis deistelii Schltr. Habit: Herb. Habitat: Mossy banks, tree trunks, 20002700 m. Vouchers: SAJIT 005094 (EA, HIB), Stewart 1099 (EA).

Polystachya bella Summerh. Habit: Epiphyte. Habitat: High up in forest trees, 1800-2300 m. Vouchers: FOKP 11420 (EA), Mbuni 156 (EA).

Polystachya bennettiana Rchb. f. Habit: Epiphyte. Habitat: Drier forest, 16502330 m. Vouchers: Webster $1981 \& 9030$ (EA).

Polystachya bicarinata Rendle Habit: Epiphyte. Habitat: On river streams, 23502830 m. Vouchers: SAJIT 004826 \& 005099 (EA, HIB), FOKP $11259 \& 1012$ (EA, HIB), Van Someren 8650 (EA).

Polystachya campyloglossa Rolfe Habit: Epiphyte. Habitat: Highland forest, 17003000 m. Voucher: FOKP 11263 (EA, HIB).

Polystachya cultriformis (Thouars) Lindl. ex Spreng. Habit: Epiphyte. Habitat: River banks, 330-2700 m. Vouchers: SAJIT 005095 (EA, HIB), Webster 9029 (EA). Polystachya eurychila Summerh. Habit: Epiphyte. Habitat: On rocks, 1830-2300 m. Voucher: Kirk \& Irwin 50 (EA).

Polystachya spatella Kraenzl. Habit: Epiphyte. Habitat: Highland forest, 2000-2830 m. Vouchers: FOKP 11646 (EA), Mbuni 646 (EA), Friis \& Hansen 2510 (EA).

Polystachya stricta Rolfe Habit: Epiphyte. Habitat: Highland forest, 2000-2830 m. Voucher: Webster 9030 (EA).

Polystachya tenuissima Kraenzl. Habit: Epiphyte. Habitat: Highland forests, 1700 2500 m. Vouchers: SAJIT 005096 (EA, HIB), Tweedie 449 (EA).

Roeperocharis bennettiana Rchb. f. Habit: Epiphyte. Habitat: Swamps, 23002800 m. Voucher: Dale 3445 (EA).

Satyrium carsonii Rolfe Habit: Herb. Habitat: Open grassland, 2000-2330 m, Elgeyo open grassland. Vouchers: Napier 2126 (EA), Jex-Blake 2126 (EA).

Satyrium coriophoroides A. Rich. Habit: Herb. Habitat: Grassland, 1700-2800 m. Vouchers: Symes 95 (EA), Tweedie 1589 (EA). 
Satyrium crassicaule Rendle Habit: Herb. Habitat: Swamps, 1850-3300 m. Voucher: Webster 9034 (EA).

Satyrium fimbriatum Summerh. Habit: Herb. Habitat: Grassland, 2000-3300 m. Vouchers: Stewart 1000 (EA), Dale 3269 (EA), Lucas 163 (EA).

Satyrium sceptrum Schltr. Habit: Herb. Habitat: Grassland, 1700-2800 m. Voucher: Thulin 144 (EA).

Satyrium schimperi Hochst. ex A. Rich. Habit: Herb. Habitat: Grassland, 2300 3200 m. Vouchers: Irwin 426 (EA), Mainwaring 61 (EA).

Satyrium yunnanense Rolfe Habit: Herb. Habitat: Grassland, 1700-2800 m. Voucher: FOKP 11265 (EA, HIB).

Stolzia repens (Rolfe) Summerh. Habit: Epiphyte. Habitat: Riverine forest, 19502900 m. Voucher: FOKP 1094 (EA).

Tridactyle anthomaniaca (Rchb. f.) Summerh. Habit: Epiphyte. Habitat: Warm forest, above $1650 \mathrm{~m}$. Voucher: SAJIT 004791 (EA, HIB).

Tridactyle furcistripes Summerh. Habit: Epiphyte. Habitat: highland forest, 23003100 m. Vouchers: Bally 2538 (EA), Tweedie 2173 (EA), Beentje 3055 (EA).

Tridactyle nigrescens Summerh. Habit: Epiphyte. Habitat: Riverine, Woodland, 1150-2200 m. Voucher: Jackson \& Tweedie 3492 (EA).

Tridactyle scottellii (Rendle) Schltr. Habit: Epiphyte. Habitat: Rocky grassland and highland forest, 2230-3000 m. Voucher: Beentje 3050 (EA).

\section{F25. Hypoxidaceae}

I Genus, 5 species

Hypoxis angustifolia Lam. Habit: Herb. Habitat: Grassland in upland, 1300-3000 m. Vouchers: Napier 1907 (EA), Symes 44 \& 286 (EA).

Hypoxis kilimanjarica Baker Habit: Herb. Habitat: Grassland, 2900-3250 m. Voucher: Thulin \& Tidigs 201 (EA).

Hypoxis obtusa Burch. ex Ker Gawl. Habit: Herb. Habitat: Burnt grassland, 18002500 m. Vouchers: Agnew 10484 (EA), Napier 1942 (EA), Symes 287 (EA), Webster 9022 (EA).

Hypoxis urceolata Nel Habit: Herb. Habitat: Grassland, 1300-3000 m. Voucher: Symes 51 (EA).

Hypoxis goetzei Harms Habit: Herb. Habitat: Burnt grassland, 1830-1920 m. Voucher: Symes 285 (EA).

\section{F26. Iridaceae}

7 Genera, 14 Species

Aristea abyssinica Pax. Habit: Herb. Habitat: Wet grassland, 1700-3500 m. Voucher: Tweedie 2985 (EA).

Aristea abyssinica Pax Habit: Herb. Habitat: Grassland, 2200-2850 m. Voucher: Blake 3108 (EA). 
Aristea cognata N.E. Br. ex Weim. Habit: Herb. Habitat: Grassland, 2200-2850 m. Voucher: Symes 155 (EA).

Dierama cupuliflorum Klatt Habit: Herb. Habitat: Grassland, 2400-3500 m. Voucher: Thulin \& Tidings 207 (EA).

Dierama pendulum (L. f.) Baker Habit: Herb. Habitat: Grassland, 2400-3500 m. Voucher: Lucas 216 (EA).

Freesia laxa (Thunb.) Goldblatt \& J.C. Manning Habit: Herb. Habitat: Rocky grassland, $1700-3500 \mathrm{~m}$. Voucher: Lucas 188 (EA).

Gladiolus dalenii Van Geel Habit: Herb. Habitat: Damp upland grassland, 12003100 m. Voucher: Maas 4690 (EA).

Gladiolus dichrous (Bullock) Goldblatt Habit: Herb. Habitat: Stony highland grassland, 2592-3050 m. Voucher: Mabberley \& MacCall 151 (EA).

Gladiolus schweinfurthii (Baker) Goldblatt \& M.P. de Vos Habit: Herb. Habitat: Montane grassland, 1830-2290 m. Voucher: Dale 3449 (EA).

Gladiolus watsonioides Baker. Habit: Herb. Habitat: Bamboo and alpine zone, 2400-3550 m. Voucher: FOKP 1812 (EA, HIB).

Hesperantha petitiana (A. Rich.) Baker Habit: Herb. Habitat: Subalpine and alpine grassland, 1520-3500 m. Voucher: Thulin \& Tidings 216 (EA).

Morea thomsonii Baker Habit: Herb. Habitat: Stony grassland, 1400-1920 m. Voucher: Symes 296 (EA).

Romulea camerooniana Baker Habit: Herb. Habitat: Subalpine, 1520-3500 m. Voucher: Maas 6040 (EA).

Romulea fischeri Pax Habit: Herb. Habitat: Wet upland, 1520-3500 m. Voucher: Thulin \& Tidings 110 (EA), Dowson 619 (EA).

\section{F27. Xanthorrhoeaceae}

2 Genera, 5 species

Aloe cheranganiensis S. Carter \& Brandham Habit: Shrub. Habitat: Dry scrub, bushed grassland when open, 1200-1900 m. Voucher: Brandham 1727 (EA).

Aloe dawei A. Berger Habit: Shrub. Habitat: Rocky bushland, 1830-2120 m. Voucher: FOKP 11326 (EA, HIB).

Aloe penduliflora Baker Habit: Shrub. Habitat: Rocky ground, 2100-2500 m. Vouchers: FOKP 11675 (EA), Mbuni 675 (EA).

Aloe secundiflora Engl. Habit: Shrub. Habitat: Sandy soils in drier areas, 900-1800 m. Vouchers: Spoerry 2 \& 14 (EA).

Kniphofia thomsonii Baker Habit: Herb. Habitat: Highland grassland, 1900-3550 m. Vouchers: SAJIT 005116 (EA, HIB), Mabberley \& MacCall 251 (EA), Lucas 212 (EA).

\section{F28. Amaryllidaceae}

2 Genera, 2 Species

Allium neapolitanum Cirillo Habit: Herb. Habitat: Upland grassland, 1500-2500 m. Vouchers: FOKP 11299 (EA, HIB), Mbuni 035 (EA, HIB). 
Scadoxus multiflorus (Martyn) Raf. Habit: Herb. Habitat: Rocky places in forest edges, riverine forest, 5-2250 m. Voucher: SAJIT 007081 (EA, HIB).

\section{F29. Asparagaceae}

5 Genera, 10 Species

Anthericum angustifolium Hochst. ex A. Rich. Habit: Herb. Habitat: Grassland, 1980-2450 m. Voucher: Blake 1896 (EA).

Asparagus africanus Lam. Habit: Shrub. Habitat: Bushy wooded, 100-2680 m. Voucher: Symes 281 (EA).

Asparagus falcatus L. Habit: Climber. Habitat: Upland forest, Woodland, 102750 m. Vouchers: FOKP 11699 (EA), Mbuni 699 (EA).

Asparagus racemosus Willd. Habit: Climber. Habitat: Drier bushland 1160-2800 m. Vouchers: FOKP 954, 1018, 11288 \& 11699 (EA, HIB), Mbuni 024 \& 699 (EA), Webster 9000 (EA), Mus 114 (EA).

Chlorophytum cameronii (Baker) Kativu Habit: Herb. Habitat: Highland grassland, 1675-2450 m. Voucher: Symes 97 (EA).

Chlorophytum cameronii var. pterocaulon (Welw. ex Baker) Nordal Habit: Herb. Habitat: Highland grassland, 1675-2450 m. Vouchers: FOKP 11689 (EA), Mbuni 689 (EA), Symes 36 (EA).

Chlorophytum affine Baker Habit: Herb. Habitat: Dry bushland, 15-1980 m. Vouchers: Symes 609 (EA), Napier \& Tweedie 2119 (EA).

Dracaena ellenbeckiana Engl. Habit: Tree. Habitat: Bushland, 1050-1950 m. Voucher: SAJIT 005050 (EA, HIB).

Dracaena afromontana Mildbr. Habit: Tree. Habitat: Bamboo forest, 1900-2400 m. Vouchers: FOKP 1070, 11398 \& 11522 (EA), Mbuni 134 \& 258 (EA), Oteke 97 (EA).

Ornithogalum gracillimum R.E. Fr. Habit: Herb. Habitat: Grassland, 1800-2700 m. Voucher: SAJIT 005076 (EA, HIB).

\section{F30. Commelinaceae}

5 Genera, 11 Species

Aneilema recurvatum Faden Habit: Herb. Habitat: Grassland, 700-1300 m. Voucher: Faden 78 (EA).

Aneilema sebitense Faden Habit: Herb. Habitat: Woodland, 1500-1550 m. Vouchers: Faden 803A (EA), Mabberley \& McCall 88 \& 88A (EA).

Aneilema zebrinum Chiov. ex Chiarugi. Habit: Herb. Habitat: Woodland, 101150 m. Voucher: Faden et al. 888 (EA).

Cyanotis barbata D. Don Habit: Herb. Habitat: Grassland, 1650-3200 m. Vouchers: Stewart 1008 (EA), Symes 617 (EA).

Commelina africana var. krebsiana (Kunth) C.B. Clarke Habit: Herb. Habitat: Grassland, 700-2300 m. Voucher: Lye 907 (EA).

Commelina benghalensis L. Habit: Herb. Habitat: Grassland, bushland, disturbed habitats, 10-2200 m. Vouchers: FOKP 11415 (EA), Mbuni 151 (EA). 
Commelina foliacea Chiov. Habit: Herb. Habitat: Grassland, 350-1550 m. Vouchers: Faden 807 (EA), Mabberley \& McCall 88 (EA).

Commelina imberbis Ehrenb. ex Hassk. Habit: Herb. Habitat: Cultivated ground, disturbed habitat, 1000-1750 m. Voucher: Faden 805 (EA).

Commelina triangulispatha Mildbr. Habit: Herb. Habitat: Moist roadside banks, 1650-2300 m. Vouchers: Tweedie 4083 (EA), Faden \& Evans 487 (EA).

Floscopa glomerata (Willd. ex Schult. \& Schult. f.) Hassk. Habit: Herb. Habitat: Standing water in forest, 1650-2200 m. Vouchers: Ivens 1251 (EA), Webster 8995 (EA).

Murdannia edulis (Stokes) Faden Habit: Herb. Habitat: Grassland, bushland, 9001300 m. Voucher: Maas 6373 (EA).

\section{F31. Eriocaulaceae}

1 Genus, 3 Species

Eriocaulon abyssinicum Hochst. Habit: Herb. Habitat: Wet shallow soils, 16502730 m. Voucher: SAJIT 005160 (EA, HIB).

Eriocaulon inyangense Arw. Habit: Herb. Habitat: Swamps in grassland, around $900 \mathrm{~m}$. Voucher: Townsend 1435 (EA).

Eriocaulon schimperi Körn. ex Ruhland Habit: Herb. Habitat: Heath zones, 24403100 m. Vouchers: SAJIT 006822 (EA, HIB), FOKP 945, 1133, 1243 \& 10922, (EA, HIB), Fries \& Hansen 2533 (EA), Rauh 678 (EA).

\section{F32. Juncaceae}

2 Genera, 5 Species

Juncus dregeanus Kunth Habit: Herb. Habitat: Upland and alpine forest, 29003300 m. Vouchers: FOKP 1148 (EA, HIB), Bogdan 4991 (EA).

Juncus effusus L. Habit: Herb. Habitat: Highland and moorland forest, 1500-3300 m. Voucher: FOKP 1151 (EA, HIB).

Juncus oxycarpus E. Mey. ex Kunth Habit: Herb. Habitat: Upland streamsides and alpine seepage zones, 1500-2750 m. Vouchers: FOKP 1241 (EA, HIB), Verdcourt 2435 (EA).

Luzula abyssinica Parl. Habit: Herb. Habitat: High forest streamsides and wet alpine, 2000-3500 m. Voucher: Bogdan 10100 (EA).

Luzula johnstonii Buchenau Habit: Herb. Habitat: Alpine wet grassland, 24003500 m. Vouchers: Thulin \& Tidigs 244 (EA), Mabberley 193 (EA).

\section{F33. Cyperaceae}

9 Genera, 40 Species

Bulbostylis densa subsp. afromontana (Lye) R.W. Haines Habit: Herb. Habitat: Shallow soils, 1500-3000 m. Voucher: Knight 71 (EA).

Carex bequaertii De Wild. Habit: Herb. Habitat: Alpine grassland and montane swamps, 2300-3500 m. Voucher: Thulin \& Tidigs 49 (EA). 
Carex chlorosaccus C.B. Clarke Habit: Herb. Habitat: Swampy edges, 1500-3300 m. Vouchers: Lye 9125 (EA), Gehrke \& Muasya 165 (EA).

Carex erythrorrhiza Boeckeler Habit: Herb. Habitat: Streams, 2500-3500 m. Voucher: Kirk 9887 (EA).

Carex elgonensis Nelmes Habit: Herb. Habitat: Alpine swamps, 1500-3300 m. Voucher: Part II Botany 75 (EA).

Carex johnstonii Boeckeler Habit: Herb. Habitat: High forest edges, 2200-3300 m. Voucher: Thulin \& Tidigs 48 (EA).

Carex lycurus K. Schum. ex Engl. Habit: Herb. Habitat: Montane river-sides, 1950$3300 \mathrm{~m}$. Voucher: Thulin 50 (EA).

Carex mannii E.A. Bruce Habit: Herb. Habitat: Montane forest grades, 22003300 m. Voucher: Gehrke \& Muasya 167 (EA).

Carex monostachya A. Rich. Habit: Herb. Habitat: Disturbed bushland, alpine openings, above 2700 m. Voucher: Kirk 58 (EA).

Carex peregrina Link Habit: Herb. Habitat: Common in alpine around, $2300 \mathrm{~m}$. Voucher: Thulin \& Tidigs 112 (EA).

Carex petitiana A. Rich. Habit: Herb. Habitat: Montane forest grades, 2500-3500 m. Voucher: Beentje 212 (EA).

Carex steudneri Boeckeler Habit: Herb. Habitat: Grassland \& woodland edges, 2300-3000 m. Voucher: Bogdan 4972 (EA).

Carex simensis Hochst. ex A. Rich. Habit: Herb. Habitat: Alpine grassland, 19003500 m. Voucher: Thulin 243 (EA).

Cyperus aterrimus Hochst. ex Steud. Habit: Herb. Habitat: Alpine grassland, 19003500 m. Voucher: Ivens 1246 (EA).

Cyperus alternifolius L. Habit: Herb. Habitat: Montane forest, 2200-3300 m. Vouchers: Agnew et al. 10458 (EA), Mus 244 (EA), Mbuni 683 (EA).

Cyperus cyperoides (L.) Kuntze Habit: Herb. Habitat: Montane forest, 1600-3500 m. Voucher: Hanson \& Thomasson 57 (EA).

Cyperus distans L. Habit: Herb. Habitat: Temporary water, marshes and ferns up to 2500 m. Voucher: Ivens 1247 (EA).

Cyperus derreilema Steud. Habit: Herb. Habitat: Upland forest and streamsides, 2000-3000 m. Voucher: Ivens 1245 (EA).

Cyperus dichrostachyus Hochst. ex A. Rich. Habit: Herb. Habitat: Highland swamps, 1200-2400 m. Vouchers: SAJIT 004745 (EA, HIB), FOKP 997 \& 1152 (EA, HIB).

Cyperus involucratus Rottb. Habit: Herb. Habitat: Upland forest, 1800-3000 m. Voucher: Lugard 2793 (EA).

Cyperus impubes Steud. Habit: Herb. Habitat: Forest shade, 1400-2500 m. Voucher: Maas 4701 (EA).

Cyperus iria L. Habit: Herb. Habitat: Disturbed wet forests, 1000-1500 m. Voucher: Bogdan 5169 (EA).

Cyperus kerstenii Boeckeler Habit: Herb. Habitat: Alpine, Moorland 2500-3500 m. Voucher: Smith et al, 194 (EA).

Cyperus mollipes (C.B. Clarke) K. Schum. Habit: Herb. Habitat: Grassland, 100 2750 m. Voucher: Bogdan 4528 (EA). 
Cyperus cruentus Rottb. Habit: Herb. Habitat: Grassland, 500-2000 m. Voucher: Leippert 5116 (EA).

Cyperus nutans var. eleusinoides (Kunth) Haines Habit: Herb. Habitat: streamside, swampy areas, 1000-2200 m. Voucher: Bogdan 5308 (EA).

Cyperus platycaulis Baker Habit: Herb. Habitat: Wet areas, 1000-3000 m. Vouchers: FOKP 980 \& 1134 (EA, HIB), Smith, Beentje \& Muasya 208 (EA), Botany 2 Exp. 74 (EA).

Cyperus rotundus L. Habit: Herb. Habitat: Grassland, Up to 2000 m. Vouchers: Mbuni 014 (EA), FOKP 11278 (EA).

Cyperus schimperianus Steud. Habit: Herb. Habitat: River-side swamps, Sandy, stony river banks, grassland, 450-1600 m. Voucher: Thomas 2117 (EA).

Cyperus squarrosus L. Habit: Herb. Habitat: Wet pool edges, up to $2000 \mathrm{~m}$. Voucher: Leippert 5041 (EA).

Fimbristylis dichotoma (L.) Vahl Habit: Herb. Habitat: Grassland, up to $2000 \mathrm{~m}$. Voucher: Bogdan 4960 (EA).

Fuirena stricta subsp. chlorocarpa (Ridl.) Lye Habit: Herb. Habitat: Wet grassland, 500-2800 m. Voucher: Bogdan 4992 (EA).

Isolepis costata Hochst. ex A. Rich. Habit: Herb. Habitat: Alpine, Sedge in dense growth, 1700-3500 m. Voucher: Bogdan 4990 (EA).

Isolepis fluitans (L.) R. Br. Habit: Herb. Habitat: Montane water, 1300-3500 m. Voucher: Thulin \& Tidigs 97 (EA).

Kyllinga alba Nees Habit: Herb. Habitat: Dry bushland, 500-2500 m. Voucher: Napper \& Tweedie 2124 (EA).

Kyllinga brevifolia Rottb Habit: Herb. Habitat: Streamside, 1600-3000 m. Vouchers: FOKP 11731 (EA), Mbuni 731 (EA).

Kyllinga odorata Vahl Habit: Herb. Habitat: Streamside, 1300-3300 m. Vouchers: Smith et al. 196 (EA), Bogdan 5002 (EA).

Pycreus elegantulus (Steud.) C.B. Clarke Habit: Herb. Habitat: Grassland, 10002200 m. Vouchers: FOKP 990 \& 1130 (EA, HIB).

Kyllinga pulchella Kunth Habit: Herb. Habitat: Woodland edge, 1500-2600 m. Voucher: Smith et al. 190 (EA).

Schoenoplectus corymbosus (Roth ex Roem. \& Schult.) J. Raynal Habit: Herb. Habitat: Grassland, 1000-2500 m. Vouchers: FOKP 984, 1141 (EA, HIB) Bogdan 5313 (EA), Muasya 1284 (EA).

\section{F34. Poaceae}

40 Genera, 59 Species

Agrostis kilimandscharica Mez. Habit: Herb. Habitat: Bamboo and upland, 20003500 m. Voucher: Bogdan 4974 (EA).

Agrostis producta Pilg. Habit: Herb. Habitat: Upland grassland \& moorland 25003500 m, Vouchers: Bogdan 4977 (EA), Thulin et al. 256 (EA). 
Agrostis quinqueseta (Steud.) Hochst. Habit: Herb. Habitat: Moorland \& alpine, 3000-3500 m. Voucher: Bogdan 4973 (EA).

Aira caryophyllea L. Habit: Herb. Habitat: Grassland and moorland above $2000 \mathrm{~m}$. Vouchers: Bogdan 4989 (EA), Braun 582 (EA).

Andropogon amethystinus Steud. Habit: Herb. Habitat: Alpine grassland, 20003550 m. Vouchers: Thulin \& Tidigs 225 (EA), Braun 581 (EA).

Andropogon chinensis (Nees) Merr. Habit: Herb. Habitat: Savannah and bushland, 0-1800 m. Voucher: Bogdan 3829 (EA).

Andropogon schirensis Hochst. Habit: Herb. Habitat: Grassland, 2000-3500 m. Voucher: Strange 82 (EA).

Anthephora nigritana Stapf \& C.E. Hubb. Habit: Herb. Habitat: Rocky bushland, 1200-1700 m. Vouchers: Bogdan 3407 \& 5633 (EA).

Anthoxanthum nivale K. Schum. Habit: Herb. Habitat: Upland grassland \& moorland, above 2500 m. Vouchers: Bogdan 4971 \& Bogdan 4997 (EA).

Aristida adscensionis L. Habit: Herb. Habitat: Bushland, 1500-2500 m. Voucher: Bogdan 5632 (EA).

Aristida hordeacea Kunth Habit: Herb. Habitat: Bushland, 400-1600 m. Voucher: Bogdan 3414 (EA).

Bothriochloa radicans (Lehm.) A. Camus Habit: Herb. Habitat: Deciduous bushland, 0-2000 m. Voucher: Bogdan 3413 (EA).

Brachiaria scalaris Pilg. Habit: Herb. Habitat: Upland weedy places, 900-2400 m. Vouchers: Bogdan 5306 (EA), Baner 481 (EA).

Brachypodium flexum Nees Habit: Herb. Habitat: Upland forest, 2000-3000 m. Voucher: Bogdan 4870 (EA).

Cymbopogon giganteus Chiov. Habit: Herb. Habitat: Bushland, 0-2300 m. Voucher: Bogdan 3854 (EA).

Digitaria gayana (Kunth) A. Chev. Habit: Herb. Habitat: Disturbed place, farmland, 1200-2000 m. Voucher: Bogdan 4337 (EA).

Digitaria velutina (Forssk.) P. Beauv. Habit: Herb. Habitat: Disturbed places, 0-2300 m. Voucher: Baner 480 (EA).

Dinebra retroflexa (Vahl) Panz. Habit: Herb. Habitat: Black clay soils, 500-2000 m. Voucher: Pratt 5716 (EA).

Ebrharta erecta Lam. Habit: Herb. Habitat: Forest clearing, 1500-2700 m. Voucher: Bogdan 5305 (EA).

Eragrostis cylindriflora Hochst. Habit: Herb. Habitat: Dry bushland, 1200-2000 m. Voucher: Bogdan 3422 (EA).

Eragrostis patens Oliv. Habit: Herb. Habitat: Dry land area, 400-2000 m. Voucher: Bogdan 3423 (EA).

Eragrostis tremula Hochst. ex Steud. Habit: Herb. Habitat: Grassland, 0-1600 m. Voucher: Bogdan 4492 (EA).

Exotheca abyssinica (Hochst. ex A. Rich.) Andersson Habit: Herb. Habitat: Upland and montane grassland, 2000-3550 m. Voucher: Strange 85 (EA). 
Festuca abyssinica A. Rich. Habit: Herb. Habitat: Highland grassland and moorland, 2000-3000 m. Voucher: Heady 1415 (EA).

Hyparrhenia pilgeriana C.E. Hubb. Habit: Herb. Habitat: Swamps, 1000-2700 m. Voucher: Bogdan 4513 (EA).

Hyparrhenia variabilis Stapf. Habit: Herb. Habitat: Deciduous bushland, wooded grassland, 600-2200 m. Voucher: Bogdan 3635 (EA).

Koeleria capensis Nees Habit: Herb. Habitat: Upland Grassland and moorland, 2000-3550 m. Voucher: Thulin \& Tidigs 249 (EA).

Leersia hexandra Sw. Habit: Herb. Habitat: Shallow water and swamps, up to 2000 m. Voucher: Ivens 1283 (EA).

Leptochloa rupestris C.E. Hubb. Habit: Herb. Habitat: Deciduous bushland, rocky places, 1600-1900 m. Voucher: Bogdan 3844 (EA).

Loudetia flavida (Stapf) C.E. Hubb. Habit: Herb. Habitat: Dry bushland, 3001600 m. Voucher: Deg 32 (EA).

Loudetia kagerensis (K. Schum.) C.E. Hubb. Habit: Herb. Habitat: Stony soil, 1200-2700 m. Voucher: Kuchar 8653 (EA).

Microchloa indica (L. f.) P. Beauv. Habit: Herb. Habitat: Low altitude, shallow soil, 800-1600 m. Voucher: Bogdan 5171 (EA).

Melinis repens (Willd.) Zizka Habit: Herb. Habitat: Grassland, 0-2500 m. Voucher: FOKP 1034 (EA).

Melinis nerviglumis (Franch.) Zizka Habit: Herb. Habitat: Upland grassland and open woodland, 500-2000 m. Voucher: Bogdan 3426 (EA).

Oplismenus compositus (L.) P. Beauv. Habit: Herb. Habitat: Evergreen grassland, 0-2400 m. Voucher: Bogdan 4952 (EA).

Oplismenus hirtellus (L.) P. Beauv. Habit: Herb. Habitat: Forest shade, 0-2500 m. Voucher: Bogdan 4952 (EA).

Oplismenus undulatifolius (Ard.) Roem. \& Schult. Habit: Herb. Habitat: Forest shade, 1400-2500 m. Voucher: Mabberley \& McCall 60 (EA).

Oropetium minimum (Hochst.) Pilg. Habit: Herb. Habitat: Open deciduous, dry grassland, 780-2600 m. Voucher: Bogdan 4948 (EA).

Oropetium thomaeum (L. f.) Trin. Habit: Herb. Habitat: Deciduous grassland, 500 1100 m. Voucher: Bogdan 4949 (EA).

Panicum atrosanguineum Hochst. ex A. Rich. Habit: Herb. Habitat: Dry bushland, 100-2200 m. Voucher: Baner 482 (EA).

Panicum pusillum Hook. f. Habit: Herb. Habitat: Grassland, moorland, Streams, 1300-3300 m. Voucher: Bogdan 5299 (EA).

Panicum hochstetteri Steud. Habit: Herb. Habitat: Drier in forest shade, 15003000 m. Voucher: Bogdan 3835 (EA).

Panicum hymeniochilum Nees. Habit: Herb. Habitat: Wet places, 1000-3000 m. Vouchers: Thulin \& Tidigs 180, Thulin et al. 161 (EA).

Pennisetum macrourum Trin. Habit: Herb. Habitat: River banks \& Streams, 7002300 m. Voucher: Bogdan 3637 (EA). 
Pennisetum sphacelatum (Nees) T. Durand \& Schinz. Habit: Herb. Habitat: Bushland, 1500-3200 m. Voucher: Thulin \& Tidigs 277 (EA).

Pennisetum squamulatum Fresen. Habit: Herb. Habitat: Deciduous bushland, 3001600 m. Voucher: Bogdan 3833 (EA).

Pogonarthria squarrosa (Roem. \& Schult.) Pilg. Habit: Herb. Habitat: Open moorland, 500-2000 m. Voucher: Bogdan 3447 (EA).

Pogonarthria squarrosa (Roem. \& Schult.) Pilg. Habit: Herb. Habitat: Sandy grassland and open woodland, 500-1870 m. Voucher: Bogdan 3447 (EA).

Polypogon schimperianus (Hochst. ex Steud.) Cope Habit: Herb. Habitat: Moorland \& alpine, 3000-3500 m. Voucher: Ivens 1244 (EA).

Sacciolepis africana C.E. Hubb. \& Snowden. Habit: Herb. Habitat: Shallow water up to $1600 \mathrm{~m}$. Voucher: Knight 5309 (EA).

Sacciolepis indica (L.) Chase Habit: Herb. Habitat: streamside marshes, 10002200 m. Voucher: Bogdan 5309 (EA).

Schmidtia pappophoroides Steud. ex J.A. Schmidt Habit: Herb. Habitat: Bushland, 200-2000 m. Voucher: Bogdan 3845 (EA).

Setaria orthosticha K. Schum. ex R.A.W. Herrm. Habit: Herb. Habitat: Evergreen grassland, 1000-2300 m. Voucher: Bogdan 3851 (EA).

Sporobolus panicoides A. Rich. Habit: Herb. Habitat: Deciduous bushland, 11002000 m. Voucher: Bogdan 3884 (EA).

Stipa keniensis (Pilg.) Freitag Habit: Herb. Habitat: Upland forest shade and road sides, 2000-2700 m. Voucher: Bogdan 500 (EA).

Streblochaete longiarista (A. Rich.) Pilg. Habit: Herb. Habitat: forest shade \& bamboo clearing, 1500-3000 m. Voucher: Thorold 2758 (EA).

Urochloa oligotricha (Fig. \& De Not.) Henrard. Habit: Herb. Habitat: Wooded grassland, 1300-2000 m. Voucher: Bogdan 3409 (EA).

Vulpia bromoides (L.) Gray Habit: Herb. Habitat: Rocky soils, 2500-3500 m. Vouchers: Verdcourt 2439A (EA), Taylor et al. 2439 (EA).

Yushania alpina (K. Schum.) W.C. Lin Habit: Herb. Habitat: Montane forest 30003300 m. Voucher: Davidse 9224 (EA).

\section{F35. Papaveraceae}

1 Genus, 1 Species

Corydalis mildbraedii Fedde Habit: Herb. Habitat: Grassland, 2200-3500 m. Voucher: Thulin et al. 235 (EA).

\section{F36. Menispermaceae}

2 Genera, 2 Species

Cissampelos pareira L. Habit: Herb. Habitat: Woodland, 2-2600 m. Vouchers: FOKP 11289 \& 11604 (EA, HIB), Mbuni 593 \& 604 (EA). 
Stephania abyssinica (Quart.-Dill. \& A. Rich.) Walp. Habit: Liana. Habitat: bushland, 1950-2600 m. Vouchers: SAJIT 005058 (EA, HIB), FOKP 934 (EA, HIB).

\section{F37. Berberidaceae}

1 Genus, 1 Species

Berberis holstii Engl. Habit: Shrub. Habitat: Upland bushland, 2150-3000 m. Voucher: SAJIT 007059 (EA, HIB).

\section{F38. Ranunculaceae}

6 Genera, 9 Species

Anemone thomsonii Oliv. Habit: Herb. Habitat: Grassland, 2500-3500 m. Vouchers: Bogdan 4994 (EA), Knox 3392 (EA).

Clematis brachiata Thunb. Habit: Climber. Habitat: Wooded grassland, 720 3150 m. Vouchers: FOKP 11277 (EA, HIB), Webster 8708 (EA), Lind 5094 (EA), Ivens 1254 (EA).

Clematis simensis Fresen. Habit: Climber. Habitat: Grassland 1600-3250 m. Vouchers: FOKP 993, $1022 \& 11512$ (EA, HIB), Mbuni 248 (EA), Lind 2851 (EA), Knox 3857 (EA), Mabberley et al. 227 (EA).

Clematis villosa DC. Habit: Herb. Habitat: Grassland, 1600-3250 m Vouchers: Symes 42 (EA), Webster 8710 (EA).

Delphinium macrocentrum Oliv. Habit: Herb. Habitat: grassland, 1650-3500 m. Vouchers: FOKP 11387 (EA, HIB), Lind 5099 (EA), Mabberley 235 (EA).

Ranunculus multifidus Forssk. Habit: Herb. Habitat: streamside, 1350-3450 m. Vouchers: FOKP 1137, 1207 \& 11360 (EA, HIB), Mbuni 096 (EA), Mabberley $\&$ McCall 213 (EA), Thulin et al. 78 (EA).

Ranunculus oreophytus Delile Habit: Herb. Habitat: streamside, 2550-3500 m. Vouchers: Knox 3383 (EA), Trelawny 4385 (EA).

Ranunculus volkensii Engl. Habit: Herb. Habitat: Grassland, 2550-3400 m. Vouchers: Thulin \& Tidigs 194 (EA), Knox 3382 (EA).

Thalictrum rhynchocarpum Dillon \& A. Rich Habit: Herb. Habitat: Upland forest, 1550-3275 m. Vouchers: SAJIT 005057 (EA, HIB), FOKP 11533 (EA), Mbuni 269 (EA), Mabberley \& McCall 188 (EA).

\section{F39. Proteaceae}

2 Genera, 6 Species

Faurea arborea Engl. Habit: Tree. Habitat: Wooded grassland, 1600-2400 m. Voucher: Thulin \& Tidings 181 (EA).

Faurea rochetiana (A. Rich.) Chiov. ex Pic. Serm. Habit: Tree. Habitat: Wooded grassland, 1600-2400 m. Vouchers: FOKP 11325 (EA, HIB), Birch 61 \& 178 (EA). 
Faurea saligna Harv. Habit: Tree. Habitat: Wooded grassland, 1600-2400 m. Vouchers: SAJIT 004766 (EA, HIB), FOKP 1092 \& 11268 (EA, HIB), Mbuni 004 (EA), Trapnell 2301 (EA), Botany Part II 61 (EA), Buch 178 (EA).

Protea caffra subsp. kilimandscharica (Engl.) Chisumpa \& Brummitt Habit: Shrub. Habitat: Moorland, 2500-3500 m. Voucher: Dale 862 (EA).

Protea gaguedi J.F. Gmel. Habit: Tree. Habitat: Grassland bushland, 1500-2900 m. Vouchers: SAJIT 005146 (EA, HIB), FOKP 11275 (EA), Mbuni 014 (EA), Thulin \& Tidigs 73 (EA), Napper 1504 (EA).

Protea madiensis Oliv. Habit: Shrub. Habitat: Wooded grassland, 1650-1900 m. Voucher: Buch 161 (EA).

\section{F40. Gunneraceae}

1 Genus, 1 Species

Gunnera perpensa L. Habit: Herb. Habitat: River-side marshes, 2300-3370 m. Voucher: Tweedie 3014 (EA).

\section{F41. Crassulaceae}

5 Genera, 16 Species

Bryophyllum pinnatum (Lam.) Oken Habit: Herb. Habitat: Grassland, 10002100 m. Voucher: Symes 2799 (EA).

Crassula alata (Viv.) A. Berger Habit: Herb. Habitat: Grassland, 1350-2700 m. Voucher: Maas 6348 (EA).

Crassula alba Forssk. Habit: Herb. Habitat: Grassland, 2439-3048 m. Vouchers: SAJIT 005115 \& 004811 (EA, HIB), Dale 3271 (EA).

Crassula alsinoides (Hook. f.) Engl. Habit: Herb. Habitat: Wet rocky, 1475-3500 m. Vouchers: SAJIT 006816 (EA, HIB), FOKP 10916 \& 11530 (EA, HIB), Mbuni 266 (EA), Mabberley 572 (EA).

Crassula granvikii Mildbr. Habit: Herb. Habitat: Alpine 1650-3500 m. Vouchers: FOKP 1214 \& 11733 (EA, HIB), Mbuni 112 \& 733 (EA), Thulin 204 (EA).

Crassula schimperi Fisch. \& C.A. Mey. Habit: Herb. Habitat: Upland grassland, 1050-3550 m. Voucher: SAJIT 004833 (EA).

Crassula vaginata Eckl. \& Zeyh. Habit: Herb. Habitat: Upland grassland, 20003500 m. Voucher: Lucas 214 (EA).

Kalanchoe citrina Schweinf. Habit: Herb. Habitat: Bushland, 1150-2100 m. Voucher: Tweedie 1773 (EA).

Kalanchoe crenata (Andrews) Haw. Habit: Herb. Habitat: Grassland, 1650-2300 m. Vouchers: SAJIT 006894 (EA, HIB), Symes 279 (EA).

Kalanchoe densiflora Rolfe Habit: Herb; Habitat: Montane grassland, 1500-3000 m. Vouchers: SAJIT 006793 (EA, HIB), FOKP 11380 (EA), Mbuni 129 (EA), Thulin \& Tidigs 85 (EA), Rauh 683 (EA). 
Kalanchoe glaucescens Britten Habit: Herb. Habitat: Bushland, 900-2200 m. Vouchers: FOKP 1279 (EA, HIB), Mortimer 207 (EA).

Kalanchoe lanceolata (Forssk.) Pers. Habit: Herb. Habitat: Grassland, Dry areas, 950-2100 m. Vouchers: SAJIT 006899 (EA, HIB), Tweedie 1774 (EA).

Kalanchoe prittwitzii Engl. Habit: Herb. Habitat: Bushland, 1650-2300 m. Voucher: SAJIT 006845 (EA, HIB).

Sedum meyeri-johannis Engl. Habit: Herb. Habitat: Heartland, 2100-3150 m. Vouchers: SAJIT 006824 (EA, HIB), FOKP 10924 (EA, HIB).

Sedum ruwenzoriense Baker f. Habit: Herb. Habitat: Upland heartland, 28003500 m. Vouchers: Mabberley \& McCall 230 (EA), Symes 649 (EA).

Umbilicus botryoides Hochst. ex A. Rich. Habit: Herb. Habitat: Montane forest, 2250-3500 m. Voucher: SAJIT 004850 (EA).

\section{F42. Haloragaceae}

1 Genus, 1 Species

Laurembergia tetrandra (Schott) Kanitz Habit: Herb. Habitat: Wet grassland at streamside, 2000-2350 m. Vouchers: SAJIT 006823 (EA, HIB), FOKP 10923 (EA, HIB).

\section{F43. Vitaceae}

4 Genera, 14 Species

Ampelocissus africana (Lour.) Merr. Habit: Liana. Habitat: Riverine, woodland, 1450-1800 m. Voucher: Napier 2000 (EA).

Cissus petiolata Hook. f. Habit: Liana. Habitat: Riverine forest, 1050-1800 m. Voucher: Balley 12355 (EA).

Cissus rotundifolia Vahl Habit: Shrub. Habitat: Acacia-Commiphora bushland, 0-2100 m. Voucher: SAJIT Z0041 (EA, HIB).

Cissus ruspolii Gilg Habit: Climber. Habitat: Bushland, 1300-1620 m. Voucher: Meyerhoff 102 (EA).

Cyphostemma bambuseti (Gilg \& M. Brandt) Desc. ex Wild \& R.B. Drumm. Habit: Climber. Habitat: Dry upland forest, 1450-2400 m. Voucher: SAJIT 004750 (EA, HIB).

Cyphostemma junceum (Baker) Desc. ex Wild \& R.B. Drumm. Habit: Climber. Habitat: Grassland 1650-2280 m. Voucher: Symes 292 (EA).

Cyphostemma cyphopetalum (Fresen.) Desc. ex Wild \& R.B. Drumm. Habit: Climber. Habitat: Bushland, 470-2450 m. Vouchers: FOKP 11752 (EA), Mbuni 752 (EA), Symes 334 (EA).

Cyphostemma dysocarpum (Gilg \& M. Brandt) Desc. Habit: Climber. Habitat: Acacia-Commiphora bushland, 1000-1600 m. Voucher: Lindsay 129 (EA).

Cyphostemma heterotrichum (Gilg \& R.E. Fr.) Desc. ex Wild \& R.B. Drumm. Habit: Climber. Habitat: Grassland, 1600-2300 m. Vouchers: Napier 2028 (EA), Symes 103 (EA). 
Cyphostemma kilimandscharicum (Gilg) Desc. ex Wild \& R.B. Drumm. Habit: Climber. Habitat: Montane rain forest, 1600-3040 m. Voucher: SAJIT 005070 (EA, HIB). Cyphostemma maranguense (Gilg) Desc. Habit: Climber. Habitat: Dry upland forest, 1600-3040 m. Voucher: FOKP 11276 (EA, HIB).

Cyphostemma pseudosesquipedale Verdc. Habit: Herb. Habitat: Grassland up to 1372 m. Voucher: Agnew et al. 10230 (EA).

Cyphostemma Serpens (Hochst. ex A. Rich.) Desc. Habit: Climber. Habitat: Bushed grassland, 1200-2550 m. Vouchers: SAJIT 006876 (EA, HIB), FOKP 11281 (EA, HIB).

Rhoicissus tridentata (L. f.) Wild \& R.B. Drumm. Habit: Shrub. Habitat: Wooded, 60-2700 m. Vouchers: SAJIT 004846 (EA, HIB), FOKP 1108, 1262, 11280 \& 11451 (EA, HIB), Mbuni $187 \& 679$ (EA).

\section{F44. Zygophyllaceae}

1 Genus, 1 Species

Tribulus terrestris L. Habit: Herb. Habitat: Waste places and trodden earth, 102300 m. Vouchers: FOKP 11488 (EA), Mbuni 224 (EA).

\section{F45. Fabaceae}

39 Genera, 98 Species

Vachellia abyssinica Benth. Habit: Tree. Habitat: Wooded grassland, 1450-3300 m. Vouchers: FOKP 11269 (EA), Mbuni 005 (EA).

Senegalia brevispica Harms Habit: Tree. Habitat: Dry bushland, thickets, 1701830 m. Vouchers: SAJIT Z0058 (EA, HIB), Napier 2037 (EA).

Vachellia hockii De Wild. Habit: Tree. Habitat: Wooded grassland, 750-2250 m. Vouchers: FOKP 1266 (EA, HIB), Mbuni 654 (EA).

Vachellia labai Benth. Habit: Tree. Habitat: Upland forest, dense woodland, 15002700 m. Vouchers: Mbuni 005 (EA), Napier 1952 \& 2014 (EA), Padwa 56 (EA), Brown 577 (EA).

Mimosa oerfota (Forssk.) Schweinf. Habit: Tree. Habitat: Riverine woodland, 15002900 m. Voucher: Sooboda 58 (EA).

Racosperma mearnsii De Wild. Habit: Tree. Habitat: Wooded grassland, 9002600 m. Vouchers: FOKP 11561 (EA, HIB), Mbuni 298 (EA).

Mimosa mellifera (M. Vahl) Benth. Habit: Tree. Habitat: Bushland grassland, Dry woodland, 1-1800 m. Vouchers: SAJIT Z0064 (EA, HIB), FOKP 1252 (EA, HIB).

Mimosa nilotica (L.) Delile Habit: Tree. Habitat: Woodland grassland, 1-2300 m. Vouchers: Mus 169 (EA), Bogdan 4340 (EA), Collias 15382 (EA).

Vachellia tortilis subsp. spirocarpa (A. Rich.) Brenan Habit: Tree. Habitat: Bushland along streams, 200-1650 m. Voucher: FOKP 1249 (EA, HIB).

Vachellia reficiens Wawra Habit: Tree. Habitat: Bushland, 50-1450 m. Voucher: FOKP 1257 (EA, HIB). 
Adenocarpus mannii (Hook. f.) Hook. f. Habit: Shrub. Habitat: Shrub Grassland, riverside, hearth, bamboo zone, 2400-3500 m. Voucher: SAJIT 007113 (EA, HIB).

Albizia anthelmintica Brongn. Habit: Tree. Habitat: Dry bushland, 1-3550 m. Voucher: Kazuaki 52 (EA).

Argyrolobium fischeri Taub. Habit: Herb. Habitat: Bushland, grassland, 17002700 m. Vouchers: SAJIT 005102 (EA, HIB), FOKP 1805 (EA, HIB).

Argyrolobium ramosissimum Baker. Habit: Herb. Habitat: Forest margin, moorland, ericaceous, around $2300 \mathrm{~m}$. Voucher: Rawlins 7 (EA).

Astragalus atropilosulus (Hochst.) Bunge. Habit: Herb. Habitat: Open moorland, 1750-3550 m. Voucher: SAJIT 005084 (EA, HIB).

Caesalpinia decapetala (Roth) Alston Habit: Shrub. Habitat: Bushland, Forest edges, 650-2050 m. Voucher: FOKP 1297 (EA, HIB).

Cassia abbreviata Oliv. Habit: Shrub. Habitat: Bushland, 1-1000 m. Voucher: SAJIT 004734 (EA, HIB).

Crotalaria anthyllopsis Baker Habit: Herb. Habitat: Wooded grassland, 12002250 m. Rocky places. Voucher: Tweedie 1921 (EA).

Crotalaria axillaris Aiton Habit: Shrub. Habitat: Secondary bushland, woodland, grassland 1-2250 m. Vouchers: FOKP 1870, 11414 \& 11613 (EA, HIB), Mbuni 150 (EA), Part II Botany 13 (EA).

Crotalaria brevidens Benth. Habit: Shrub. Habitat: Grassland, bushland, 15003000 m. Vouchers: FOKP 11482 (EA), Mbuni 218 (EA), Napier 1979 (EA).

Crotalaria chrysochlora Harms Habit: Herb. Habitat: Upland grassland, 17002900 m. Vouchers: Napier 1916 (EA), Webster 8786 (EA).

Crotalaria deserticola Baker f. Habit: Shrub. Habitat: Open and wooded grassland, 100-2500 m. Vouchers: FOKP 11483 (EA, HIB), Mbuni 219 (EA).

Crotalaria fascicularis Polhill Habit: Shrub. Habitat: Dry evergreen forest, 13002300 m. Voucher: Champion 1 (EA).

Crotalaria incana L. Habit: Shrub. Habitat: Upland grassland, 600-1800 m. Vouchers: FOKP 11347 (EA, HIB), Mbuni 083 (EA).

Crotalaria lachnocarpoides Engl. Habit: Shrub. Habitat: Bushed Grassland, forest thicket, forest margin, 1200-2650 m. Vouchers: FOKP 11573 (EA, HIB), Mbuni 310 (EA).

Crotalaria microcarpa Benth. Habit: Herb. Habitat: Bushland, Grassland, 1001700 m. Voucher: Tweedie 2040 (EA).

Crotalaria natalitia Meissner Habit: Herb. Habitat: Forest edges, bushland and wooded or grassland, 1300-2500 m. Voucher: Symes 116 (EA).

Crotalaria pallida var. obovata (G. Don) Polhill. Habit: Herb. Habitat: Bushland, near rivers and lakes, 450-1800 m. Voucher: Bogdan 4963 (EA).

Crotalaria pallida Aiton Habit: Herb. Habitat: Bushland near rivers, 1100-1900 m. Voucher: Tweedie 2097 (EA).

Crotalaria podocarpa DC Habit: Herb. Habitat: Open sandy place, wooded grassland, 1300-1650 m. Voucher: Bogdan 5173 (EA). 
Crotalaria stolzii (Baker f.) Polhill Habit: Herb. Habitat: Upland grassland, forest margin, 1800-3000 m. Voucher: Thulin \& Tidigs 163 (EA).

Dalbergia lactea Vatke Habit: Shrub. Habitat: Riverine, 1050-2400 m. Vouchers: SAJIT 006880 (EA, HIB), FOKP 1289 (EA, HIB), Tweedie 1084 (EA).

Desmodium incanum DC. Habit: Herb. Habitat: Forest margin, 1450-2800 m. Vouchers: FOKP 11527 (EA, HIB), Mbuni 263 (EA).

Desmodium uncinatum (Jacq.) DC. Habit: Shrub. Habitat: Forest shade, 14002800 m. Vouchers: (FOKP 11715 EA, HIB), Mbuni 715 (EA).

Dinebra retroflexa (Vahl) Panz. Habit: Herb. Habitat: Black clay soil, 500-2000 m. Voucher: Pratt 5716 (EA).

Dolichos sericeus subsp. formosus (A. Rich.) Verdc. Habit: Climber. Habitat: Evergreen montane forest edge, 1150-2750 m. Vouchers: SAJIT 00686 (EA, HIB), Tweedie 2959 (EA).

Dolichos sericeus E. Mey. Habit: Herb. Habitat: Montane forest edge, 1150-2750 m. Voucher: Irwin 369 (EA).

Entada abyssinica A. Rich. Habit: Tree. Habitat: Wooded grassland, Riverine forest, 1450 2150 m. Vouchers: SAJIT 004842 \& 006851 (EA, HIB), FOKP 1277 (EA, HIB).

Entada leptostachya Harms. Habit: Climber. Habitat: Dry bushland, 100-1350 m. Voucher: SAJIT 004842 (EA, HIB).

Eriosema buchananii Baker f. Habit: Herb. Habitat: upland grassland, 19002300 m. Voucher: Napier 1971 (EA).

Eriosema cordifolium A. Rich. Habit: Herb. Habitat: Upland grassland about 2290 m. Voucher: Symes 526 (EA).

Eriosema macrostipulum Baker f. Habit: Herb. Habitat: Bushed grassland around 2100 m. Voucher: Symes 61 (EA).

Eriosema montanum Baker f. Habit: Herb. Habitat: Wooded grassland, 15003300 m. Voucher: Symes 203 (EA).

Eriosema rhodesicum R.E. Fr. Habit: Herb. Habitat: Burnt upland grassland, rocky hill side, 1620-2400 m. Voucher: Symes 277 (EA).

Erythrina abyssinica DC. Habit: Tree. Habitat: Open woodland, forest edge, 9002250 m. Vouchers: FOKP 11488 (EA, HIB), Mbuni 224 (EA), Trapnell 2302 (EA).

Faidherbia albida (Delile) A. Chev. Habit: Tree. Habitat: Wooded grassland, 15002900 m. Voucher: Tweedie 2288 (EA).

Galega lindblomii (Harms) J.B. Gillett. Habit: Herb. Habitat: Mountain grassland, 2200-2800 m. Vouchers: SAJIT Z0071, FOKP 949 \& 1143, Geesteranus 6320 (EA), Gillett 18423 (EA).

Glycine wrightii Lopez. Habit: Climber. Habitat: Disturbed ground, 2000-2900 m. Vouchers: FOKP 11450 (EA, HIB), Mbuni186 (EA).

Hylodesmum repandum (Vahl) H. Ohashi \& R.R. Mill Habit: Herb. Habitat: Forest shade, medium altitude forest, 1450-2800 m. Vouchers: FOKP 11525 (EA, HIB), Mbuni 261 (EA).

Indigofera ambelacensis Schweinf. Habit: Herb. Habitat: Grassland and bushland, 1000-2200 m. Vouchers: FOKP 11475 (EA, HIB), Mbuni 211 (EA). 
Indigofera arrecta A. Rich. Habit: Herb. Habitat: Grassland and bushland and forest edges, 300-2700 m. Vouchers: FOKP 11497 \& 11714 (EA, HIB), Mbuni 233 \& 714 (EA), Bogdan 5358 (EA).

Indigofera astragalina DC. Habit: Herb. Habitat: Grazed grassland, 600-900 m. Vouchers: Bogdan 3410 (EA), Tweedie 2894 (EA).

Indigofera bogdanii J.B. Gillett Habit: Shrub. Habitat: Dry grassland, 1500-2300 m. Voucher: Gillet 18423 (EA).

Indigofera garckeana Vatke Habit: Herb. Habitat: Grassland and bushland, 10002500 m. Vouchers: Agnew et al. 10388 (EA), Irwin 144 (EA).

Indigofera hochstetteri Baker. Habit: Herb. Habitat: Dry grassland, 200-2000 m. Voucher: Bogdan 3842 (EA).

Indigofera schimperi Jaub. \& Spach. Habit: Herb. Habitat: Grassland and bushland, 200-2200 m. Vouchers: FOKP 11747 (EA, HIB), Mbuni 747 (EA).

Indigofera spicata Forssk. Habit: Herb. Habitat: Grassland, 0-2900 m. Vouchers: FOKP 11502 (EA, HIB), Mbuni 238 (EA).

Indigofera trita L. f. Habit: Herb. Habitat: Grassland and bushland, 0-2500 m Vouchers: SAJIT 004747 (EA, HIB), FOKP 1288 (EA, HIB).

Lablab purpureus (L.) Sweet. Habit: Climber. Habitat: Riverine forest, 1-2400 m. Voucher: Webster 8788 (EA).

Lonchocarpus eriocalyx Harms Habit: Tree. Habitat: Wooded grassland, 5001680 m. Voucher: Bally 12334 (EA).

Lotus becquetii Boutique. Habit: Herb. Habitat: Rocky hill side, 2000-2800 m. Voucher: Strange 185 (EA).

Macrotyloma stipulosum (Baker) Verdc. Habit: Herb. Habitat: Wooded grassland, 1800-2150 m. Voucher: Symes 59 (EA).

Neonotonia wightii (Wight \& Arn.) J.A. Lackey Habit: Tree. Habitat: Bushland edges, 500-2100 m. Voucher: FOKP 1283 (EA, HIB).

Neorautanenia mitis (A. Rich.) Verdc. Habit: Climber. Habitat: Dry bushland, 201800 m. Voucher: Padwa 291 (EA).

Parochetus communis D. Don. Habit: Herb. Habitat: Moist area, 2200-3400 m. Voucher: FOKP 1908 \& 1904 (EA, HIB).

Pseudarthria confertiflora (A. Rich.) Baker Habit: Shrub. Habitat: Wooded grassland, 1-2200 m. Voucher: Webster 8793 (EA).

Pterolobium stellatum (Forssk.) Brenan Habit: Shrub. Habitat: Bushland, Woodland, 1000-2250 m. Voucher: FOKP 1265 (EA, HIB).

Rhynchosia hirta (Andrews) Meikle \& Verdc. Habit: Liana. Habitat: Dry woodland and bushland, 1-1800 m. Voucher: SAJIT 006841 (EA, HIB).

Rhynchosia orthobotrya Harms. Habit: Herb. Habitat: Dry rocky, bushland, 10002100 m. Voucher: Bogdan 3852 (EA).

Rhynchosia resinosa (A. Rich.) Baker. Habit: Liana. Habitat: Wooded grassland, bushland, 1530-2500 m. Voucher: Tweedie 1914 (EA).

Rhynchosia usambarensis Taub. Habit: Herb. Habitat: Upland grassland forest edge, 1650-2400 m. Voucher: FOKP 1284 (EA, HIB). 
Senna bicapsularis (L.) Roxb. Habit: Shrub. Habitat: Wooded or bushed grassland, 1-1750 m. Voucher: Kuzuaki 9 (EA).

Senna didymobotrya (Fresen.) H.S. Irwin \& Barneby Habit: Shrub. Habitat: Riverine, forest edge, 1500-2250 m. Vouchers: FOKP 11391 (EA, HIB), Mbuni 126 (EA).

Senna hirsuta (L.) H.S. Irwin \& Barneby. Habit: Shrub. Habitat: Bushed grassland 100-1750 m. Vouchers: FOKP 11422 (EA), Mbuni 158 (EA).

Senna obtusifolia (L.) H.S. Irwin \& Barneby. Habit: Shrub. Habitat: Riverine, 1000-1750 m. Voucher: Brodhurst hill 677 (EA).

Senna occidentalis (L.) Link. Habit: Shrub. Habitat: Cultivated ground, 2501450 m. Vouchers: FOKP 11487 (EA), Mbuni 223 (EA), Ndegwa 974 (EA).

Senna septemtrionalis (Viv.) H.S. Irwin \& Barneby Habit: Shrub. Habitat: Riverine, grassland, waste places, 1450-2200 m. Voucher: FOKP 1903 (EA, HIB).

Sesbania sesban (L.) Merr. Habit: Shrub. Habitat: Water logged areas below $1900 \mathrm{~m}$. Vouchers: FOKP 11493 (EA, HIB), Mbuni 229 (EA).

Tamarindus indica L. Habit: Tree. Habitat: Riverine, bushland, 1-1500 m. Vouchers: Tweedie 65 (EA), Kazuaki 16 (EA).

Tephrosia holstii Taub. Habit: Herb. Habitat: Upland grassland, 1600-2600 m. Voucher: Symes 101 (EA).

Tephrosia interrupta Engl. Habit: Herb. Habitat: Scrub margin, 1400-3100 m. Vouchers: FOKP 11289 (EA), Mbuni 025 (EA), Thulin 120 (EA).

Tephrosia vogelii Hook. f. Habit: Herb. Habitat: Waste ground up to $2300 \mathrm{~m}$. Voucher: Hepper 5058) (EA)

Teramnus labialis (L. f.) Spreng. Habit: Climber. Habitat: Wooded grassland, 17002500 m. Voucher: Boonman 6627 (EA).

Vigna subterranea (L.) Verdc. Habit: Herb. Habitat: Cultivated land below $1200 \mathrm{~m}$. Voucher: FOKP 1716 (EA, HIB).

Trifolium burchellianum Ser. Habit: Herb. Habitat: Moist upland, 1600-3500 m. Vouchers: FOKP 951 (EA, HIB), Mabberley \& McCall 242 (EA), Bogdan 4966 (EA).

Trifolium cheranganiense J.B. Gillett Habit: Herb. Habitat: Upland grassland, 2100-3100 m, Vouchers: Thorold 2759 (EA), Strange 48 (EA), Rawlins 2 (EA).

Trifolium cryptopodium A. Rich. Habit: Herb. Habitat: Grassland, 2100-3500 m. Vouchers: Thulin \& Tidigs 113 (EA), Bogdan 4968 (EA).

Trifolium polystachyum Fresen. Habit: Herb. Habitat: Forest margin, Swamp grassland, 1600-2800 m. Vouchers: Irwin 308 (EA), Symes 637 (EA).

Trifolium rueppellianum Fresen. Habit: Herb. Habitat: Upland grassland and moorland, 1700-3500 m. Vouchers: FOKP 1128 (EA, HIB), Strange 140 (EA).

Trifolium simense Fresen. Habit: Herb. Habitat: Upland grassland, 2000-3500 m. Vouchers: Symes 638 (EA), Knight 56 (EA).

Trifolium semipilosum Fresen. Habit: Herb. Habitat: Upland grassland, 15003000 m. Vouchers: FOKP 11501 (EA), Mbuni 237 (EA).

Trifolium usambarense Taub. Habit: Herb. Habitat: Forest opening, 1500-2700 m. Voucher: SAJIT Z0074 (HIB). 
Vicia benghalensis L. Habit: Climber. Habitat: Upland grassland 2400-2800 m. Voucher: Irwin 205 (EA).

Vigna monophylla Taub. Habit: Herb. Habitat: Wooded grassland, 1650-2500 m. Voucher: Symes 86 (EA).

Vigna unguiculata subsp. dekindtiana (Harms) Verdc. Habit: Herb. Habitat: Bushland and forest edges, 1-2500 m. Voucher: Lyes 57 (EA).

Vigna vexillata (L.) A. Rich. Habit: Herb. Habitat: Grassland, bushland and forest margins, 100-2500 m. Voucher: Symes 212 (EA).

Zornia glochidiata DC. Habit: Herb. Habitat: Grassland, rocky areas, 0-1800 m. Voucher: Bogdan 3450 (EA).

\section{F46. Polygalaceae}

1 Genus 4 Species

Polygala albida Schinz Habit: Herb. Habitat: Field of Weeds and roadside, 14002300 m. Voucher: Symes 758 (EA).

Polygala arenaria Willd. Habit: Herb. Habitat: Disturbed ground, 60-1200 m. Voucher: Lucas 218 (EA).

Polygala petitiana A. Rich. Habit: Herb. Habitat: Shallow soils on grassland, 1002300 m. Voucher: Symes 158 (EA).

Polygala sphenoptera Fresen. Habit: Herb. Habitat: Upland grassland, disturbed soil on wet forest, 100-3200 m. Vouchers: SAJIT $005100 \&$ Z0051 (EA, HIB), Mbuni 087 (EA), Carter \& Stannard 48 (EA), Mabberley \& McCall 279 (EA), Symes 217 (EA), Webster 8717 (EA).

\section{F47. Rosaceae}

5 Genera, 15 Species

Alchemilla cryptantha Steud. ex A. Rich. Habit: Herb. Habitat: Montane grassland 2900-3500 m. Vouchers: Napier 1978 (EA), Knox 4185 \& 4193 (EA).

Alchemilla elgonensis Mildbr. Habit: Shrub. Habitat: Montane grassland, 30003500 m. Voucher: Thulin \& Tidigs 248 (EA).

Alchemilla ellenbeckii Engl. Habit: Shrub. Habitat: Highland grassland, 21003500 m. Voucher: Knox 3384 (EA).

Alchemilla gracilipes (Engl.) Engl. Habit: Herb. Habitat: Montane grassland 21203500 m. Vouchers: SAJIT 006928 (EA, HIB), Dale 3400 (EA), Symes 619 (EA), Verdcourt 2426 (EA).

Alchemilla johnstonii Oliv. Habit: Shrub. Habitat: Alpine forest, 3150-3350 m. Vouchers: Dale 340, Thulin \& Tidigs 212 \& 258 (EA), Knox 3385 (EA).

Alchemilla lindblomiana (Mildbr.) T.C.E. Fr. Habit: Shrub. Habitat: 3000-3300 m.

Voucher: Dale 3401(EA) 
Alchemilla rothii Oliv. Habit: Herb. Habitat: Bamboo zones, 2100-3500 m. Vouchers: Mabberley 579 (EA), Knox 3404 (EA).

Cliffortia nitidula (Engl.) R.E. Fr. \& T.C.E. Fr. Habit: Shrub. Habitat: Bamboo glades, moorland, 2920-3490 m. Voucher: Dale 3432 (EA).

Hagenia abyssinica (Bruce ex Steud.) J.F. Gmel. Habit: Tree. Habitat: Woodland, 2300-3300 m. Voucher: SAJIT 004854 (EA, HIB).

Prunus africana (Hook. f.) Kalkman Habit: Tree. Habitat: Woodland, Riverine, 1350-2750 m. Vouchers: SAJIT 006869 (EA, HIB), FOKP 1019, 10969 \& 11609 (EA, HIB), Mbuni 609 (EA).

Rubus apetalus Poir. Habit: Shrub. Habitat: Upland bushland, 1600-3000 m. Vouchers: FOKP 1060 \& 11274 (EA, HIB).

Rubus pinnatus Willd. Habit: Trailing Shrub. Habitat: Upland rain forest, bamboo 2400-3000 m. Voucher: Tweedie 4086 (EA).

Rubus scheffleri Engl. Habit: Shrub. Habitat: Upland rain forest glades, 1800-2930 m. Vouchers: Mabberley 496 (EA), Lindsay 137 (EA).

Rubus steudneri Schweinf. Habit: Shrub. Habitat: Montane forest, 2000-3480 m. Vouchers: SAJIT 003385, $005121 \& 006811$ (EA, HIB), FOKP 1010 \& 10911 (EA, HIB), Beentje 3065 (EA), Tweedie 4086 (EA).

Rubus volkensii Engl. Habit: Shrub. Habitat: Secondary bushland, bamboo zones, 2600-3500 m. Vouchers: SAJIT 004800 (EA, HIB), Thulin \& Tidigs 242 (EA).

\section{F48. Rhamnaceae}

6 Genera, 8 Species

Gouania longispicata Engl. Habit: Shrub. Habitat: Wooded grassland, 1450-2400 m. Vouchers: SAJIT 005057 (EA, HIB), FOKP 955, 1030, 2335 \& 11278 (EA, HIB).

Helinus integrifolius (Lam.) Kuntze Habit: Shrub. Habitat: Bushland, open woodland, 300-1330 m. Voucher: FOKP 1098 (EA, HIB).

Helinus mystacinus (Aiton) E. Mey. ex Steud. Habit: Shrub. Habitat: 1000-1500 m. Voucher: FOKP 1098 (EA, HIB).

Rhamnus prinoides L'Hér. Habit: Tree. Habitat: Bushland, Bamboo, heath zone, 1500-3150 m. Vouchers: SAJIT 004817 \& 005080 (EA, HIB), FOKP 1001, 1226, $11310 \& 11366$, (EA, HIB), Mbuni 046 (EA).

Rhamnus staddo A. Rich. Habit: Shrub. Habitat: Bushland, Grassland, 15002950 m. Vouchers: FOKP 11310 \& 11652 (EA, HIB), Mbuni 046 \& 652 (EA).

Scutia myrtina (Burm. f.) Kurz Habit: Shrub. Habitat: Moist, dry forest, riverine up to 2750 m. Vouchers: SAJIT 005064 (EA, HIB), FOKP 943, 11266, 11309 \& 11378 (EA, HIB), Mbuni 045 (EA).

Ziziphus abyssinica Hochst. ex A. Rich. Habit: Tree. Habitat: Bushland, woodland, 700-2200 m. Voucher: FOKP 11318 (EA, HIB).

Ziziphus mucronata Willd. Habit: Tree. Habitat: Bushland, Wooded Grassland, 1-1950 m. Voucher: FOKP 11334 (EA, HIB). 


\section{F49. Ulmaceae}

1 Genus, 1 Species

Chaetachme aristata Planch. Habit: Tree. Habitat: Bushland, 1050-2100 m. Voucher: FORK 927 (EA, HIB).

\section{F50. Cannabaceae}

2 Genera, 2 Species

Celtis africana Burm. f. Habit: Tree. Habitat: Riverine forest, 1150-2400 m. Voucher: Verdcourt 11725 (EA).

Trema orientalis (L.) Blume Habit: Tree. Habitat: Wooded grassland, forest edges, 1-1800 m. Vouchers: FOKP 11562 (EA), Mbuni 299 (EA).

\section{F51. Moraceae}

2 Genera, 8 Species

Ficus glumosa Delile Habit: Tree. Habitat: Rocky hillsides, 450-2050 m. Vouchers: SAJIT 006836 (EA, HIB), FOKP 10936 (EA, HIB).

Ficus sur Forssk. Habit: Tree. Habitat: Riverine, Bushland, 1-2100 m. Vouchers: SAJIT 006849 (EA, HIB), FOKP 1273 \& 10949 (EA, HIB).

Ficus exasperata Vahl Habit: Tree. Habitat: Wet forest up to $1850 \mathrm{~m}$. Voucher: FOKP 920 (EA, HIB).

Ficus natalensis Hochst. Habit: Tree. Habitat: Riverine, 900-1800 m. Voucher: FOKP 1032 (EA, HIB).

Ficus thonningii Blume Habit: Tree. Habitat: Wooded grassland, 1050-2400 m. Vouchers: SAJIT 005149 (EA, HIB).

Dorstenia afromontana R.E. Fr. Habit: Herb. Habitat: Wet montane forest, 2000 2600 m. Voucher: FOKP 1814 (EA, HIB).

Dorstenia hildebrandtii var. schlechteri (Engl.) Hijman Habit: Herb. Habitat: Riverine forest, 275-2100 m. Vouchers: FOKP 11671 (EA, HIB), Mbuni 671 (EA), Napier 1915 (EA).

Dorstenia barnimiana var. tropaeolifolia (Schweinf.) Rendle Habit: Herb. Habitat: Open savannah, common everywhere in short grass, Stony upland, 1000-2100 m. Voucher: Symes 627 (EA).

\section{F52. Urticaceae}

8 Genera, 13 Species

Droguetia debilis Rendle Habit: Herb. Habitat: Highland forest, 275-2100 m. Voucher: FOKP 1875 (EA, HIB).

Droguetia iners (Forssk.) Schweinf. Habit. Herb. Habitat: Lower edge bamboo, 16003250m. Vouchers: SAJIT 004828 \& 006920 (EA, HIB), FOKP 11020 (EA, HIB). 
Elatostema monticola Hook. f. Habit: Herb. Habitat: Wet places, 1600-2800 m. Vouchers: SAJIT 006890 (EA, HIB), FOKP1735 \& 10990 (EA, HIB).

Laportea alatipes Hook. f. Habit: Herb. Habitat: Disturbed ground in wet montane forest, 1560-3000 m. Habitat: Wet mountain forest, 1560-3010 m. Voucher: FOKP 1080 (EA, HIB).

Laportea ovalifolia (Schumach. \& Thonn.) Chew. Habit: Herb. Habitat: Disturbed woodland, 900-2910 m. Voucher: SAJIT 007125 (EA, HIB).

Parietaria debilis G. Forst. Habit: Herb. Habitat: Montane forest, 1560-3010 m. Voucher: Tweedie 2698 (EA).

Pilea angolensis (Hiern) Rendle Habit: Herb. Habitat: streamsides, 1400-2800 m Voucher: SAJIT 005090 (EA, HIB).

Pilea johnstonii Oliv. Habit: Herb. Habitat: streamsides 1680-2910 m. Voucher: Part II Botany 52 (EA).

Pilea rivularis Wedd. Habit: Herb. Habitat: Montane forest, Streams \& Paths, 1480 3100 m. Voucher: SAJIT 004827 (EA, HIB).

Pilea tetraphylla (Steud.) Blume Habit: Herb. Habitat: Montane forest, 17502480 m. Voucher: Gilbert \& Meifin 6467 (EA).

Urera hypselodendron (Hochst. ex A. Rich.) Wedd. Habit: Climber. Habitat: Montane forest, moist upland forest, 1480-3100 m. Vouchers: SAJIT 004822, 005082 \& 006866 (EA, HIB), FOKP 952, 964, 11358, 11377 \& 11617 (EA, HIB), Mbuni $114 \& 617$ (EA).

Urtica massaica Mildbr. Habit: Herb. Habitat: Montane forest, 2000-3400 m. Vouchers: FOKP 961, 11312 \& 11326 (EA, HIB), Mbuni 062 (EA).

Pouzolzia parasitica (Forssk.) Schweinf. Habit: Herb. Habitat: Upland forest 1050 2100 m. Voucher: FOKP 1880 (EA, HIB).

\section{F53. Cucurbitaceae}

6 Genera, 8 Species

Coccinia adoensis (Hochst. ex A. Rich.) Cogn. Habit: Climber. Habitat: Highland grassland, 1600-2300 m. Vouchers: Napier 1899 (EA), Symes 283 (EA).

Coccinia grandis $(\mathbf{L}$.) Voigt Habit: Climber. Habitat: Riverine, 250-1650 m Vouchers: SAJIT 006918 (EA, HIB), FOKP 11018 (EA, HIB).

Cucumis ficifolius A. Rich. Habit: Climber. Habitat: Highland grassland, 10001650 m. Voucher: Symes 273 (EA).

Momordica foetida Schumach. Habit: Climber. Habitat: Grassland, Woodland 150-1650 m. Vouchers: SAJIT $004743 \& 006882$ (EA, HIB), FOKP 11293 (EA, HIB), Mbuni $656 \& 716$ (EA).

Peponium vogelii (Hook. f.) Engl. Habit: Climber. Habitat: Forest edge, 10-2600 m. Vouchers: SAJIT 006870 (EA, HIB), FOKP 1256, 1269 \& 11428 (EA, HIB), Mbuni 164 (EA), Brodhust hill 537 (EA), Napier 1975 (EA).

Trochomeria macrocarpa subsp. vitifolia R. Fern. \& A. Fern. Habit: Climber. Habitat: Grassland, 1000-2500 m. Voucher: Lindsay 130 (EA). 
Zehneria anomala C. Jeffrey Habit: Climber. Habitat: 1000-2500 m. Vouchers: FOKP 11328 (EA, HIB), Mbuni 064 (EA).

Zehneria scabra Sond. Habit: Climber. Habitat: Bushland, Forest edges, 1000-3300 m. Vouchers: SAJIT 004721, $006799 \& 006872$ (EA, HIB), FORK 933, 1005, $10899 \& 11462$ (EA, HIB), Mbuni 064 \& 198 (EA).

\section{F54. Bignoniaceae}

1 Genus, 1 Species

Stereospermum kunthianum Cham. Habit: Tree. Habitat: Rocky bushland, wooded grassland, 900-2100 m. Habitat: Voucher: Bogdan 265 (EA).

\section{F55. Celastraceae}

6 Genera, 11 Species

Catha edulis (Vahl) Endl. Habit: Tree. Habitat: Wooded grassland, 1200-2400 m. Vouchers: Munro 989 (EA), Agnew 5087 (EA).

Cassine aethiopica Thunb. Habit: Shrub. Habitat: Bushland, 900-2400 m. Vouchers: FOKP 11608 (EA, HIB), Mbuni 608 (EA).

Gymnosporia buchananii Loes. Habit: Shrub. Habitat: Grassland, 1-2650 m. Vouchers: FOKP 11294 (EA, HIB), Birch 61 \& 180 (EA).

Gymnosporia heterophylla (Eckl. \& Zeyh.) Loes. Habit: Shrub. Habitat: Dry Upland \& lowland Forest, Riverine, wooded grassland, 1150-2700 m. Vouchers: FOKP 11601 (EA, HIB), Mbuni $144 \& 601$ (EA, HIB).

Gymnosporia keniensis (Loes.) Jordaan Habit: Shrub Habitat: Dry Upland \& lowland Forest, Riverine, thickets, Woodland, 1350-2250 m. Vouchers: SAJIT 004826 (EA, HIB), Fries \& Hansen 2549 (EA).

Gymnosporia senegalensis (Lam.) Loes. Habit: Tree. Habitat: Bushed grassland, Riverine, 1-2100m. Vouchers: FOKP 11376 (EA), Buich $61 \& 180$ (EA), Lind et al. 5082 (EA), Webster 8812 (EA).

Hippocratea goetzei Loes. Habit: Shrub. Habitat: Woodland, forest thicket, 1100$2700 \mathrm{~m}$. Voucher: FOKP 1806 (EA, HIB).

Maytenus arbutifolia (Hochst. ex A. Rich.) R. Wilczek Habit: Tree. Habitat: Bushland, Grassland, 1600-2350 m. Vouchers: SAJIT 004757 (EA, HIB), FOKP 1271 (EA, HIB).

Maytenus obscura (A. Rich.) Cufod. Habit: Shrub. Habitat: Bushland, 19002550 m. Vouchers: FOKP 1089, 1235 (EA, HIB).

Maytenus undata (Thunb.) Blakelock Habit: Tree. Habitat: Evergreen forest, dry Bushland, Grassland, 1500-3200 m. Vouchers: SAJIT 004755 (EA, HIB), FOKP 1015 (EA, HIB).

Simirestis brianii N. Hallé Habit: Tree. Habitat: Forest margin, 500-2550 m. Voucher: SAJIT Z0110 (HIB). 


\section{F56. Oxalidaceae}

1 Genus, 4 Species

Oxalis anthelmintica A. Rich. Habit: Herb. Habitat: Grassland, Riverine 8402400 m. Voucher: Symes 58 (EA).

Oxalis corniculata L. Habit: Herb. Habitat: Grassland, Disturbed ground, 0-3500 m. Voucher: Townsend 2374 (EA).

Oxalis obliquifolia Steud. ex A. Rich. Habit: Herb. Habitat: Grazer pasture, Grassland, roadsides, 830-3300 m. Voucher: Thulin 81 (EA).

Oxalis radicosa A. Rich. Habit: Herb. Habitat: Weed of cultivation and disturbed ground, 830- $2500 \mathrm{~m}$. Voucher: Symes 46 (EA).

\section{F57. Rhizophoraceae}

1 Genus, 3 Species

Cassipourea malosana (Baker) Alston Habit: Tree. Habitat: Bushland, Understorey in moist forest, 750-2900 m. Voucher: SAJIT 004730 (EA, HIB).

Cassipourea rotundifolia (Engl.) Alston. Habit: Tree. Habitat: Woodland, 9001700 m. Voucher: SAJIT 007077 (EA, HIB).

Cassipourea ruwensorensis (Engl.) Alston Habit: Tree. Habitat: Evergreen forest, woodland, 1450-1600 m, Voucher: FOKP 1125 (EA, HIB).

\section{F58. Peraceae}

1 Genus, 1 Species

Clutia abyssinica Jaub. \& Spach Habit: Shrub; Habitat: Secondary bushland, 16003100 m. Vouchers: SAJIT 007042 (EA, HIB), FOKP 1035 \& 11283 (EA, HIB), Mbuni 019 (EA).

\section{F59. Euphorbiaceae}

13 Genera, 30 Species

Acalypha fruticosa Forsk. Habit: Shrub. Habitat: Lowland forest edge, 200-2000 m. Vouchers: FOKP 11676 (EA, HIB), Mbuni 676 (EA).

Acalypha volkensii Pax Habit: Shrub. Habitat: Upland grassland, 800-2700 m Vouchers: SAJIT 004732 \& 006867 (EA, HIB), FOKP 11407 (EA, HIB), Mbuni $143 \& 712 \&$ (EA).

Acalypha polymorpha Müll. Arg. Habit: Herb. Habitat: Wooded, grassland, 17002300 m. Voucher: Symes 34 (EA).

Croton dichogamus Pax Habit: Tree. Habitat: Dry bushland, Disturbed sites, 5002100 m. Vouchers: FOKP 11466 \& 11658 (EA, HIB), Mbuni $202 \& 658$ (EA). 
Croton macrostachyus Hochst. ex Delile Habit: Tree. Habitat: Wooded Grassland, dry or moist upland forest, 1350-2250 m. Vouchers: FOKP 1150 \& 11697 (EA, HIB), Mbuni $236 \& 697$ (EA), Birch $61 \& 169$ (EA).

Cordia monoica Roxb. Habit: Shrub. Habitat: Deciduous bushland, grassland, 1-2100 m. Vouchers: SAJIT Z006 (HIB).

Erythrococca bongensis Pax Habit: Tree. Habitat: Wooded Bushland, River line or thicket, 900-2100 m. Vouchers: FOKP 11430 (EA, HIB), Mbuni 166 (EA).

Erythrococca fischeri Pax Habit: Shrub. Habitat: Woodland, grassland, 1500-2300 m. Vouchers: FOKP 1028 \& 1234 (EA, HIB).

Euphorbia abyssinica J.F. Gmel. Habit: Tree. Habitat: Woodland, 2100-2400 m. Vouchers: SAJIT 006883 (EA, HIB), Haper \& Field 4975 (EA), Mabberley 497 (EA).

Euphorbia ampliphylla Pax Habit: Tree. Habitat: Woodland, grassland, 15002300 m. Voucher: Mabberley 497 (EA).

Euphorbia bongensis Kotschy \& Peyr. ex Boiss. Habit: Shrub. Habitat: Rare in grassland, 1200-2000 m. Voucher: Napper \& Tweedie 2115 (EA).

Euphorbia brevicornu Pax Habit: Herb. Habitat: Drier highlands, 2200-3600 m. Voucher: Mabberley \& McCall 254 (EA).

Euphorbia cyparissioides Pax Habit: Herb. Habitat: Grassland, 1900-2250 m. Voucher: Webster 8773 (EA).

Euphorbia depauperata Hochst. ex A. Rich. Habit: Shrub. Habitat: Highland grassland, 1900-3200 m. Voucher: Thulin \& Tidigs 224 (EA).

Euphorbia engleri Pax Habit: Herb. Habitat: Shade of montane forest, 1500-2800 m. Voucher: FOKP 1807 (EA, HIB).

Euphorbia heterospina S. Carter Habit: Shrub. Habitat: Woodland, 900-1800 m. Voucher: FOKP 1247 (EA, HIB).

Euphorbia repetita Hochst. ex A. Rich. Habit: Herb. Habitat: Grassland, 23002700 m. Vouchers: Thulin \& Tidigs 142 (EA), Agnew et al. 10530 (EA).

Euphorbia scarlatina S. Carter Habit: Shrub. Habitat: Bushland on rocky slopes, 600-2000 m. Voucher: SAJIT 006875 (EA, HIB).

Euphorbia schimperiana Scheele Habit: Herb. Habitat: Grassland, montane 16003500 m. Vouchers: SAJIT 005113 (EA, HIB), Thulin \& Tidigs 142 (EA).

Euphorbia tirucalli L. Habit: Shrub. Habitat: Dry bushland, 0-1600 m. Voucher: Tweedie 2101 (EA).

Euphorbia umbellata (Pax) Bruyns Habit: Tree. Habitat: Dry forest, riverine, 11502100 m. Voucher: Glover et al. 1969 (EA).

Macaranga capensis (Baill.) Sim Habit: Tree Habitat: Moist forest, 300-2100 m. Voucher: SAJIT 004824 (EA, HIB).

Macaranga kilimandscharica Pax Habit: Tree. Habitat: Moist upland forest, 1650 2400 m. Vouchers: SAJIT 006797 (EA, HIB), FORK 991, 1000, 10896 \& 11349 (EA, HIB), Birch 175 (EA), Buch 61 \& 175 (EA).

Margaritaria discoidea var. fagifolia (Pax) Radcl.-Sm. Habit: Herb. Habitat: Upland forest, 10-1700 m. Voucher: Obunyali \& Omondi 0226 (EA). 
Micrococca mercurialis (L.) Benth. Habit: Herb. Habitat: Dry bushland, 10-1610 m. Voucher: Bogdan 3847 (EA).

Mondia whitei (Hook. f.) Skeels. Habit: Shrub. Habitat: Forest thickets, 8002200 m. Voucher: FOKP 1718 (EA, HIB).

Neoboutonia macrocalyx Pax Habit: Tree. Habitat: Upland forest, evergreen forest, Riverbanks 1600-2700 m. Vouchers: SAJIT 005087 \& 006798 (EA, HIB), FOKP 11351 \& 11523 (EA, HIB), Mbuni 259 (EA), Tweedie 4118 (EA).

Ricinus communis L. Habit: Herb. Habitat: Bushland, Grassland, 600-3100 m. Voucher: Mas 236 (EA).

Shirakiopsis elliptica (Hochst.) Esser Habit: Tree. Habitat: Woodland, 14001800 m. Voucher: Birch 177 (EA).

Tragia brevipes Pax Habit: Herb. Habitat: Dry forest, 600-2600 m. Vouchers: SAJIT 004744 (EA, HIB), FOKP 1059 \& 11753 (EA, HIB), Mbuni 753 (EA).

\section{F60. Ochnaceae}

1 Genus, 2 Species

Ochna inermis (Forssk.) Schweinf. ex Penz. Habit: Shrub. Habitat: Bushland, 1-1450 m. Voucher: Carter \& Stannard 70 (EA).

Ochna thomasiana Engl. \& Gilg Habit: Tree. Habitat: Bushland, woodland, 15002400 m. Vouchers: FOKP 11628 (EA), Mbuni 628 (EA).

\section{F61. Phyllanthaceae}

2 Genera, 7 Species

Bridelia scleroneura Müll. Arg. Habit: Shrub. Habitat: Wooded grassland, 9001650 m. Voucher: Pole \& Erens 1519 (EA).

Phyllanthus fischeri Pax Habit: Shrub. Habitat: Woodland, Forest edge, river line bushes, 1450-2700 m. Vouchers: SAJIT 004778 (EA, HIB), FOKP 11370 (EA, HIB), Mbuni 106 (EA), Thulin \& Tidigs 122 (EA), Agnew et al. 10493 (EA), Hepper 4980 (EA).

Phyllanthus ovalifolius Forssk. Habit: Shrub. Habitat: Bushland, 1350-2450 m. Vouchers: FOKP 11459 \& 11679 (EA, HIB), Mbuni 195 \& 679 (EA).

Phyllanthus sepialis Müll. Arg. Habit: Shrub. Habitat: Riverine Woodland, Bushland, 600-1950 m. Voucher: FOKP 11384 (EA, HIB).

Phyllanthus suffrutescens Pax Habit: Herb. Habitat: Grassland 1200-2350 m. Voucher: Napier 1911 (EA).

Phyllanthus niruroides Müll. Arg. Habit: Herb. Habitat: Grassland, 1200-2350 m. Voucher: Agnew et al. 10318 (EA).

Phyllanthus boehmii Pax Habit: Herb. Habitat: Grassland moorland, 2000-3270 m. Voucher: FOKP 960 (EA, HIB). 


\section{F62. Passifloraceae}

1 Genus, 1 Species

Adenia cissampeloides (Planch. ex Hook.) Harms Habit: Climber. Habitat: Moist forest, 0-2500 m. Voucher: SAJIT 004724 (EA, HIB).

\section{F63. Salicaceae}

4 Genera, 5 Species

Casearia battiscombei R.E. Fr. Habit: Tree. Habitat: Upland moist forest, 13502400 m. Vouchers: SAJIT 006907 (EA, HIB), FOKP 1122 \& 11007 (EA, HIB), Mbuni 588 \& 618 (EA).

Dovyalis abyssinica (A. Rich.) Warb. Habit: Tree. Habitat: Upland moist forest, 1450-2600 m. Vouchers: FOKP 11365 (EA), Mbuni 101 (EA).

Dovyalis macrocalyx (Oliv.) Warb. Habit: Shrub. Habitat: Moist forest, grassland 120-2400 m. Vouchers: FOKP 11678 (EA), Mbuni 678 (EA).

Flacourtia indica (Burm. f.) Merr. Habit: Tree. Habitat: Wooded grassland, 12002400 m. Vouchers: FOKP 1264, 11288 \& 11321 (EA, HIB), Mbuni 741 (EA), Brunt 1410 (EA), Kazuaki 15 (EA), Buch 164 (EA).

Trimeria grandifolia (Hochst.) Habit: Tree. Habitat: Bushland 150-2500 m. Vouchers: FOKP 11672 (EA), Mbuni 672 (EA), Beentje 3044 (EA).

\section{F64. Violaceae}

1 Genus, 3 Species

Viola eminii (Engl.) R.E. Fr. Habit: Herb. Habitat: Forest paths \& streamside, 21003540 m. Voucher: Mabberley \& McCall 240 (EA).

Viola abyssinica Steud. ex Oliv. Habit: Herb. Habitat: Alpine, along paths, 1600$3500 \mathrm{~m}$. Voucher: Townsend 2383 (EA).

Viola villosa Walter Habit: Climber. Habitat: Forest paths \& streamside, 21003540 m. Voucher: Irwin 205 (EA).

\section{F65. Linaceae}

1 Genus, 1 Species

Linum volkensii Engl. Habit: Herb. Habitat: Upland grassland, Roadsides \& waste places, 1100-2900 m. Voucher: Agnew et al. 10504 (EA).

\section{F66. Hypericaceae}

1 Genus, 6 Species

Hypericum kiboense Oliv. Habit: Shrub. Habitat: Bamboo zone, 2250-3250 m. Voucher: Mabberley 582 (EA). 
Hypericum lalandii Choisy. Habit: Herb. Habitat: Grassland, 1500-2300 m. Voucher: Symes 615 (EA).

Hypericum peplidifolium A. Rich. Habit: Herb. Habitat: Subalpine grassland, 13503500 m. Voucher: Napier 1927 (EA), Mabberley 7 (EA), McCall 192 (EA), Symes 45 (EA).

Hypericum quartinianum A. Rich. Habit: Shrub. Habitat: Rocky stream-banks, 1500-2250 m. Voucher: FOKP 11268 (EA, HIB).

Hypericum revolutum Vahl Habit: Shrub. Habitat: Heath zone, 2700-3500 m. Vouchers: SAJIT 004812 (EA, HIB), FOKP 992, 1087 \& 11773 (EA, HIB), Mbuni 001 (EA), Knox 4174 (EA), Kokwaro 2532 (EA).

Hypericum scioanum Chiov. Habit: Herb. Habitat: Montane forest, 1850-3500 m. Vouchers: SAJIT 005120 (EA, HIB), Knox $3451 \& 3364$ (EA).

\section{F67. Geraniaceae}

2 Genera, 4 Species

Geranium vagans Baker Habit: Herb. Habitat: Grassland in montane, 2640-3500 m. Voucher: Beentje 3072 (EA).

Geranium aculeolatum Oliv. Habit: Herb. Habitat: Montane forest 2640-3500 m. Vouchers: SAJIT 005030 (EA, HIB), Mabberley 255 (EA).

Geranium arabicum Forssk. Habit: Herb. Habitat: Montane forest, 1800-3550 m. Voucher: FOKP 1300, Napier 1972 (EA).

Pelargonium alchemilloides (L.) Aiton Habit: Herb. Habitat: Wooded grassland montane forest edge, 1000-2100 m. Voucher: FOKP 1825 (EA).

\section{F68. Melianthaceae}

1 Genus, 1 Species

Bersama abyssinica Fresen. Habit: Tree. Habitat: Dry \& wet montane forest, 1150 2550 m. Vouchers: SAJIT 004818, $005071 \& 007049$ (EA, HIB), FOKP 941, $1263,11284 \& 11410$ (EA, HIB), Mbuni $146 \& 708$ (EA).

\section{F69. Combretaceae}

2 Genera, 6 Species

Combretum apiculatum Sond. Habit: Tree. Habitat: Wooded grassland, 2501750 m. Voucher: SAJIT 004849 (EA).

Combretum collinum subsp. binderianum (Kotschy) Okafa Habit: Tree. Habitat: Wooded grassland, 700-2200 m. Voucher: Lucas 156 (EA).

Combretum capituliflorum Fenzl ex Schweinf. Habit: Liana. Habitat: Wooded grassland, Riverine forest, 900-1100 m. Voucher: Wilson 310 (EA).

Combretum molle R. Br. ex G. Don Habit: Tree. Habitat: Wooded grassland, 1502100 m. Vouchers: FOKP 1102, $11270 \& 11682$ (EA, HIB). 
Terminalia boivinii Tul. Habit: Tree. Habitat: Woodland, 700-1900 m. Voucher: SAJIT Z0055 (EA, HIB).

Terminalia brownii Fresen. Habit: Tree. Habitat: Bushland, Woodland 700-1900 m. Voucher: FOKP 11342 (EA, HIB).

\section{F70. Lythraceae}

2 Genera, 2 Species

Rotala tenella (Guill. \& Perr.) Hiern Habit: Herb. Habitat: Grassland, 12502100 m. Vouchers: SAJIT 006913 (EA, HIB), FOKP 11013 (EA, HIB).

Woodfordia uniflora (A. Rich.) Koehne Habit: Shrub. Habitat: Bushland, Rocky slopes, along dry rivers, 1350-2300 m. Voucher: Gardner 3717 (EA).

\section{F71. Onagraceae}

2 Genera, 4 Species

Epilobium hirsutum L. Habit: Herb. Habitat: Swamps, upland grassland, 1800 2450 m. Vouchers: FOKP 11332 \& 11720 (EA, HIB), Mbuni 068, 720 (EA).

Epilobium salignum Hausskn. Habit: Herb. Habitat: Swampy places, 1800-3550 m. Voucher: Brodhurst 505 (EA).

Epilobium stereophyllum Fresen. Habit: Herb. Habitat: Upland grassland, 26003500 m. Vouchers: Symes 626 (EA), Thulin et al. 106 (EA), Muthama et al. 170 (EA). Ludwigia abyssinica A. Rich. Habit: Herb. Habitat: Swampy places, 1000-2300 m. Vouchers: FOKP 974 (EA, HIB), Symes 517 (EA).

\section{F72. Myrtaceae}

2 Genera, 3 Species

Psidium guajava L. Habit: Tree. Habitat: Bushland, 1000-2500 m. Vouchers: Mbuni 207 (EA), FOKP 11471 (EA).

Syzygium guineense (Willd.) DC. Habit: Tree. Habitat: Wooded grassland, Riverine, 1-2500 m. Vouchers: SAJIT 005086 (EA, HIB), FOKP 11612 (EA), Mbuni 612 (EA), Thomas 2110 (EA), Dale 902 (EA).

Syzygium cordatum Hochst. ex Krauss Habit: Tree. Habitat: Riverine, 1-2500 m. Vouchers: FOKP 11272, 11443 \& 11669 (EA, HIB), Mbuni 179 \& 669(EA).

\section{F73. Melastomataceae}

2 Genera, 6 Species

Antherotoma naudinii Hook. f. Habit: Herb. Habitat: Shallow soils, watercourses, 500-2300 m. Voucher: Bogdan 5280 (EA).

Dissotis debilis Triana Habit: Herb. Habitat: Swampy grassland, 10-1800 m. Voucher: FOKP 1242 (EA, HIB). 
Dissotis speciosa Taub. Habit: Shrub. Habitat: Swampy areas, 1000-2250 m. Vouchers: Verdcourt 734 (EA), Webster 8749, Gardner 3718 (EA).

Dissotis canescens (E. Mey. ex Graham) Hook. f. Habit: Herb. Habitat: Swampy grassland, 800-2200 m. Vouchers: Webster 748 \& 8747 (EA).

Dissotis senegambiensis (Guill. \& Perr.) Triana Habit: Herb. Habitat: Grassland, 1350-2650 m. Voucher: Webster 8746 (EA).

Dissotis irvingiana Hook. Habit: Herb. Habitat: Swampy grassland, 800-2200 m. Voucher: Webster 8745 (EA).

\section{F74. Penaeaceae}

1 Genera, 1 Species

Olinia rochetiana A. Juss. Habit: Tree. Habitat: Dry upland forest, 1700-3050 m. Vouchers: FOKP 1024 \& 1216 (EA, HIB), Mbuni 007 \& 745 (EA), Rauh 577 (EA), Part II Botany 47 (EA).

\section{F75. Burseraceae}

1 Genus, 3 Species

Commiphora eminii Engl. Habit: Tree. Habitat: Dry evergreen forest, 600-1000 m. Voucher: SAJIT Z0062 (EA, HIB).

Commiphora habessinica (O. Berg) Engl. Habit: Shrub. Habitat: Bushed grassland, 500-1900 m. Voucher: Tanaka 234 (EA).

Commiphora holtziana Engl. Habit: Tree. Habitat: Bushland, 20-1100 m. Voucher: FOKP 11341 (EA, HIB).

\section{F76. Anacardiaceae}

4 Genera, 10 Species

Lannea fulva (Engl.) Engl. Habit: Shrub. Habitat: Bushland, Wooded grassland, 950-1900 m. Voucher: FOKP 11335 (EA, HIB).

Lannea schimperi (Hochst. ex A. Rich.) Engl. Habit: Tree. Habitat: Wooded grassland, 1300-1900 m. Voucher: FOKP1818 (EA, HIB).

Lannea triphylla (Hochst. ex A. Rich.) Engl. Habit: Shrub. Habitat: Wooded grassland, 50-1650 m. Vouchers: FOKP 11674 (EA), Mbuni 674 (EA).

Ozoroa insignis Delile Habit: Tree. Habitat: Wooded grassland, 1-2500 m. Vouchers: SAJIT 004740 (EA, HIB), FOKP 11746 (EA, HIB), Mbuni 746 (EA), Kefri 24 (EA).

Ozoroa insignis subsp. reticulata (Baker f.) J.B. Gillett Habit: Tree. Habitat: Wooded grassland, 1500-2500 m. Voucher: Rindsay 169 (EA).

Rhus longipes Engl. Habit: Tree. Habitat: Wooded grassland, 1000-2400 m. Vouchers: SAJIT 006808 (EA, HIB), FOKP 10908 \& 11308 (EA, HIB). 
Searsia natalensis (Bernh. ex C. Krauss) F.A. Barkley Habit: Shrub. Habitat: Dry forest wooded grassland, forest margin, forest thicket, 1050-2700 m. Vouchers: FOKP 1096, 11436 \& 11290 (EA, HIB), Mbuni 172 (EA).

Searsia pyroides (Burch.) Moffett Habit: Tree. Habitat: Wooded grassland; 12002700 m. Vouchers: FOKP 11352 \& 11650 (EA, HIB), Mbuni 088 \& 650 (EA).

Searsia tenuinervis (Engl.) Moffett Habit: Shrub. Habitat: Wooded grassland, 850 1500 m. Vouchers: FOKP 11740 (EA, HIB), Mbuni 740 (EA).

Searsia ruspolii (Engl.) Moffett Habit: Shrub. Habitat: Evergreen bushland, 1500 2400 m. Vouchers: SAJIT 006871 (EA, HIB), FOKP 1021, 11324 \& 11575 (EA, HIB), Mbuni 312 (EA), Kokwaro 2537 (EA).

\section{F77. Sapindaceae}

8 Genera, 9 Species

Allophylus abyssinicus (Hochst.) Radlk. Habit: Tree. Habitat: Moist forest, river line, 1000-2550 m. Vouchers: SAJIT 006927 (EA, HIB), FOKP 1130, 11359 \& 11542 (EA, HIB), Mbuni 279 (EA).

Cardiospermum grandiflorum Sw. Habit: Climber. Habitat: Upland forest edges, 750-1800 m. Vouchers: Bogdan 3834 (EA), Mabberley \& McCall 99 (EA).

Deinbollia borbonica Scheff. Habit: Tree. Habitat: Secondary bushland, 12002550 m. Vouchers: FOKP 1068 (EA, HIB).

Dodonaea viscosa (L.) Jacq. Habit: Shrub. Habitat: Secondary bushland, 10002700 m. Vouchers: SAJIT 004844 (EA, HIB), FOKP 11295 (EA, HIB).

Dodonaea viscosa subsp. angustifolia (L. f.) J.G. West Habit: Shrub. Habitat: Secondary bushland, 1000-2700 m. Vouchers: FOKP 1278 \& 11686 (EA, HIB), Mbuni 686 (EA).

Haplocoelum foliolosum (Hiern) Bullock Habit: Tree. Habitat: Secondary bushland, 900-1800 m. Voucher: SAJIT Z0048 (HIB).

Lecaniodiscus fraxinifolius subsp. vaughanii (Dunkley) Friis Habit: Tree. Habitat: Woodland, 1-1200 m. Voucher: Dale 1093 (EA).

Lepisanthes senegalensis (Poir.) Leenh. Habit: Tree. Habitat: Woodland, riverine forest, 1-1900 m. Voucher: Tweedie 2961 (EA).

Pappea capensis Eckl. \& Zeyh. Habit: Tree. Habitat: Bushland, Wooded grassland, 1050-2300 m. Voucher: Aggundey 5 (EA).

\section{F78. Rutaceae}

5 Genera, 6 Species

Clausena anisata (Willd.) Hook. f. ex Benth. Habit: Shrub. Habitat: Secondary bushland, 1-2650 m. Vouchers: FOKP 11460, 11554 \& 11657 (EA, HIB), Mbuni 196, $291 \& 657$ (EA).

Harrisonia abyssinica Oliv. Habit: Tree. Habitat: Dry bushland, wooded grassland, 1-1650 m. Voucher: Tweedie 3793 (EA). 
Teclea nobilis Delile Habit: Tree. Habitat: Wooded grassland, 1050-2550 m. Vouchers: FOKP 11637 (EA, HIB), Mbuni 637 (EA), Lind 5083 (EA).

Toddalia asiatica (L.) Lam. Habit: Liana. Habitat: Forest margin, grassland thicket, 1200-3000 m. Vouchers: SAJIT 005074 \& Z0004 (EA, HIB), FOKP 11401 \& 957 (EA, HIB), Nappier 1994 (EA).

Vepris nobilis (Delile) Mziray Habit: Tree. Habitat: Woodland, 1-1400 m. Vouchers: SAJIT 004823 \& 005083 (EA, HIB), FOKP 1028 (EA, HIB), Mbuni 194 (EA).

Vepris simplicifolia (Engl.) Mziray Habit: Tree. Habitat: Woodland, 1-1400 m. Voucher: FOKP 1872 (EA, HIB).

\section{F79. Meliaceae}

2 Genera, 2 Species

Ekebergia capensis Sparrm. Habit: Tree. Habitat: Dry forest and riverine, 13002600 m. Voucher: SAJIT 005079 (EA, HIB).

Lepidotrichillia volkensii (Gurke) Leroy Habit: Tree. Habitat: Dry forest, Forest margin, 1500-2000 m. Vouchers: FOKP 1068, $11411 \& 11623$ (EA, HIB), Mbuni $147 \& 623$ (EA).

\section{F80. Malvaceae}

10 Genera, 34 Species

Abutilon bidentatum Hochst. ex A. Rich. Habit: Herb. Habitat: Savanna, rocky places, 500-2000 m. Voucher: Lye 9057 (EA).

Abutilon holstii K. Schum. ex Engl. Habit: Shrub. Habitat: Bushland up to 2000 m. Voucher: Webster 8758 (EA).

Abutilon hirtum (Lam.) Sweet Habit: Herb. Habitat: Dry Bushland, 1-1350 m. Voucher: Mas 201 (EA).

Abutilon longicuspe Hochst. ex A. Rich. Habit: Shrub. Habitat: Upland forest, 1650-3300 m. Voucher: SAJIT 004752 (EA, HIB).

Abutilon mauritianum (Jacq.) Medik. Habit: Shrub. Habitat: Riverine, 0-2300 m. Voucher: Lindsay 2 (EA).

Dombeya burgessiae Gerrard ex Harv. Habit: Tree. Habitat: Riverine, bushland, Woodland, thickets, 1200-3000 m. Vouchers: SAJIT 004764 (EA, HIB), FOKP 1286 \& 11572 (EA, HIB), Mbuni 309 (EA).

Dombeya torrida (J.F. Gmel.) Bamps Habit: Tree. Habitat: Hagenia forest, 1850 2700 m. Vouchers: FOKP 937 (EA, HIB), Lind et al. 5080 (EA).

Dombeya quinqueseta (Delile) Exell Habit: Tree. Habitat: Wooded grassland, 16501750 m. Voucher: Webster 8757 (EA).

Dombeya torrida subsp. erythroleuca (K. Schum.) Seyani Habit: Tree. Habitat: Secondary bushland, 1850-2700 m. Voucher: FOKP 11363 (EA, HIB).

Dombeya rotundifolia (Hochst.) Planch. Habit. Tree. Habitat: Wooded grassland, 900-2250 m. Voucher: Webster 8757 (EA). 
Hibiscus aponeurus Sprague \& Hutch. Habit. Shrub. Habitat: Grassland, 6002600 m. Vouchers: Agnew et al. 10300 (EA), Lindsay 164 (EA).

Hibiscus berberidifolius A. Rich. Habit. Shrub. Habitat: Upper forest edge, 1500 3600 m. Vouchers: SAJIT 007069 (EA, HIB), FOKP 973 (EA, HIB), Mbuni 080 (EA).

Hibiscus diversifolius Jacq. Habit: Shrub. Habitat: Disturbed places, 1330-2800 m. Voucher: Brodhurst-Hill 8765 (EA).

Hibiscus fuscus Garcke Habit: Shrub. Habitat: Permanent grassland, Disturbed places, 1400-2650 m. Vouchers: FOKP 11291 \& 11498 (EA, HIB), Mbuni $234 \& 687$ (EA), Maas 6358 (EA), Symes 72 (EA).

Hibiscus ludwigii Eckl. \& Zeyh. Habit. Shrub. Habitat: Cleared forest, Roadside, 1800-3000 m. Vouchers: SAJIT 004472 \& 006923 (EA, HIB), FOKP 11504 (EA), Mbuni 059, 117 \& 240B (EA), Mabberley \& McCall 2003 (EA).

Hibiscus micranthus L. f. Habit: Shrub. Habitat: Grassland, 1500-2900 m. Vouchers: Agnew et al. 10333 (EA), Bogdan 4769 (EA), Mora 4039 (EA), Mus 97 (EA), Webster 8763 (EA).

Grewia similis K. Schum. Habit. Shrub. Habitat: Bushland, Grassland, 700-2250 m. Vouchers: FOKP 1025 \& 11667 (EA, HIB), Mbuni 667 (EA), Webster 8752 (EA).

Grewia tephrodermis K. Schum. Habit. Tree. Habitat: Bushland, 700-2250 m. Voucher: Mbuni 226 \& 672 (EA), FOKP $11490 \& 11672$ (EA, HIB).

Grewia densa K. Schum. Habit. Shrub. Habitat: Bushland, 350-1500 m. Voucher: FOKP 11726 (EA, HIB), Mbuni 726 (EA).

Kosteletzkya adoensis (Hochst. ex A. Rich.) Mast. Habit: Shrub. Habitat: Grassland, 1200-2650 m. Voucher: FOKP 1294 (EA, HIB).

Kosteletzkya begoniifolia Ulbr. Habit: Shrub. Habitat: Grassland, 750-2100 m. Habitat: Voucher: FOKP 985 (EA, HIB).

Malva verticillata L. Habit: Herb. Habitat: Grassland, 1500-3500 m. Voucher: Thulin \& Tidings 241 (EA).

Pavonia burchellii (DC.) R.A. Dyer. Habit. Shrub. Habitat: Commiphora Woodland, 100-2300 m. Voucher: Symes 65 (EA).

Pavonia propinqua Garcke Habit. Shrub. Habitat: Commiphora Bushland, 5501400 m. Voucher: SAJIT 004720 (EA, HIB).

Pavonia urens Cav. Habit: Shrub. Habitat: Riverine Woodland, 1200-3000 m. Vouchers: FOKP 975 \& 11403 (EA, HIB), Mbuni 073 \& 103 (EA), Symes 513 (EA), Brown 12809 (EA), Kerfoot 8771 (EA).

Sida acuta Burm. f. Habit: Herb. Habitat: Grassland, 5-2400 m. Disturbed places on grassland forest, 5-1300 m. Vouchers: Mbuni 245 (EA), FOKP 11509 (EA, HIB).

Sida schimperiana Hochst. ex A. Rich. Habit: Shrub. Habitat: Dry grassland, bushland forest edges, 1390-2480 m. Voucher: Mbuni 245 (EA).

Sida ovata Forssk. Habit. Herb. Habitat: Dry grassland, 15-2500 m. Voucher: Leippert 5042 (EA).

Sida rhombifolia L. Habit. Herb. Habitat: Grassland, 900-2600 m. Vouchers: SAJIT 004735 (EA, HIB), Napier 1901 (EA), Agnew et al. 10275 (EA). 
Sida ternata L. f. Habit. Herb. Habitat: Bamboo forest, montane rain forest, 20003280 m. Vouchers: FOKP 11545 (EA, HIB), Mbuni 282 (EA), Symes 100 (EA).

Sparmannia ricinocarpa (Eckl. \& Zeyh.) Kuntze Habit: Shrub. Habitat: Forest edge, 2015-3380 m. Vouchers: FOKP 11711 (EA, HIB), Mbuni 711 (EA).

Triumfetta rhomboidea Jacq. Habit. Shrub. Habitat: Upland forest, 1100-2200 m. Vouchers: FOKP 11445 (EA, HIB), Mbuni 181 (EA), Symes 215 (EA).

Triumfetta brachyceras K. Schum. Habit. Shrub. Habitat: Forest edge, roadside, 1500-3000 m. Voucher: Hepper \& Field 4983 (EA).

Triumfetta cordifolia A. Rich. Habit. Shrub. Habitat: Montane forest edges, 10002600 m. Vouchers: FOKP 11333 (EA, HIB), Mbuni 069 (EA).

\section{F81. Thymelaeaceae}

2 Genera, 8 Species

Englerodaphne subcordata (Meisn.) Engl. Habit: Shrub. Habitat: Dry forest, Bushland, 1450-2500 m. Voucher: SAJIT 005147 (EA, HIB).

Gnidia chrysantha (Solms ex Schweinf.) Gilg Habit: Herb. Habitat: Woodland, 2000-2350 m. Voucher: Symes 382 (EA).

Gnidia fastigiata Rendle Habit: Shrub. Habitat: Subalpine grassland, 2300-3000 m. Vouchers: Dale 3175 (EA), Townsend 2370 (EA).

Gnidia glauca (Fresen.) Gilg Habit: Tree. Habitat: Forest margin, Bamboo Zone, 2250-3300 m. Vouchers: FOKP 1244 \& 11266 (EA), Mbuni 002 (EA), Birnie 480 (EA), Kokwaro 2524 (EA).

Gnidia kraussiana Meisn. Habit: Herb. Habitat: Grassland \& rocky hillside, 1650$2650 \mathrm{~m}$. Voucher: Lind 2845 (EA).

Gnidia latifolia (Oliv.) Gilg. Habit: Shrub. Habitat: Bushed grassland, 1-1950 m. Vouchers: SAJIT 006814 (EA, HIB), FOKP 10914 (EA, HIB).

Gnidia lamprantha Gilg. Habit: Shrub. Habitat: Woodland, Bushland, 12002150 m. Vouchers: FOKP 11267 (EA, HIB), Webster 8740 (EA).

Struthiola thomsonii Oliv. Habit: Shrub. Habitat: Rocky grassland, 2000-3500 m. Vouchers: SAJIT 004793 (EA, HIB), Mbuni 104 (EA), Bogdan 4516 (EA), Wamukoya 128 (EA), Rauh 676 (EA).

\section{F82. Moringaceae}

1 Genus, 1 Species

Moringa oleifera Lam. Habit: Tree. Habitat: Grassland, Bushland, 15-1350 m. Voucher: Bogdan 4517A (EA).

\section{F83. Salvadoraceae}

1 Genus, 1 Species

Salvadora persica L. Habit: Herb. Habitat: Grassland, Bushland, 1-1850 m. Voucher: FOKP 1253 (EA, HIB). 
F84. Resedaceae

1 Genus, 1 Species

Caylusea abyssinica (Fresen.) Fisch. \& C.A. Mey. Habit: Herb. Habitat: Disturbed, medium altitude, 1100-2800 m. Vouchers: FOKP 11511 (EA, HIB), Mbuni 247 (EA).

\section{F85. Capparaceae}

4 Genera, 6 Species

Cleome gynandra L. Habit: Herb. Habitat: Dry bushland, 1-2200 m. Voucher: Mabberley \& McCall 100 (EA).

Cadaba farinosa Forssk. Habit: Shrub. Habitat: Bushland, 1-1900 m. Vouchers: FOKP 1254 \& 11338 (EA, HIB).

Capparis erythrocarpos Isert. Habit: Shrub. Habitat: Bushed or wooded grassland, 1100-1800 m. Voucher: Tweedie 1931 (EA).

Maerua decumbens (Brongn.) DeWolf Habit: Shrub. Habitat: Bushland, 1-1800 m. Voucher: FOKP 1248 (EA, HIB).

Maerua parvifolia Pax Habit: Shrub. Habitat: Bushland, 700-1650 m. Voucher: Tweedie 1571 (EA).

Maerua triphylla A. Rich. Habit: Shrub. Habitat: Bushland, 1-2300 m. Vouchers: SAJIT Z0060 (HIB), FOKP 11304 \& 11332 (EA, HIB).

\section{F86. Brassicaceae}

4 Genera, 4 Species

Arabis alpina L. Habit: Herb. Habitat: Highland grassland, 2800-3500 m. Voucher: Mabberley \& McCall 211 (EA).

Cardamine africana L. Habit: Herb. Habitat: Highland grassland, 2200-3300 m. Vouchers: FOKP 1723 \& 11615 (EA, HIB), Mbuni 615 (EA).

Nasturtium officinale R. Br. Habit: Herb. Habitat: Highland streamside, 15002700 m. Voucher: FOKP 1731 (EA, HIB).

Thlaspi alliaceum L. Habit: Herb. Habitat: Bamboo forest, 3000-3500 m. Voucher: Mabberley \& McCall 258 (EA).

\section{F87. Santalaceae}

4 Genera, 5 Species

Osyridicarpos schimperianus (Hochst. ex A. Rich.) A. DC. Habit: Shrub. Habitat: Rocky site, thickets Bushland, Grassland, 1550-2415 m. Vouchers: Mbuni 750 (EA), FOKP 11750 (EA).

Osyris lanceolata Hochst. \& Steud. Habit: Shrub. Habitat: Rocky site, forest thicket, bushland, grassland, 900-2550 m. Vouchers: SAJIT 004835 (EA, HIB), FOKP 1093, 1094, $11269 \& 11270$ (EA, HIB), Mbuni 006 (EA). 
Thesium radicans Hochst. ex A. Rich. Habit: Herb. Habitat: Grassland, 18003000 m. Voucher: SAJIT 006858 (EA, HIB).

Thesium ussanguense Engl. Habit: Herb. Habitat: Grassland, around $2100 \mathrm{~m}$. Voucher: Friis \& Hansen 2508 (EA).

Viscum tuberculatum A. Rich. Habit: Parasitic herb. Habitat: Dry woodland forest, 1650-2400 m. Vouchers: SAJIT 006852 \& 007078 (EA, HIB), FOKP 1106 \& 10952 (EA, HIB), Mbuni 727 (EA).

\section{F88. Loranthaceae}

6 Genera, 11 Species

Englerina woodfordioides (Schweinf.) Balle. Habit: Shrub. Habitat: Montane forest, 1600-3050 m. Vouchers: SAJIT 006885 \& 006905 (EA, HIB), FOKP 1029 \& 11631 (EA, HIB), Mbuni 631 (EA), Bogdan 4512 (EA), Mabberley 33 (EA).

Erianthemum dregei (Eckl. \& Zeyh.) Tiegh. Habit: Shrub. Habitat: Bushland, 0-2950 m. Vouchers: SAJIT 004846 \& 004847 (EA, HIB), FOKP 1103 \& 11329 (EA, HIB).

Oncocalyx fischeri (Engl.) M.G. Gilbert Habit: Shrub. Habitat: Bushland, 5502100 m. Vouchers: Lucas 175 (EA), Kazuaki 28 (EA).

Oncocalyx sulfureus (Engl.) Wiens \& Polhill Habit: Shrub. Habitat: Dry upland forest, Bushland, 550-2680 m. Voucher: SAJIT Z0024 (EA, HIB).

Oncocalyx ugogensis (Engl.) Wiens \& Polhill Habit: Shrub. Habitat: Dry Bushland 10-1425 m. Voucher: Carter \& Stannard 79 (EA).

Phragmanthera dschallensis (Engl.) M.G. Gilbert. Habit: Shrub. Habitat: Wooded grassland, 1400-1900 m. Vouchers: SAJIT 006865 (EA, HIB), FOKP 11373 (EA, HIB), Mbuni 109 (EA), Wiens 4504 (EA).

Phragmanthera usuiensis (Oliv.) M.G. Gilbert. Habit: Shrub. Habitat: Montane forest, 1150-2600 m. Vouchers: SAJIT 004841 (EA, HIB), FOKP 11323 (EA, HIB).

Plicosepalus curviflorus (Benth. ex Oliv.) Tiegh. Habit: Shrub. Habitat: Wooded grassland, 500-2320 m. Vouchers: FOKP 11340 \& 1258 (EA, HIB), Webster 8813 (EA).

Plicosepalus meridianus (Danser) Wiens \& Polhill Habit: Shrub. Habitat: Dry bushland, 100-1550 m. Vouchers: Tweedie 2974 (EA), Archer 494 (EA).

Plicosepalus sagittifolius (Engl.) Danser Habit: Shrub. Habitat: Dry bushland \& wooded grassland, 150-2100 m. Voucher: Lucas 186 (EA) .

Tapinanthus buvumae (Rendle) Danser Habit: Climber Habitat: Wooded grassland, up to 1980 m. Vouchers: SAJIT $004831 \& 005060$ (EA, HIB).

F89. Plumbaginaceae

1 Genus, 1 Species

Plumbago zeylanica L. Habit: Shrub. Habitat: Dry bushland, 700-1860 m. Voucher: Mabberley 87 (EA). 


\section{F90. Polygonaceae}

3 Genera, 9 Species

Harpagocarpus snowdenii Hutch. \& Dandy Habit: Herb. Habitat: Highland forest up to 1650 m. Vouchers: FOKP 11541 (EA, HIB), Mbuni 278 (EA).

Persicaria decipiens (R. Br.) K.L. Wilson Habit: Herb. Habitat: Waterside grassland, 1000-3170 m. Vouchers: FOKP 978 \& 1129 (EA, HIB).

Persicaria nepalensis (Meisn.) Miyabe Habit: Shrub. Habitat: Wet forest zones, 2250 3500 m. Vouchers: SAJIT 004740 (EA HIB), Mbuni 043 (EA), Ivens 1250 (EA).

Persicaria senegalensis (Meisn.) Soják Habit: Herb. Habitat: Waterside grassland up to 2850 m. Vouchers: FOKP 11730 (EA, HIB), Mbuni 730(EA).

Persicaria setosula (A. Rich.) K.L. Wilson Habit: Herb. Habitat: Higher altitudes in waterside, 1330-3000 m. Vouchers: FOKP 1734, 11307 \& 11357 (EA, HIB), Mbuni 043 \& 093 (EA).

Persicaria strigosa (R. Br.) Nakai Habit: Herb. Habitat: Grassland, 1600-3000 m. Voucher: Ivens 1250 (EA).

Rumex abyssinicus Jacq. Habit: Herb. Habitat: Upper forest levels, 1600-2800 m. Voucher: Townsend 2385 (EA).

Rumex steudelii Hochst. ex A. Rich. Habit: Herb. Habitat: Upper forest, streamside, around 1200 m. Vouchers: FOKP 1203 \& 1225 (EA, HIB), Mbuni 089 (EA, HIB).

Rumex usambarensis (Dammer) Dammer. Habit: Herb. Habitat: Secondary shrubland, 900-2300 m. Vouchers: FOKP 11565 (EA, HIB), Mbuni 302 (EA).

\section{F91. Caryophyllaceae}

4 Genera, 7 Species

Cerastium afromontana T.C.E. Fr. Habit: Herb. Habitat: Grassland, 2300-3500 m. Voucher: Symes 648 (EA).

Cerastium lanceolatum (Poir.) Volponi Habit: Herb. Habitat: Wet Grassland, 15003500 m. Vouchers: SAJIT 006860 (EA, HIB), FOKP 1063 (EA, HIB).

Cerastium octandrum Hochst. ex A. Rich. Habit: Herb. Habitat: Grassland, 25003500 m. Voucher: Knight 95 (EA).

Lychnis crassifolia (T.C.E. Fr.) M. Popp Habit: Herb. Habitat: Grassland, 31503350 m. Voucher: Thulin \& Tidigs 232 \& 3150 (EA).

Silene gallica L. Habit: Herb. Habitat: Alpine zone, 1900-3500 m. Vouchers: FOKP $1801 \& 11306$ (EA, HIB), Mbuni 042 (EA), Symes 125 (EA).

Stellaria mannii Hook. f. Habit: Herb. Habitat: Wet highland forest, 1550-2500 m. Vouchers: FOKP $1121 \& 1224$ (EA, HIB).

Stellaria sennii Chiov. Habit: Herb. Habitat: Wet highland forest, 2020-3500 m. Vouchers: Mabberley \& McCall 203 (EA), Thulin \& Tidigs 238 (EA). 


\section{F92. Amaranthaceae}

7 Genera, 11 Species.

Achyranthes aspera L. Habit: Herb. Habitat: Woodland, disturbed dry places, 0-3080 m. Vouchers: Mbuni 81 (EA), FOKP 11345 (EA, HIB).

Achyranthes aspera var. sicula L. Habit: Herb. Habitat: Disturbed dry places, 0-3080 m. Voucher: Part II Botany 15 (EA)

Aerva lanata (L.) Juss. Habit: Shrub. Habitat: Open ground, 1-2200 m. Vouchers: FOKP 11485 (EA, HIB), Mbuni 221 (EA).

Amaranthus hybridus L. Habit: Herb. Habitat: Disturbed ground, 1500-2600 m. Vouchers: FOKP 11516 (EA, HIB), Mbuni 252 (EA).

Amaranthus spinosus L. Habit: Herb Habitat: Wooded grassland, 0-2000 m. Vouchers: FOKP 11486 (EA, HIB), Mbuni 222 (EA).

Celosia anthelminthica Asch. Habit: Climber. Habitat: Dry bushland 500-2300 m. Voucher: Mabberley \& McCall 107 (EA).

Chenopodium opulifolium Schrad. ex W.D.J. Koch \& Ziz Habit: Herb. Habitat: Cultivated land, roadside, waste places, 1600-2300 m. Vouchers: FOKP 11342 (EA, HIB), Mbuni 078 (EA).

Cyathula cylindrica Moq. Habit: Herb. Habitat: Rocky scarps with bushland, 13003240 m. Voucher: SAJIT 007115 (EA, HIB).

Cyathula polycephala Baker Habit: Herb. Habitat: Grassland, woodland, 16003000 m. Voucher: FOKP 1126 (EA, HIB).

Cyathula uncinulata (Schrad.) Schinz. Habit: Herb. Habitat: Forest edges, 15002900 m. Vouchers: FOKP 1126 (EA, HIB), Part II Botany 26 (EA).

Pupalia lappacea (L.) Juss. Habit: Herb. Habitat: Dry bushland, 10-2060 m. Vouchers: FOKP $11693 \& 11721$ (EA, HIB), Mbuni $693 \& 721$ (EA).

\section{F93. Phytolaccaceae}

1 Genus, 2 Species

Phytolacca dodecandra L'Hér. Habit: Shrub. Habitat: Moist forest margin, Riverine forest, 1650-2450 m. Vouchers: SAJIT $004749 \& 006884$ (EA, HIB), FOKP 969 $\& 1038$ (EA, HIB).

Phytolacca octandra L. Habit: Shrub. Habitat: Moist forest margin, 1500-2400 m. Vouchers: SAJIT 004776, 005085 (EA, HIB), FOKP $1111 \& 11327$ (EA, HIB), Mbuni 063 (EA).

\section{F94. Nyctaginaceae}

1 Genus, 1 Species

Boerhavia plumbaginea Cav. Habit: Herb. Habitat: Grassland, 600-2030 m. Voucher: FOKP 1250 (EA, HIB). 


\section{F95. Molluginaceae}

1 Genus, 1 Species

Corrigiola litoralis L. Habit: Herb. Habitat: Mountain grassland roadside, 23503500 m. Voucher: Mabberley \& McCall 220 (EA).

\section{F96. Basellaceae}

1 Genus, 1 Species

Basella alba L. Habit: Shrub. Habitat: Riverine forest margin and hedges, 15003000 m. Vouchers: FOKP 982 \& 11355 (EA, HIB), Mbuni 091 (EA).

\section{F97. Talinaceae}

1 Genus, 2 Species

Talinum caffrum (Thunb.) Eckl. \& Zeyh. Habit: Herb. Habitat: Bushland, 10002000 m. Voucher: Greenway 9890 (EA).

Talinum arnotii Hook. f. Habit: Herb. Habitat: Grassland, 800-2000 m. Voucher: Carter \& Stannard 62 (EA).

\section{F98. Cactaceae}

1 Genus, 1 Species

Rhipsalis baccifera (J.S. Muell.) Stearn Habit: Epiphyte. Habitat: Dry forest, 1502300 m. Voucher: FOKP 988 (EA, HIB).

\section{F99. Cornaceae}

1 Genus, 1 Species

Cornus volkensii Harms Habit: Tree. Habitat: Bamboo zone, wet upland, 21003200 m. Vouchers: SAJIT 006804 \& 007123 (EA, HIB), FOKP 1058, 11445, $10904 \& 11544$ (EA, HIB), Mbuni $181 \& 281$ (EA).

\section{F100. Balsaminaceae}

1 Genus, 9 Species

Impatiens elegantissima Gilg Habit: Herb. Habitat: Streamsides in wet forest, 10002750 m. Vouchers: Hepper \& Field 5017, Maas 4774 (EA), Webster 8737 (EA).

Impatiens hochstetteri Warb. Habit: Herb. Habitat: Streamsides in wet forest, 10002750 m. Vouchers: SAJIT 004858, 005097 \& Z0014 (EA, HIB), FOKP 1303 \& 11382 (EA, HIB), Knox 3355 (EA), Mabberley 485 (EA).

Impatiens hoehnelii T.C.E. Fr. Habit: Herb. Habitat: Upper forest level, 15003470 m. Vouchers: SAJIT 006810 (EA, HIB), FOKP 10910 (EA, HIB), Hughes 2 (EA). 
Impatiens irvingii Hook. f. Habit: Herb. Habitat: Wet forest floor up to $2000 \mathrm{~m}$. Voucher: Webster 8738 (EA).

Impatiens meruensis Gilg. Habit: Herb. Habitat: Highland forest, marshes and streamside, 1000-3550 m. Vouchers: SAJIT 004780 (EA, HIB), FOKP 1720, $11385 \& 11528$ (EA, HIB), Mbuni $120 \& 264$ (EA), Lind 2828 (EA).

Impatiens meruensis subsp. cruciata (T.C.E. Fr.) Grey-Wilson. Habit: Herb. Habitat: Highland forest, marshes \& streamside, 1000-3550 m. Vouchers: SAJIT 005089 (EA, HIB), FOKP 11643 (EA, HIB), Mbuni 643 (EA), Lind 2828 (EA), Knox 3356 (EA).

Impatiens pseudoviola Gilg Habit: Herb. Habitat: Lower Highland forest, 15003300 m. Voucher: Hughes 1 (EA).

Impatiens sodenii Engl. \& Warb. Habit: Herb. Habitat: Escarpment zones with frequent mist, 1000-2700 m. Vouchers: SAJIT 004733 (EA, HIB).

Impatiens tinctoria A. Rich Habit: Herb. Habitat: Highland forest areas, streamside, 1800-3550 m. Vouchers: SAJIT 004789 \& 006819 (EA, HIB), FOKP 10919, $11356 \& 11644$ (EA, HIB), Webster 8737 (EA), Mbuni $092 \& 644$ (EA).

\section{F101. Sapotaceae}

2 Genera, 2 Species

Chrysophyllum oliviforme subsp. angustifolium (Lam.) T.D. Penn. Habit: Tree. Habitat: Moist forest, 1500-2200 m. Voucher: FOKP 1067 (EA, HIB).

Pouteria adolfi-friedericii (Engl.) A. Meeuse Habit: Tree. Habitat: Moist forest, 1500-2400 m. Vouchers: SAJIT 006904 (EA, HIB), FOKP 11004 (EA, HIB), Colby H134 (EA).

\section{F102. Ebernaceae}

2 Genera, 3 Species

Diospyros scabra (Chiov.) Cufod. Habit: Tree. Habitat: Rocky hillsides, 400-1050 m. Voucher: FOKP 11331 (EA, HIB).

Euclea divinorum Hiern Habit: Shrub. Habitat: Bushed grassland, 1-2700 m. Vouchers: FOKP 1099, $1107 \& 11461$ (EA, HIB), Mbuni $197 \& 743$ (EA).

Euclea racemos $\boldsymbol{L}$ L. Habit: Tree. Habitat: Bushland, 1-2250 m. Vouchers: FOKP 11659 (EA, HIB), Mbuni 659 (EA).

\section{F103. Primulaceae}

4 Genera, 5 Species

Anagallis hexamera P. Taylor. Habit: Herb. Habitat: streamside marshes, 22752600 m. Voucher: Hale 209 (EA).

Anagallis Serpens subsp. meyeri-johannis (Endl.) P. Taylor Habit: Herb. Habitat: Upland dry forest, 2600-3550 m. Vouchers: FOKP 988 (EA, HIB), Thulin \& Tidigs 221 (EA). 
Maesa lanceolata Forssk. Habit: Shrub. Habitat: Widespread secondary forest, Forest margin, 1300-2853 m. Vouchers: SAJIT 004770 \& 005138 (EA, HIB), FOKP 1033 (EA, HIB).

Myrsine africana L. Habit: Shrub. Habitat: Upland dry forest, 1500-3000 m. Vouchers: SAJIT 004801 (EA, HIB), FOKP 11385 (EA, HIB), Mbuni 009 \& 757 (EA), Hepper \& Field 5027 (EA), Lind \& Agnew 5160 (EA).

Rapanea melanophloeos (L.) Mez. Habit: Tree. Habitat: Upland forest, to the edge of moorland, 1200-3550 m. Vouchers: SAJIT 006806 (EA), FOKP 10906 \& 11267 (EA, HIB), Mbuni 003 (EA), Knight 87 (EA).

\section{F104. Ericaceae}

2 Genera, 6 Species

Agarista salicifolia (Lam.) G. Don Habit: Tree. Habitat: Secondary forest, high altitude bushland, forest edge, 2100-3300 m. Vouchers: SAJIT 004758 (EA, HIB), FOKP 1086 \& 1717 (EA, HIB), Friis \& Hansen 2513 (EA), Thulin \& Tidigs 257 (A).

Erica arborea L. Habit: Shrub. Habitat: Heath, woodland, Rocky, bushland, 23002700 m. Vouchers: SAJIT 004801 (EA, HIB), Thulin \& Tidigs 92 (EA).

Erica filago (Alm \& T.C.E. Fr.) Beentje Habit: Shrub. Habitat: Heath zones, 25003550 m. Voucher: Thulin \& Tidings 257 (EA).

Erica silvatica (Welw. ex Engl.) Beentje Habit: Shrub. Habitat: Moorlands, alpine zones, 2300-3550 m. Voucher: Bogdan 4984 (EA).

Erica ventricosa Thunb. Habit: Shrub. Habitat: Boggy moorlands, heath zones, 2500-3550 m. Voucher: SAJIT 004807 (EA, HIB).

Erica whyteana Britten Habit: Shrub. Habitat: Wooded grassland, 2700-3550 m. Vouchers: Thulin \& Tidings 89 (EA), Bogdan 4999 (EA).

\section{F105. Rubiaceae}

25 Genera, Species 64

Agathisanthemum globosum (Hochst. ex A. Rich.) Klotzsch Habit: Herb. Habitat: Wooded grassland, 1600-2170 m. Vouchers: Lewis 5987 (EA), Blake 2132 (EA).

Anthospermum usambarense K. Schum. Habit: Shrub. Habitat: Ericaceous belt, alpine bushland, 2150-3350 m. Voucher: Thulin \&Tidigs 251 (EA).

Anthospermum herbaceum L. f. Habit: Herb. Habitat: Forest edge, wet forest zone, 1560-2650 m. Vouchers: Mbuni 029 (EA. HIB), Symes 211 (EA).

Canthium pseudosetiflorum Bridson Habit: Shrub. Habitat: Evergreen bushland, dry woodland, 750-1750 m. Voucher: Bridson 1079 (EA).

Coffea eugenioides S. Moore Habit: Shrub. Habitat: Dry bushland, 1450-2250 m. Voucher: Thomas 232 (EA).

Conostomium quadrangulare (Rendle) Cufod. Habit: Herb. Habitat: Rocky bushland, 60-1950 m. Voucher: Napier 2067 (EA).

Fadogia cienkowskii Schweinf. Habit: Shrub. Habitat: Wooded grassland, 18001950 m. Voucher: Napier 2005 (EA). 
Fagaropsis angolensis (Engl.) H.M. Gardner Habit: Tree. Habitat: Dry Forest, 1150-2100 m. Voucher: Lindsay 16 (EA).

Galiniera coffeoides Delile Habit: Shrub. Habitat: Moist forest, 1850-2400 m. Vouchers: Friis \& Hansen 2552 (EA), Dale 907(EA).

Galiniera saxifraga (Hochst.) Bridson Habit: Shrub. Habitat: Moist forest, 18502400 m. Vouchers: SAJIT 005072 \& 006795 (EA, HIB), FOKP 936, 1074, 10895, $11361 \& 11412$ (EA, HIB), Mbuni 148 (EA).

Galium aparine L. Habit: Climber. Habitat: Grassland, 1600-3200 m. Voucher: SAJIT 006856 (EA, HIB).

Galium aparinoides Forssk. Habit: Climber. Habitat: Montane forest edges, 16003500 m. Vouchers: FOKP 11404 (EA, HIB), Mbuni 030 (EA), Verdcourt \& Taylor et al. 2434 (EA).

Galium scioanum Chiov. Habit: Herb. Habitat: Moist woodland, 1800-2700 m. Vouchers: FOKP 1138 \& 11536 (EA, HIB), Mbuni 272 (EA), Thulin \& Tidings 175 (EA), Tweedie 2699 (EA).

Galium kenyanum Verdc. Habit: Herb. Habitat: Alpine grassland, 2880-3540 m. Voucher: Tweedie 3864 (EA), Mabberley 51 (EA).

Galium ruwenzoriense (Cortesi) Ehrend. Habit: Herb. Habitat: Forest edges \& bushland, 2700-3500 m. Vouchers: Knox 3387 (EA), Thulin \& Tidigs 101 (EA).

Galium thunbergianum Eckl. \& Zeyh. Habit: Herb. Habitat: Montane forest, 2050-3550 m. Vouchers: SAJIT 006857 (EA, HIB), FOKP 1060 (EA, HIB), Knox 3376 (EA).

Gardenia ternifolia Schumach. \& Thonn. Habit: Tree. Habitat: Wooded grassland, riverine, 1100-2500 m. Voucher: Champion 189 (EA).

Gardenia volkensii K. Schum. Habit: Shrub. Habitat: Riverine woodland, wooded grassland, 1-1750 m. Vouchers: SAJIT 006878 (EA, HIB), FOKP 11310 (EA, HIB), Rindsay 1958 (EA).

Keetia gueinzii (Sond.) Bridson. Habit: Climber. Habitat: Moist forest, bushland, riverine, 1300-2500 m. Vouchers: SAJIT 004819 \& 005066 (EA, HIB), FOKP 1017 (EA, HIB), Mbuni 052 (EA, HIB), Mabberley 155 (EA), Lind 2849 (EA), Friis \& Hansen 2545 (EA).

Kohautia coccinea Royle Habit: Shrub. Habitat: Riverine woodland, rocky bushland, dry grassland, 1250-2500 m. Vouchers: Faden 144 (EA), Symes 208 (EA).

Meyna tetraphylla (Schweinf. ex Hiern) Robyns. Habit: Shrub. Habitat: Riverine woodland, rocky bushland, 1-1400 m. Voucher: Faden $77 \& 792$ (EA).

Mitracarpus villosus (Sw.) DC. Habit: Herb. Habitat: As weed on cultivated ground, 60-1400 m. Voucher: SAJIT Z0008 (HIB).

Oldenlandia corymbosa var. nana (Bremek.) Verdc. Habit: Herb. Habitat: Dry areas, 1-2500 m. Voucher: Symes 754 (EA).

Oldenlandia corymbosa var. caespitosa (Benth). Habit: Herb. Habitat: Dry areas, 1-2500 m. Voucher: Verdcourt 736 (EA).

Oldenlandia herbacea (L.) Roxb. Habit: Herb. Habitat: Dry grassland, 75-2400 m. Vouchers: FOKP 11315 (EA, HIB), Mbuni 051 (EA). 
Oldenlandia lancifolia (Schumach.) DC. Habit: Herb. Habitat: Wooded grassland, 400-2350 m. Voucher: SAJIT 007055 (EA, HIB).

Oldenlandia monanthos (Hochst. ex A. Rich.) Hiern: Habit: Herb. Habitat: Montane grassland, 1650-3150 m. Vouchers: SAJIT 006820 (EA, HIB), FOKP 10920 (EA, HIB).

Oxyanthus speciosus DC. Habit: Tree. Habitat: Forest riverine, 1400-2200 m. Vouchers: SAJIT 006898 (EA, HIB), FOKP 1275, 1736 \& 11463 (EA, HIB), Mbuni 199 (EA).

Oxyanthus speciosus subsp. stenocarpus (K. Schum.) Bridson Habit: Tree. Habitat: Riverine forest, 1400-2200 m. Voucher: SAJIT 007095 (EA, HIB).

Pavetta abyssinica Fresen. Habit: Shrub. Habitat: Forest riverine, secondary bushland, 1500-2550 m. Vouchers: SAJIT 005069 (EA, HIB), FOKP 1084, 1719 \& 11607 (EA, HIB), Mbuni 607 (EA).

Pavetta crassipes K. Sch. Habit: Shrub. Habitat: Wooded grassland, 900-1500 m. Voucher: Nyamwaya 28 (EA).

Pavetta dolichantha Bremek. Habit: Shrub. Habitat: Bushland and rocky slopes, 750-1650 m. Voucher: Tweedie 3029 (EA).

Pavetta elliottii K. Schum. \& K. Krause. Habit: Shrub. Habitat: Bushland, grassland, forest margin, 1400-1900 m. Voucher: Pole et al. 1512 (EA).

Pavetta gardeniifolia Hochst. ex A. Rich. Habit: Shrub. Habitat: Rocky, bushland, 7005-2500 m. Vouchers: FOKP 11429 (EA, HIB), Mbuni 165 (EA).

Pentas decora S. Moore. Habit: Herb. Habitat: Wooded grassland, 1590-2700 m. Voucher: Lucas 200 (EA).

Pentas lanceolata (Forssk.) Deflers. Habit: Shrub. Habitat: Forest edge, 12002830 m. Voucher: Paswa 49 (EA).

Pentas lanceolata var. leucaster (K. Krause) Verdc. Habit: Herb. Habitat: Forest edges, 1200-2830 m. Vouchers: Napier 1902 (EA), Lind 5093 (EA).

Pentas longiflora Oliv. Habit: Herb. Habitat: Bushed grassland, 500-2500 m. Vouchers: FOKP 1095, 11299 \& 11621 (EA, HIB), Mbuni 621 (EA).

Pentas parvifolia Hiern. Habit: Shrub. Habitat: Bushed grassland, 650-2400 m. Vouchers: FOKP 11467 (EA, HIB), Mbuni 203 (EA).

Pentas pubiflora S. Moore Habit: Shrub. Habitat: Montane and riverine forest, 1800-2400 m. Vouchers: FOKP 11290 (EA, HIB), Mbuni 026 (EA).

Pentas zanzibarica (Klotzsch) Vatke Habit: Shrub. Habitat: Riverine forest, 18002440 m. Vouchers: FOKP 11620 (EA, HIB), Mbuni 620 (EA).

Pentas schimperiana Vatke Habit: Herb. Habitat: Forest clearing, 2100-2700 m. Vouchers: SAJIT 004741 (EA, HIB), FOKP 1008 (EA, HIB), Tweedie 2801 (EA), Gardener 2852 (EA).

Pentanisia ouranogyne S. Moore. Habit: Herb. Habitat: Grassland, 300-2400 m. Voucher: Thomasson \& Hansson 91A (EA).

Pentanisia schweinfurthii Hiern. Habit: Herb. Habitat: Wooded grassland, 15002480 m. Voucher: Symes 278 (EA). 
Psychotria fractinervata E.M.A. Petit Habit: Shrub. Habitat: Ocotea forest, 19002550 m. Voucher: FOKP 1873 (EA, HIB).

Psychotria kirkii Hiern. Habit: Shrub. Habitat: Bushland, Wooded grassland, 2502250 m. Vouchers: FOKP 11298 \& 11437 (EA, HIB), Mbuni 173 (EA).

Psychotria mahonii var. puberula (E.M.A. Petit) Verdc. Habit: Tree. Habitat: Swampy forest, riverine, 1400-2400 m. Voucher: Gardener 1398a (EA).

Psychotria mahonii var. pubescens (Robyns) Verdc. Habit: Tree. Habitat: Swampy forest, riverine, 1400-2400 m. Voucher: Tweedie 2801 (EA).

Pyrex schimperiana (A. Rich.) Bridson Habit: Tree. Habitat: Riverine, 14002400 m. Vouchers: FOKP 11474 (EA, HIB), Mbuni 210 (EA).

Richardia brasiliensis Gomes Habit: Herb. Habitat: Grassland, 1500-2010 m. Voucher: FOKP 11489 (EA, HIB), Mbuni 225 (EA).

Rubia cordifolia L. Habit: Herb. Habitat: Woodland clearings, riverine bushland, 1240-3120 m. Voucher: Mungai 84 \& 128 (EA).

Rutidea orientalis Bridson Habit: Shrub. Habitat: Woodland, 1400-2400 m. Vouchers: SAJIT 006891 (EA, HIB), FOKP 10991 (EA, HIB).

Rytigynia neglecta (Hiern) Robyns Habit: Tree. Habitat: Riverine forest, 1350 2200 m. Vouchers: FOKP 1809 (EA, HIB), Paswa 54 (EA).

Rytigynia ubligii (K. Schum. \& K. Krause) Verdc. Habit: Tree. Habitat: Wet forest, 1050-2400 m. Vouchers: FOKP 11404, 11452 \& 11603 (EA, HIB), Mbuni 140, $188 \& 603$ (EA, HIB).

Spermacoce minutiflora (K. Schum.) Verdc. Habit: Herb. Habitat: Woodland, grassland, 1500-2350 m. Voucher: Napier 1973 (EA).

Spermacoce princeae (K. Schum.) Verdc. Habit: Herb. Habitat: Wet forest, 1470 2650 m. Vouchers: SAJIT 006926 (EA, HIB), FOKP 1129 (EA, HIB).

Spermacoce sphaerostigma (A. Rich.) Habit: Herb. Habitat: Grassland, 900-2200 m. Voucher: Symes 198 \& 756 (EA).

Coptosperma graveolens (S. Moore) Bremek. Habit: Tree. Habitat: Bushland, forest thicket, 1-2500 m. Vouchers: FOKP 11429 (EA, HIB), Mbuni 165 (EA).

Coptosperma pavettoides (Harv.) Sim Habit: Shrub. Habitat: Secondary bushland, 1200-1750 m. Voucher: FOKP 1009 (EA, HIB).

Vangueria apiculata K. Schum. Habit: Tree. Habitat: Thickets, riverine, wooded grassland, bushland, 950-2300 m. Vouchers: SAJIT 004742 \& 005061 (EA, HIB), Nyamwaya 31 (EA).

Vangueria infausta Burch. Habit: Shrub. Habitat: Thickets, riverine, wooded grassland, bushland, 1-2100 m. Vouchers: SAJIT 006853 (EA, HIB), FOKP 10953 (EA, HIB).

Vangueria linearisepala K. Schum. Habit: Shrub. Habitat: Wooded grassland, bushland, 750-2100 m. Voucher: Friis \& Hansen 2539 (EA).

Vangueria madagascariensis J.F. Gmel. Habit: Tree. Habitat: Riverine, wooded grassland, bushland, 750-2100 m. Vouchers: Mbuni 751 (EA), Maundu \& Musya 31 (EA).

Vangueria volkensii K. Schum. Habit: Tree. Habitat: Riverine, bushland, 9002300 m. Vouchers: FOKP 956, 1023, 1075 \& 11405 (EA, HIB), Mbuni 141, 738 (EA), Bridson 105 (EA). 


\section{F106. Gentianaceae}

3 Genera, 11 Species

Chironia elgonensis Bullock. Habit: Herb. Habitat: Swamps, 1705-2325 m. Voucher: Symes 1406 (EA).

Sebaea brachyphylla Griseb. Habit: Herb. Habitat: Wooded grassland, 16503470 m. Vouchers: Thulin 218 (EA), Verdcourt 2439 (EA).

Sebaea grandis (E. Mey.) Steud. Habit: Herb. Habitat: Wooded grassland on shallow soil, 1550-2160 m. Voucher: Symes 1010 (EA).

Swertia abyssinica Hochst. Habit: Herb. Habitat: Alpine zone, 2000-3300 m. Vouchers: Mabberley 580 (EA), Nemomissa 970830 (EA).

Swertia brownii J. Shah. Habit: Herb. Habitat: Wooded grassland, 1800-2600 m. Vouchers: SAJIT 006925 (EA, HIB), FOKP 1128 (EA, HIB).

Swertia crassiuscula Gilg Habit: Herb. Habitat: Wet stony, heath, lower alpine zones, 2700-3500 m. Vouchers: Dale 3263 \& 3264 (EA).

Swertia kilimandscharica Engl. Habit: Herb. Habitat: Montane, subalpine, 21003550 m. Vouchers: FOKP 11282 (EA), Mbuni 018 (EA), Mabberley \& McCall 124 (EA), Lucas 169 (EA), Mainwaring 46 (EA).

Swertia lugardiae Bullock Habit: Herb. Habitat: Montane and subalpine grassland, 2100-3500 m. Vouchers: Sileshi 4 \& 5 (EA).

Swertia tetrandra Hochst. Habit: Herb. Habitat: Disturbed upland grassland, 18002200 m. Voucher: SAJIT 005101 (EA, HIB).

Swertia usambarensis Engl. Habit: Herb. Habitat: Montane grassland, 16003500 m. Vouchers: Mabberley \& McCall 264 (EA), Thulin \& Tidigs 39 (EA), Symes 623 (EA).

Swertia welwitschii Engl. Habit: Herb. Habitat: Roadside, 1845-2400 m. Voucher: Jex-Blake 2122 (EA).

\section{F107. Loganiaceae}

1 Genus, 2 Species

Strychnos henningsii Gilg. Habit: Tree. Habitat: Forest thicket on rocky hills, 8502100 m. Vouchers: SAJIT Z0035 (HIB), FOKP 11661 (EA), Mbuni 661(EA).

Strychnos mitis S. Moore Habit: Tree. Habitat: Riverine forest, 1-1950 m. Vouchers: FOKP 11464 (EA), Mbuni 200 (EA).

\section{F108. Apocynaceae}

20 Genera, 37 Species

Acokanthera oppositifolia (Lam.) Codd Habit: Shrub. Habitat: Woodland, 14502100 m. Voucher: FOKP 1097 (EA, HIB).

Acokanthera schimperi (A. DC.) Schweinf. Habit: Tree. Habitat: Wooded grassland, 1200-2300 m. Voucher: FOKP 11306 (EA, HIB). 
Caralluma arachnoidea (P.R.O. Bally) M.G. Gilbert. Habit: Herb. Habitat: Rocky grassland, 100-1300 m. Voucher: Hartmann \& Newton 284046 (EA).

Caralluma dicapuae (Chiov.) Chiov. Habit: Herb. Habitat: Rocky bushland, 7001700 m. Voucher: FOKP 1260 (EA, HIB).

Caralluma peckii P.R.O. Bally Habit: Herb. Habitat: Rare in rocky bushland, 9001150 m. Voucher: Bally 12359 (EA).

Carissa edulis (Forssk.) Vahl Habit: Shrub. Habitat: Bushland, thicket, grassland, 1-2550 m. Vouchers: FOKP 11681 (EA), Mbuni 681 (EA).

Carissa spinarum L. Habit: Shrub. Habitat: Forest edges woodland, 1-2550 m. Vouchers: FOKP 1109, 1272 \& 11439 (EA, HIB), Mbuni 175 (EA).

Ceropegia ballyana Bullock Habit: Climber. Habitat: Dry bushland, 900-1400 m. Voucher: Masinde 683 (EA).

Ceropegia racemosa N.E. Br. Habit: Climber. Habitat: Evergreen forest, 1-1900 m. Voucher: FOKP 1822 (EA, HIB).

Gomphocarpus fruticosus subsp. flavidus (N.E. Br.) Goyder Habit: Shrub. Habitat: Upland grassland, 900-2900 m. Vouchers: Napier 2039 (EA), Hepper 5056 (EA).

Gomphocarpus phillipsiae (N.E. Br.) Goyder Habit: Shrub. Habitat: Upland grassland, 1600-3050 m. Vouchers: FOKP 11279 (EA, HIB), Mbuni 015 (EA).

Gomphocarpus physocarpus E. Mey. Habit: Shrub. Habitat: Upland grassland, 1800-3000m. Vouchers: SAJIT 004771 (EA, HIB), FOKP 11300 (EA, HIB), Mbuni 056 (EA, HIB).

Gomphocarpus stenoglossus Schltr. Habit: Herb Habitat: Grassland, rocky soils, 1600-3050 m. Voucher: FOKP 1817 (EA, HIB).

Landolphia buchananii (Hallier f.) Stapf Habit: Climber. Habitat: Riverine, 13502150 m. Vouchers: FOKP 1270 \& 11273 (EA, HIB).

Leptadenia lancifolia (Schumach. \& Thonn.) Decne. Habit: Climber. Habitat: Alluvial \& Riverine bushland, 1650-2600 m. Voucher: Balley 12360 (EA).

Margaretta rosea Oliv. Habit: Herb. Habitat: Grasslands, 1600-2500 m. Voucher: Symes 280 (EA).

Marsdenia robusta Balf. f Habit: Climber. Habitat: bushland, 1600-2000 m. Voucher: FOKP 11310 (EA, HIB).

Marsdenia angolensis N.E. Br. Habit: Climber. Habitat: Shrubland and Riverine bushland, 1600-2000 m. Voucher: Tweedie 2403 (EA).

Marsdenia rubicunda N.E. Br. Habit: Climber. Habitat: Riverine, bushland in dry areas, 0-1300 m. Voucher: Goyder \& Masinde 3952 (EA).

Marsdenia schimperi Decne. Habit: Climber. Habitat: Upland forest edge, 18002650 m. Vouchers: SAJIT 006886 (EA, HIB), FOKP 1884 \& 10986 (EA, HIB).

Mondia whitei (Hook. f.) Skeels. Habit: Climber. Habitat: 1600-2000 m. Voucher: FOKP 998 (EA, HIB).

Pachycarpus eximius (Schltr.) Bullock. Habit: Herb. Habitat: Uncommon in grassland, 1600-2500 m. Voucher: Haper \& Field 5038 (EA).

Pachycarpus grantii (Oliv.) Bullock Habit: Herb. Habitat: Montane grassland, 1850-2950 m. Voucher: Hepper et al. 5038 (EA). 
Pachycarpus lineolatus (Decne.) Bullock Habit: Herb. Habitat: Dry wooded grassland, 1000-2700 m. Voucher: Napier 2019 (EA).

Pachycarpus petherickianus (Oliv.) Goyder. Habit: Herb. Habitat: 1000-2000 m. Voucher: Tweedie 380 (EA).

Pentarrbinum gonoloboides (Schltr.) Liede Habit: Climber. Habitat: Montane forest, 2500-3200 m. Voucher: Tweedie 3912 (EA).

Pergularia daemia (Forssk.) Chiov. Habit: Herb. Habitat: Upland forest, around 2050 m. Vouchers: FOKP 1268 \& 11481 (EA, HIB), Mbuni $217 \& 749$ (EA).

Periploca linearifolia Quart.-Dill. \& A. Rich. Habit: Climber. Habitat: Upland forest, 1900-2900 m. Vouchers: SAJIT 005059 (EA, HIB), FOKP 1076 (EA, HIB), Mbuni 044 \& 622 (EA, HIB), Bridson 104 (EA), Fattan 13876 (EA).

Saba comorensis (Bojer ex A. DC.) Pichon. Habit: Shrub. Habitat: Riverine forest, 1-1800 m. Voucher: Mabberley \& McCall 98 (EA).

Secamone attenuifolia Goyder Habit: Shrub. Habitat: Bushland, wooded grassland, 1000-1850 m. Voucher: Tweedie 3638 (EA).

Stathmostelma pedunculatum (Decne.) K. Schum. Habit: Herb. Habitat: Sandy grassland, 0-2300 m. Voucher: Lucas 190 (EA).

Tabernaemontana stapfiana Britten Habit: Tree. Habitat: Wet forest, 1500-2300 m. Vouchers: SAJIT 006887 (EA, HIB), FOKP 1112, 10987 (EA, HIB).

Tylophora heterophylla A. Rich. Habit: Climber. Habitat: Upper forest, 2600-3500 m. Vouchers: FOKP 11600 (EA), Mbuni 596 \& 600 (EA), Mabberley \& McCall 65 (EA). Tylophora lugardae Bullock Habit: Climber. Habitat: Upland forest, 2000-2500 m. Voucher: Archer 303 (EA).

Tylophora tenuipedunculata K. Schum. Habit: Climber. Habitat: Upland forest up to 1500 m. Vouchers: FOKP 11453 (EA, HIB), Mbuni 189 (EA).

Xysmalobium heudelotianum Decne. Habit: Herb. Habitat: Dry grassland, 15002000 m. Voucher: Napier 1974 (EA).

\section{F109. Boraginaceae}

6 Genus, 11 Species

Cordia africana Lam. Habit: Tree. Habitat: Riverine, forest, wooded grassland, 1050-2100 m. Voucher: SAJIT 005062 (EA, HIB).

Cynoglossum amplifolium Hochst. ex A. DC. Habit: Herb. Habitat: Montane forest, 2100-3430 m. Voucher: Irwin 423 (EA).

Cynoglossum aequinoctiale T.C.E. Fr. Habit: Herb. Habitat: Montane grassland, 1770-2480 m. Voucher: Thulin \& Tidigs 77 (EA).

Cynoglossum cheranganiense Verdc. Habit: Herb. Habitat: Rare in alpine grassland, heath and upper forest zone, 2480-3255 m. Vouchers: Tweedie 2984 (EA), Mabberley 207 (EA), Lucas 168 (EA).

Cynoglossum coeruleum Hochst. ex A. DC. Habit: Herb. Habitat: Montane forest, 1100-3200 m. Voucher: FOKP 1245 \& 11435 (EA, HIB), Mbuni 171 (EA), Dale 15331 (EA). 
Ehretia cymosa Thonn. Habit: Shrub. Habitat: Evergreen forest, dry or wet forest patches, 1050-2300 m. Vouchers: FOKP 1882 \& 11625 (EA, HIB), Mbuni 625 (EA).

Lithospermum afromontanum Weim. Habit: Shrub. Habitat: Montane forest edges, heath woodland, 2100-3350 m. Vouchers: Tweedie 3828 (EA), Thulin \& Tidigs 239 (EA).

Myosotis abyssinica Boiss. \& Reut. Habit: Herb. Habitat: Montane forest edges, heath woodland, 1560-3590 m. Voucher: Thulin \& Tidings 236 (EA).

Myosotis keniensis T.C.E. Fr. Habit: Herb. Habitat: Alpine zones, 3150-3350 m. Voucher: Thulin \& Tidigs 236 (EA).

Trichodesma physaloides (Fenzl) A. DC. Habit: Herb. Habitat: Wooded grassland, 700-2400 m. Voucher: Mortimer 275 (EA).

Trichodesma zeylanicum (Burm. f.) R. Br. Habit: Herb. Habitat: Dry bushland, 250-1710 m. Vouchers: FOKP 11281 (EA), Mbuni 017 (EA).

\section{F110. Convolvulaceae}

7 Genera, 15 Species

Astripomoea lachnosperma (Choisy) A. Meeuse Habit: Herb. Habitat: Bushland, 750-2600 m. Voucher: Napier 2035 (EA, HIB).

Astripomoea malvacea (Klotzsch) A. Meeuse. Habit: Shrub. Habitat: Wooded grassland, 725-2240 m. Voucher: Napier 2074 (EA).

Convolvulus kilimandschari Engl. Habit: Climber. Habitat: Bamboo thicket, 24003500 m. Voucher: FOKP 1062 (EA, HIB).

Convolvulus wightii Wall. Habit: Herb. Habitat: Grassland, 1500-2500 m. Voucher: FOKP 1292 (EA, HIB).

Cuscuta kilimanjari Oliv. Habit: Climber. Habitat: Common in forest areas, 15002770 m. Voucher: SAJIT 006901 (EA, HIB).

Cuscuta epilinum Weihe Habit: Climber. Habitat: Cultivated ground, 1800-2250 m. Voucher: Schouten 58 \& H348 (EA).

Cuscuta planiflora Ten. Habit: Climber. Habitat: Occurring on various hosts, 1500 3000 m. Vouchers: FOKP 11553 (EA, HIB), Mbuni 290 (EA).

Ipomoea cairica (L.) Sweet. Habit: Herb. Habitat: Swampy grassland, 6000-1950 m. Voucher: SAJIT Z0056 (EA).

Ipomoea tenuirostris Choisy Habit: Herb. Habitat: Bushland and thicket, 16502710 m. Vouchers: FOKP 1885 \& 11510 (EA, HIB), Mbuni 246 (EA), Tweedie 3115 (EA), Webster 8924 (EA).

Ipomoea crepidiformis Hallier f. Habit: Herb. Habitat: Wooded grassland, 9002150 m. Voucher: Champion T3 (EA).

Ipomoea spathulata Hallier f. Habit: Shrub. Habitat: Acacia-Commiphora Bushland, 610-1640 m. Vouchers: FOKP 11440 (EA) \& Mbuni 176 (EA).

Ipomoea kituiensis Vatke. Habit: Herb. Habitat: Dry bushland, 180-1860 m. Vouchers: SAJIT 006850 (EA, HIB), FOKP 11510 \& 10950 (EA, HIB), Mbuni 246 (EA). Ipomoea tenuirostris Choisy Habit: Herb. Habitat: Bushland and thicket, 16502710 m. Voucher: Tweedie 3115 (EA). 
Stictocardia beraviensis (Vatke) Hallier f. Habit: Shrub. Habitat: Bushland, 9001800 m. Voucher: Honore 3451 (EA).

Turbina stenosiphon (Hallier f.) A. Meeuse Habit: Climber. Habitat: Bushland and in rocky areas, 900-1800 m. Voucher: Tweedie 1926 (EA).

\section{F111. Solanaceae}

5 Genera, 18 Species

Cestrum aurantiacum Lindl. Habit: Shrub. Habitat: Cultivated and disturbed ground, 1000-2500 m. Vouchers: FOKP 1020 (EA, HIB), Mbuni 085 (EA, HIB).

Discopodium penninervium Hochst. Habit: Shrub. Habitat: Bamboo zone, 29003300 m. Voucher: FOKP 1891 (EA, HIB).

Physalis lagascae Roem. \& Schult. Habit: Herb. Habitat: Cultivated and disturbed ground, 40-1850 m. Voucher: Meyerhoff 12 (EA).

Physalis peruviana L. Habit: Herb. Habitat: Cultivated and naturalized, 1700 2480 m. Voucher: Mabberley \& McCall 108 (EA).

Solanum aculeastrum Dunal. Habit: Shrub. Habitat: Forest margin, 1800-2650 m. Vouchers: FOKP 935 \& 11364 (EA, HIB), Mbuni 100 (EA), Symes 635 (EA).

Solanum aculeatissimum Jacq. Habit: Herb. Habitat: Montane forest, 1425-2640 m. Voucher: FOKP 11397 (EA), Mbuni 133 (EA), Hepper \& Field 4985 (EA).

Solanum anguivi Lam. Habit: Shrub. Habitat: Cultivated, 1230-2700 m. Vouchers: SAJIT 005143 (EA, HIB), FOKP 966 \& 11535 (EA, HIB), Mbuni 271 (EA), Hepper \& Field 4986 (EA).

Solanum betaceum Cav. Habit: Shrub. Habitat: Cultivated, 1200-2800 m. Voucher: FOKP 1879 (EA, HIB).

Solanum campylacanthum Hochst. Habit: Shrub. Habitat: Cultivated, 10302000 m. Vouchers: FOKP 1296 \& 11480 (EA, HIB), Mbuni 216 (EA).

Solanum giganteum Jacq. Habit: Shrub. Habitat: Forest margin, Undergrowth, 1500-2400 m. Vouchers: FOKP 11365 (EA, HIB) \& Mbuni 100 (EA).

Solanum hastifolium Dunal Habit: Shrub. Habitat: Dry bushland or grassland, 501400 m. Voucher: Carter \& Stannard 59 (EA).

Solanum mauense Bitter Habit: Shrub. Habitat: Secondary forest, 1800-2700 m. Vouchers: SAJIT 004754 \& 004774 (EA, HIB), FOKP 11291 (EA, HIB), Mbuni 027 (EA).

Solanum mauritianum Scop. Habit: Tree. Habitat: Cultivation, 1450-2750 m. Vouchers: FOKP 1069 \& 11397 (EA, HIB), Mbuni 133 (EA).

Solanum nakurense C.H. Wright. Habit: Shrub. Habitat: Upland bushland, 9002950 m. Vouchers: FOKP 1006 \& 11605 (EA, HIB), Mbuni 605 (EA), Hepper \& Field 4976 (EA), Webster 8918 (EA).

Solanum nigrum L. Habit: Shrub. Habitat: Cultivated, 0-3070 m. Vouchers: FOKP 11341 (EA, HIB), Mbuni 077 (EA), Webster 8918 (EA).

Solanum sessilistellatum Bitter. Habit: Shrub. Habitat: Montane rain forest, 2130 2990 m. Vouchers: FOKP 11379 (EA, HIB), Mbuni 115 (EA), Jackson 2424 (EA). 
Solanum terminale Forssk. Habit: Shrub. Habitat: Riverine, thickets, 1350-2950 m. Vouchers: SAJIT 004736 (EA, HIB), FOKP 1888, 11297 (EA, HIB), Mbuni 033 (EA), Irwin 139 (EA).

Withania somnifera (L.) Dunal. Habit: Shrub. Habitat: Forest margin, 450-2250 m. Vouchers: FOKP 11384 \& 14477 (EA, HIB), Mbuni 119 \& 213 (EA).

\section{F112. Oleaceae}

2 Genera, 5 Species

Jasminum abyssinicum Hochst. ex DC. Habit: Climber. Habitat: Upland forest edges, bushland, open and riverine, 900-3000 m. Vouchers: SAJIT 005088 (EA, HIB), FOKP 11353 (EA, HIB), Webster 8823 (EA).

Jasminum fluminense Vell. Habit: Climber. Habitat: Dry Combretum, Albizia woods, 280-2700 m. Vouchers: SAJIT 006807 (EA, HIB), FOKP 10907 (EA, HIB).

Olea europaea subsp. africana (Mill.) P.S. Green. Habit: Tree. Habitat: Upland forest, 1150-2550 m. Voucher: SAJIT 004756 (EA, HIB).

Olea europaea subsp. cuspidata (Wall. \& G. Don) Cif. Habit: Tree. Habitat: Upland evergreen forest, 950-2400 m. Voucher: FOKP 1798 (EA, HIB).

Olea africana Mill. Habit: Tree. Habitat: Upland forest, 1150-2550 m. Voucher: Lind 5088 (EA).

\section{F113. Plantaginaceae}

4 Genera, 5 Species

Callitriche oreophila Schotsman. Habit: Herb. Habitat: Bamboo zone, 19003500 m. Voucher: Townsend 2389 (EA).

Plantago palmata Hook. f. Habit: Herb. Habitat: Montane rain forest, Path sides, 1704-3550 m. Vouchers: FOKP 1065 \& 11552 (EA, HIB), Mbuni 289 (EA), Pudwa 67 (EA).

Plantago lanceolata L. Habit: Herb. Habitat: Path sides, 1650-2750 m. Voucher: Tweedie 2806 (EA).

Sibthorpia europaea L. Habit: Herb. Habitat: Alpine zone, 1860-2345 m. Voucher: Mabberley 576 (EA).

Veronica abyssinica Fresen. Habit: Herb. Habitat: Upland grassland, woodland edges, 1560-3500 m. Voucher: Symes 516 (EA).

\section{F114. Scrophulariaceae}

7 Genera, 7 Species

Alectra asperrima Benth. Habit: Herb. Habitat: Montane wet grassland, 20003000 m. Voucher: Thorold 2761 (EA). 
Buddleja polystachya Fresen. Habit: Tree. Habitat: Montane forest, Bushland, 1000-2700 m. Vouchers: SAJIT 006895 (EA, HIB), FOKP 965, 11319, 11327 \& 11393 (EA, HIB), Mbuni 055 \& 128 (EA).

Cycnium tenuisectum (Standl.) O.J. Hansen Habit: Tree. Habitat: Bushland, 1000$2700 \mathrm{~m}$. Voucher: SAJIT 007119 (EA, HIB).

Hebenstretia dentata L. Habit: Shrub. Habitat: Grassland at around $2300 \mathrm{~m}$. Voucher: Thulin 119 (EA).

Limosella africana Glück Habit: Herb. Habitat: Upland grassland, 1600-3500 m. Voucher: Mabberley 570 (EA).

Selago thomsonii Rolfe. Habit: Shrub. Habitat: Dry subalpine heathland, 18603380 m. Vouchers: Dale 3395 (EA), Part II Botany 5159 (EA).

Verbascum scrophulariifolium (Hochst.) Hub.-Mor. Habit: Herb. Habitat: Upland grassland, 1950-2635 m. Voucher: Thulin \& Tidigs 44 (EA).

\section{F115. Stilbaceae}

3 Genera, 4 Species

Halleria lucida L. Habit: Tree. Habitat: Montane forest, Drier forests associated with cedar, podo and Bamboo, 1550-2750 m. Vouchers: SAJIT 005142 \& 006802 (EA, HIB), FOKP 1014, 10902 \& 11354 (EA, HIB), Mbuni 619 (EA), Agnew et al. 5076 (EA).

Hebenstretia angolensis Rolfe. Habit: Shrub. Habitat: Rocky heathlands, 15003500 m. Vouchers: Mbuni 125 (EA), Bogdan 4390 (EA).

Nuxia congesta R. Br. ex Fresen. Habit: Tree. Habitat: Montane forest, bamboo Zone, 1550-2850 m. Vouchers: SAJIT 005068 (EA, HIB), FOKP $11350 \& 11370$ (EA, HIB).

Nuxia oppositifolia (Hochst.) Benth. Habit: Tree. Habitat: Riverine forest, bushland, 1550-2850 m. Voucher: Honare 3716 (EA).

\section{F116. Linderniaceae}

3 Genera, 7 Species

Craterostigma plantagineum Hochst. Habit: Herb. Habitat: Over rocks, 11602300 m. Voucher: Hepper \& Field 5063 (EA).

Craterostigma pumilum Hochst. Habit: Herb. Habitat: Over rocks, in montane, 1800-3300 m. Voucher: Hepper \& Field 5026 (EA).

Cycnium adonense E. Mey. ex Benth. Habit: Herb. Habitat: Wooded grassland, 0-2550 m. Vouchers: Lucas 194 (EA), Symes 32 (EA).

Lindernia abyssinica Engl. Habit: Herb. Habitat: Shallow soil, 1800-2600 m. Voucher: Maas 4744 (EA).

Lindernia pulchella (Skan) Philcox Habit: Herb. Habitat: Short grassland, 400$1800 \mathrm{~m}$. Voucher: Tweedie 3810 (EA). 
Lindernia rotundifolia (L.) Alston Habit: Herb. Habitat: Grassy marshes, 16501900 m. Vouchers: FOKP 1146, 1236 \& 1795 (EA, HIB).

Lindernia schweinfurthii (Engl.) Dandy Habit: Herb. Habitat: Shallow soils, 17001850 m. Voucher: Lucas 174 (EA).

\section{F117. Pedaliaceae}

1 Genus, 2 Species

Sesamum angolense Welw. Habit: Shrub. Habitat: Grassland and roadside, 4002400 m. Voucher: FOKP 1039 (EA, HIB).

Sesamum calycinum Welw. Habit: Herb. Habitat: Grassland and wasteland, 3101340 m. Voucher: FOKP 1039 (EA, HIB).

\section{F118. Lamiaceae}

32 Genera, 68 Species

Achyrospermum schimperi (Hochst. ex Briq.) Perkins Habit: Shrub. Habitat: Wet streamside, 1600-3000 m. Vouchers: FOKP 11311 (EA), Mbuni 047 (EA).

Ajuga remota Benth. Habit: Herb. Habitat: Montane forest, 1500-3150 m. Vouchers: FOKP 11473 (EA, HIB), Mbuni 209 (EA).

Aeollanthus densiflorus Ryding Habit: Herb. Habitat: wooded grassland, Rocky slopes 1730-2300 m. Voucher: FOKP 1813 (EA, HIB).

Aeollanthus repens Oliv. Habit: Shrub. Habitat: Wooded grassland, 1550-2700 m. Vouchers: FOKP 1031, Thulin \& Tidigs 196 (EA).

Becium decumbens (Gürke) A.J. Paton Habit: Herb. Habitat: grassland, 15503200 m. Voucher: Townsend 2355 (EA).

Bridson rotundifolium (Briq.) A.J. Paton Habit: Herb. Habitat: Wooded grassland, 1500-2800 m. Voucher: Bridson 102 (EA).

Clerodendrum capense Eckl. \& Zeyh. ex Schauer Habit: Shrub. Habitat: Wooded grassland, 1200-1900 m. Voucher: SAJIT 005081 (EA, HIB).

Clerodendrum johnstonii Oliv. Habit: Shrub. Habitat: Wet forest margin, 12002500 m. Vouchers: SAJIT 004723, $005081 \& 006817$ (EA, HIB), FOKP 10917 \& 11432 (EA, HIB), Mbuni 168 \& 739 (EA, HIB), Tweedie 4201 (EA).

Clerodendrum umbellatum Poir. Habit: Herb. Habitat: Wooded grassland, up to 1860 m. Vouchers: Napier 2007 (EA), Tweedie 2732 (EA).

Clinopodium abyssinicum (Benth.) Kuntze Habit: Herb. Habitat: Woodland, 1000-3000 m. Voucher: Verdcourt 2439 (EA).

Fuerstia africana T.C.E. Fr. Habit: Herb. Habitat: Grassland, 800-2400 m. Vouchers: FOKP 11295, 11431 \& 11728 (EA, HIB), Mbuni 031, $167 \& 728$ (EA).

Geniosporum paludosum Baker. Habit: Herb. Habitat: Grassland, 1000-2500 m. Voucher: Ivens 1253 (EA).

Hoslundia opposita Vahl. Habit: Shrub. Habitat: Bushland wooded grassland, 1-2000 m. Vouchers: FOKP 11441 (EA, HIB), Mbuni 177 (EA), Webster 8969 AE. 
Leonotis mollissima Gürke. Habit: Herb. Habitat: Disturbed grassland, 15002600 m. Voucher: SAJIT 004845 (EA, HIB).

Leonotis nepetifolia (L.) R. Br. Habit: Shrub. Habitat: Disturbed grassland, 9002300 m. Voucher: SAJIT 004845 (EA, HIB).

Leonotis ocymifolia var. raineriana (Vis.) Iwarsson. Habit: Shrub. Habitat: Montane forest zone, 1770-3360 m. Vouchers: FOKP 942 \& 11311 (EA, HIB), Part II Botany 53 (EA).

Leucas argentea Gürke. Habit: Shrub. Habitat: Upland grassland, 1200-2710 m. Vouchers: Thulin \& Tidigs 123 (EA), Dale 3408 (EA).

Leucas calostachys var. fasciculata (Baker) Sebald. Habit: Herb. Habitat: Grassland, 1600-2800 m. Vouchers: FOKP 1217, 11348 \& 11371 (EA, HIB), Mbuni 10 \& 754 (EA).

Leucas deflexa Hook. f. Habit: Herb. Habitat: Upland grassland, 1600-2600 m. Vouchers: SAJIT 006803 (EA, HIB), FOKP 10903 \& 11312 (EA, HIB), Mbuni 048 (EA).

Leucas masaiensis Oliv. Habit: Herb. Habitat: Upland grassland, 1280-2735 m. Voucher: Thulin \& Tidigs 272 (EA).

Leucas martinicensis (Jacq.) R. Br. Habit: Herb. Habitat: Disturbed soil especially on farmland, 0-2200 m. Vouchers: FOKP 11696 (EA), Mbuni 696 (EA).

Leucas oligocephala Hook. f. Habit: Shrub. Habitat: Wooded grassland, 16002990 m. Voucher: Symes 753 (EA).

Lippia javanica (Burm. f.) Spreng. Habit: Shrub. Habitat: Wooded bushland, 13002100 m. Vouchers: FOKP 11448 (EA, HIB), Mbuni 184 (EA).

Mentha aquatica L. Habit: Herb. Habitat: Upland forest, 1950-2600 m. Voucher: Symes 213 (EA).

Micromeria imbricata (Forssk.) C. Chr. Habit: Herb. Habitat: Upland dry grassland, 1580-3600 m. Vouchers: FOKP 11546 \& 14499 (EA, HIB), Mbuni 235 \& 283 (EA), Thulin \& Tidigs 74 (EA).

Nepeta azurea R. Br. ex Benth. Habit: Herb. Habitat: Bushland, 1800-3600 m. Voucher: Mabberley \& McCall 248 (EA).

Ocimum americanum L. Habit: Herb. Habitat: Bushland, Disturbed grassland, 0-1850 m. Vouchers: FOKP 11472 (EA, HIB), Mbuni 208 (EA).

Ocimum decumbens Gürke. Habit: Shrub. Habitat: Bushland, 1950-3000 m. Voucher: Maas 4754b (EA).

Ocimum gratissimum L. Habit: Shrub. Habitat: Disturbed grassland, 1100-2325 m. Voucher: Symes 156 (EA).

Ocimum kilimandscharicum Gürke Habit: Shrub. Habitat: Stony, Riverine grassland, 1100-2350 m. Voucher: SAJIT 004746 (EA, HIB).

Ocimum lamiifolium Hochst. ex Benth. Habit: Shrub. Habitat: Montane forest, 1550-2340 m. Vouchers: FOKP 11350, 11355 \& 11633 (EA, HIB), Mbuni 086 \& 633 (EA, HIB), Webster 8975 (EA).

Orthosiphon rubicundus (D. Don) Benth. Habit: Herb. Habitat: Wooded grassland, 1600-2635 m. Voucher: Symes 73 (EA, HIB). 
Orthosiphon schimperi Benth. Habit: Herb. Habitat: Wooded grassland, 16002635 m. Voucher: Napier 1935 (EA).

Platostoma rotundifolium (Briq.) A.J. Paton. Habit: Herb. Habitat: Swampy grassland, 1500-2800 m. Vouchers: SAJIT Z0021 (EA, HIB), FOKP 1222 (EA, HIB).

Plectranthus agnewii Lukhoba \& A.J. Paton. Habit: Herb. Habitat: Rocky grassland, 1700-1900 m. Voucher: Thomas 3396 (EA).

Plectranthus amboinicus (Lour.) Spreng. Habit: Shrub. Habitat: Rocky, bushland, 130-1870 m. Voucher: Tweedie 4264 (EA).

Plectranthus autranii (Briq.) Erhardt, Götz \& Seybold Habit: Shrub. Habitat: Wet forest, streamside, 1500-3000 m. Voucher: Thulin \& Tidigs 176 (EA).

Plectranthus barbatus Andrews. Habit: Shrub. Habitat: Bushland, rocky grassland, 880-2950 m. Vouchers: FOKP 967, 1037, 1883, 11360, 11368, \& 11425 (EA, HIB), Mbuni 160 (EA), Tweedie 4263 (EA), Symes 655 (EA).

Plectranthus bojeri (Benth.) Hedge Habit: Herb. Habitat: Grassland, 1200-2670 m. Voucher: Pole et al. 1514 (EA).

Plectranthus caninus Roth. Habit: Herb. Habitat: Disturbed dry rocky area, 11002500 m. Vouchers: SAJIT 004820 (EA, HIB), FOKP 11343 (EA, HIB).

Plectranthus defoliatus Hochst. ex Benth. Habit: Herb. Habitat: Open grassland, 2000-2880 m. Voucher: Tweedie 3728 (EA).

Plectranthus dupuisii (Briq.) A.J. Paton Habit: Shrub. Habitat: Grazed grassland, 2032-3000 m. Vouchers: SAJIT Z008, Symes 752.

Plectranthus edulis Agnew. Habit: Shrub. Habitat: Grassland, 2032-3000 m. Voucher: FOKP 994 (EA, HIB).

Plectranthus grandicalyx (E.A. Bruce) J.K. Morton. Habit: Shrub. Habitat: Grassland, 2000-3000 m. Voucher: Rauh 652 (EA).

Plectranthus kamerunensis Gürke Habit: Shrub. Habitat: Montane forest zone, 1500-2950 m. Vouchers: FOKP 11365 (EA, HIB), Mbuni 413 (EA).

Plectranthus lactiflorus (Vatke) Angew Habit: Shrub. Habitat: Wooded grassland, 1100-2500 m. Voucher: Webster 8980 (EA).

Plectranthus longipes Baker Habit: Herb. Habitat: Woodland, 700-2440 m. Voucher: SAJIT Z0046 (EA, HIB).

Plectranthus ornatus Codd. Habit: Herb. Habitat: Acacia, Commiphora bushland, 1000-2790 m. Voucher: Gillett 18495 (EA).

Plectranthus punctatus (L. f.) L'Hér. Habit: Herb. Habitat: Disturbed montane, bamboo forest, 1950-3350 m. Vouchers: FOKP 1132 (EA, HIB), Mbuni 090 (EA), Thulin \& Tidigs 51 (EA).

Plectranthus tetradenifolius A.J. Paton Habit: Herb. Habitat: Rocky places, 9502325 m. Voucher: Mathew 6170 (EA).

Plectranthus xylopodus Lukhoba \& A.J. Paton Habit: Herb. Habitat: Dry grassland, bushland, 100-1400 m. Vouchers: SAJIT 004837 (EA, HIB), Cymes 293 (EA).

Pycnostachys eminii Gürke Habit: Shrub. Habitat: Riverine and forest margin, 1600-1800 m. Vouchers: FOKP 11734 (EA), Mbuni 734 (EA). 
Pycnostachys meyeri Gürke ex Engl. Habit: Herb. Habitat: Bamboo zone, secondary bushland, 1900-3000 m. Vouchers: FOKP 11274, 11301 \& 11346 (EA, HIB), Mbuni 010 (EA).

Pycnostachys stuhlmannii Gürke. Habit: Herb. Habitat: Marshes, 1860-2400 m. Voucher: Symes 202 (EA).

Rotheca myricoides (Hochst.) Steane \& Mabb. Habit: Shrub. Habitat: Bushland, 1180-2120 m. Vouchers: FOKP 11566 \& 11691 (EA), Mbuni 303, 691 (EA).

Salvia merjamie Forssk. Habit: Herb. Habitat: Grassland, 1800-3500 m. Voucher: Lucas 167 (EA).

Salvia nilotica Juss. ex Jacq. Habit: Herb. Habitat: Grassland, 1800-3500 m. Vouchers: FOKP $11534 \& 11704$ (EA, HIB), Mbuni $270 \& 704$ (EA), Symes 75 (EA).

Scutellaria paucifolia Baker Habit: Herb. Habitat: Short grassland, 1550-2200 m. Voucher: Webster 8978 (EA).

Scutellaria schweinfurthii Briq. Habit: Herb. Habitat: Short grassland, 15501950 m. Voucher: Symes 33 (EA).

Scutellaria violascens Gürke Habit: Herb. Habitat: wooded grassland, 1920-2480 m. Vouchers: SAJIT 005107 (EA, HIB), Chater 1932 (EA).

Satureja abyssinica (Benth.) Briq. Habit: Herb. Habitat: Wooded grassland, 19202500 m. Vouchers: FOKP 1085, Bridson 101 (EA).

Micromeria biflora (Buch.-Ham. ex D. Don) Benth. Habit: Habitat: Grassland, 1580-3300 m. Voucher: FOKP 1223 (EA, HIB).

Satureja imbricata (Forssk.) Briq. Habit: Shrub. Habitat: Dry grassland, 15803500 m. Voucher: Part II Botany 79 (EA).

Satureja pseudosimensis Brenan. Habit: Shrub. Habitat: Grassland, 1400-2900 m. Voucher: Symes 622 (EA).

Stachys alpigena T.C.E. Fr. Habit: Herb. Habitat: Alpine zone, 2940-3550 m. Vouchers: FOKP 11325 (EA, HIB), Mbuni 061 (EA).

Tetradenia riparia (Hochst.) Codd. Habit: Shrub. Habitat: Rocky slopes, 6002200 m. Voucher: SAJIT 004839 (EA, HIB).

Tinnea aethiopica Kotschy ex Hook. f. Habit: Shrub. Habitat: Forest thicket, forest margin, Upland woodland up to $2200 \mathrm{~m}$. Vouchers: FOKP 11692, Mbuni 692 (EA), Lucas 160 (EA).

Rotheca myricoides (Hochst.) Steane \& Mabb. Habit: Shrub. Habitat: Grazed bushland, 1180-2120 m. Vouchers: FOKP 11566 (EA, HIB), Mbuni 303 (EA).

\section{F119. Orobanchaceae}

9 Genera, 14 Species

Alectra parasitica A. Rich. Habit: Parasitic herb. Habitat: Dry areas, 434-1670 m. Voucher: Mabberley \& McCall 276 (EA).

Alectra sessiliflora (Vahl) Kuntze. Habit: Parasitic herb. Habitat: Dry areas, 5002000 m. Voucher: Bogdan 5302 (EA).

Bartsia longiflora Hochst. ex Benth. Habit: Shrub. Habitat: Heath vegetation, 2450-3480 m. Voucher: Mabberley 299 (EA). 
Bartsia trixago L. Habit: Herb. Habitat: Heath vegetation, 900-3100 m. Vouchers: Symes 645 (EA), Tweedie 4201 (EA), Mabberley 589 (EA).

Buchnera nuttii Skan. Habit: Parasitic herb. Habitat: Wooded grassland, 13002170 m. Vouchers: Gardener 3723 (EA), Webster 8979 (EA).

Buchnera scabridula E.A. Bruce Habit: Herb. Habitat: Grassland, 2640-2940 m. Vouchers: Mabberley \& McCall 303 (EA), Irwin 370 (EA).

Buttonia natalensis McKen ex Benth Habit: Climber. Habitat: Dry bushland, 1800 -1860 m. Voucher: SAJIT 005142 (EA, HIB).

Cycnium tenuisectum (Standl.) O.J. Hansen Habit: Herb. Habitat: Upland grassy marshes, 1800-3200 m. Vouchers: SAJIT 005103 (EA, HIB), Thulin \&Tidigs 129 (EA), Lucas 213 (EA).

Hedbergia abyssinica (Benth.) Molau Habit: Herb. Habitat: Upland grassland around, $2700 \mathrm{~m}$. Voucher: Thulin \& Tidigs 157 (EA).

Orobanche ramosa L. Habit: Herb. Habitat: Upland grassland, 1735-2250 m. Voucher: SAJIT 004782 (EA, HIB).

Orobanche minor Sm. Habit: Herb. Habitat: Upland grassland, 800-2480 m. Vouchers: SAJIT 006900 (EA, HIB), Thulin \& Tidigs 177 (EA).

Sopubia karaguensis var. welwitschii (Engl.) O.J. Hansen Habit: Herb. Habitat: Montane grassland, 500-3400 m. Voucher: Tweedie 2980 (EA).

Sopubia ramosa (Hochst.) Hochst. Habit: Herb. Habitat: Wooded grassland, 1600 2170m. Vouchers: Graham 324 (EA), Agnew et al. 10581 (EA), Gardner 3724 (EA).

Striga asiatica (L.) Kuntze Habit: Parasitic herb. Habitat: Upland grassland, 0-2480 m. Voucher: Mabberley 86 (EA).

\section{Lentibulariaceae}

2 Genera, 4 Species

Genlisea hispidula Stapf Habit: Herb. Habitat: Grassland pools, 1860-2400 m. Voucher: Thulin \& Tidings 182 (EA).

Utricularia arenaria A. DC. Habit: Herb. Habitat: Seasonal swamps, 380-2950 m. Voucher: FOKP 1140 (EA, HIB).

Utricularia livida E. Mey. Habit: Herb. Habitat: Seasonal pools, 2325-2730 m. Vouchers: SAJIT 006914, FOKP 1140 \& 11014 (EA, HIB).

Utricularia prehensilis E. Mey. Habit: Herb. Habitat: Upland marshes, 18602400 m. Voucher: Thulin \& Tidings 181 (EA).

\section{F121. Acanthaceae}

17 Genera, 35 Species

Acanthus eminens C.B. Clarke Habit: Shrub. Habitat: Dry forest, amongst bamboo, 1500-2800 m. Vouchers: FOKP 1002 (EA, HIB), Part II Botany Class 11 (EA). Asystasia mysorensis (Roth) T. Anderson Habit: Herb. Habitat: Bushland, 11602100 m. Voucher: SAJIT 005148 (EA, HIB). 
Barleria grandicalyx Lindau Habit: Herb. Habitat: Savannah, Wooded grassland, 1100-2500 m. Vouchers: Napier 1949 (EA), Symes 35 (EA).

Blepharis edulis (Forssk.) Pers. Habit: Herb. Habitat: Grassland, 140-2015 m. Voucher: SAJIT Z0066 (HIB).

Blepharis maderaspatensis (L.) B. Heyne ex Roth Habit: Herb. Habitat: Grassland, bushland, 1000-3300 m. Voucher: SAJIT Z0044 (HIB).

Crossandra massaica Mildbr. Habit: Herb. Habitat: Bushland, 1200-2700 m. Vouchers: FOKP 11455 \& 11663 (EA), Mbuni 191 \& 663 (EA).

Crossandra nilotica Oliv. Habit: Herb. Habitat: Bushland, 1200-1500 m. Voucher: Mabberley 793 (EA).

Dyschoriste thunbergiiflora (S. Moore) Lindau Habit: Shrub. Habitat: Acacia, Commiphora bush, 600-1450 m. Vouchers: SAJIT Z0040 (EA, HIB), Mabberley \& McCall 265 (EA).

Dicliptera laxata C.B. Clarke Habit: Herb. Habitat: Forest undergrowth, 13002800 m. Voucher: Part II Botany 31 (EA).

Dicliptera latibracteata I. Darbysh. Habit: Herb. Habitat: Forest undergrowth, grassland, 2200-2900 m. Voucher: Hepper 5033 (EA).

Dicliptera maculata Nees Habit: Climber. Habitat: Grassland, 1700-2800 m. Voucher: FOKP 11398 (EA, HIB).

Hypoestes triflora (Forssk.) Roem. \& Schult. Habit: Herb. Habitat: Grassland, bushland, 1600-3300 m. Vouchers: FOKP 1153 \& 11305 (EA, HIB), Mbuni 041 (EA), Lind 2828 (EA).

Hypoestes forskaolii subsp. hildebrandtii (Lindau) I. Darbysh. Habit: Herb. Habitat: Dry grassland, bushland forest, 0-2820 m. Vouchers: FOKP 932, 11296 \& 11626, (EA, HIB), Mbuni $032 \& 626$ (EA, HIB).

Hygrophila auriculata (Schumach.) Heine Habit: Herb. Habitat: Grassland, about 2000 m. Voucher: Ivens 1285 (EA).

Hygrophila schulli M.R. Almeida \& S.M. Almeida Habit: Herb. Habitat: Bushland, up to $2170 \mathrm{~m}$. Voucher: Ivens 1285 (EA).

Isoglossa gregoryi (S. Moore) Lindau Habit: Herb. Habitat: Montane and bamboo forest, 1900-2820 m. Voucher: FOKP 1745 (EA, HIB).

Isoglossa substrobilina C.B. Clarke Habit: Herb. Habitat: Montane rain forest, 1600-2200 m. Vouchers: FOKP $9481120 \& 1747$ (EA, HIB).

Justicia anagalloides (Nees) T. Anderson Habit: Herb. Habitat: Grassland, 10202500 m. Vouchers: Geesteranus 4785 (EA), Symes 39 (EA).

Justicia betonica L. Habit: Herb. Habitat: Grassland, Wet forests, riversides, 7002220 m. Voucher: Tweedie 2958 (EA).

Justicia calyculata Deflers Habit: Herb. Habitat: Bushland, 1000-2200 m. Voucher: SAJIT Z0043 (HIB).

Justicia exigua S. Moore. Habit: Herb. Habitat: Grassland, 1000-2200 m. Voucher: Symes 272 (EA).

Justicia diclipteroides Lindau Habit: Herb. Habitat: Woodland 1600-2325 m. Vouchers: FOKP 1088 \& 11555 (EA, HIB), Mbuni 292 \& 744 (EA), Geesteranus 4793 (EA). 
Justicia flava (Forssk.) Vahl Habit: Herb. Habitat: Wooded grassland, 1400-2300 m., Vouchers: SAJIT 004725 (EA, HIB), Geesteranus 6375 (EA).

Justicia leikpiensis S. Moore Habit: Herb. Habitat: Grassland, 2130-2480 m. Vouchers: Napier 1914 (EA), Mainwaring 18 (EA).

Justicia pinguior C.B. Clarke Habit: Herb. Habitat: Wooded grassland, 17202170 m. Voucher: Symes 40 (EA).

Justicia unyorensis S. Moore. Habit: Herb. Habitat: Montane rain forest, 2170$3100 \mathrm{~m}$. Voucher: Trelawny 4389 (EA).

Justicia uncinulata Oliv. ex C.B. Clarke Habit: Herb. Habitat: Wooded grassland, 1700-2200 m. Voucher: Symes 39 (EA).

Lepidagathis collina (Endl.) Milne-Redh. Habit: Herb. Habitat: Bushland, 18002170 m. Voucher: Heliz et al. 853 (EA).

Megalochlamys kenyensis Vollesen Habit: Shrub. Habitat: Wooded grassland, bushland, 1050-1450 m. Voucher: Tweedie 4265 (EA).

Megalochlamys violacea (Vahl) Vollesen. Habit: Shrub. Habitat: Acacia bushland, 150-1420 m. Voucher: Tweedie 4265 (EA).

Mimulopsis solmsii Schweinf. Habit: Herb. Habitat: Bushland, 1700-2300 m. Vouchers: SAJIT Z0009 (EA, HIB), FOKP 1073 \& 1202 (EA, HIB), Tweedie 3229 (EA).

Schaueria paniculata Nees Habit: Herb. Habitat: Bushland, 1800-2170 m. Vouchers: FOKP 11484 (EA), Mbuni 220 (EA).

Thunbergia alata Bojer ex Sims Habit: Herb. Habitat: Grassland, bushland, 1003000 m. Voucher: FOKP 11275 \& 11568 (EA, HIB), Mbuni 305 \& 662 (EA), Tweedie 2703 (EA).

Thunbergia paulitschkeana Beck Habit: Herb. Habitat: Grassland, 1200-3000 m. Voucher: Hepper 5024 (EA).

\section{F122. Bignoniaceae}

1 Genus, 1 Species

Stereospermum kunthianum Cham. Habit: Tree. Habitat: Rocky bushland, wooded grassland, 900-2100m. Voucher: Bogdan 265 (EA).

\section{F123. Verbenaceae}

4 Genera, 10 Species

Hebenstreitia rariflora A. Terracc. Habit: Herb. Habitat: Heathland to $3550 \mathrm{~m}$. Vouchers: FOKP 11390 (EA, HIB), Mbuni 125 (EA).

Lantana trifolia L. Habit: Shrub. Habitat: Bushland, 900-2350 m. Vouchers: FOKP 1036, 1287, 11322, $11685 \& 11742$ (EA, HIB), Mbuni 058, $685 \& 742$ (EA), Lind 5095 (EA).

Lippia grandifolia Hochst. ex A. Rich. Habit: Shrub. Habitat: Wooded grassland, 750-2250 m. Voucher: Lind 5096 (EA). 
Lippia javanica (Burm. f.) Spreng. Habit: Shrub. Habitat: Wooded grassland, bushland, 1050-2300 m. Vouchers: FOKP $1886 \& 11684$ (EA, HIB), Mbuni 684 (EA).

Lippia kituiensis Vatke Habit: Shrub. Habitat: Bushland, 450-2560 m. Voucher: Tweedie 3736 (EA).

Lippia rehmannii H. Pearson Habit: Shrub. Habitat: Grassland, Bushland, 7502250 m. Voucher: Symes 29 (EA).

Lippia woodii Moldenke Habit: Herb. Habitat: Burnt grassland, 1550-2170 m. Voucher: Napier 1928 (EA).

Verbena aristigera S. Moore Habit: Herb. Habitat: Open forest, 1650-2110 m. Voucher: SAJIT 004738 (EA, HIB).

Verbena bonariensis L. Habit: Herb. Habitat: Bushland, Disturbed areas, 18002650 m. Vouchers: FOKP 1282 \& 11557 (EA, HIB), Mbuni 294 (EA).

Verbena rigida Spreng. Habit: Herb. Habitat: Roadside verge, 1660-2100 m. Voucher: Dyson 568 (EA).

\section{F124. Aquifoliaceae}

1 Genus, 1 Species

Ilex mitis (L.) Radlk. Habit: Tree. Habitat: Upland riverine forest, 1450-3150 m. Vouchers: SAJIT 006912 (EA, HIB), FOKP 1013, 1227, 11374 \& 11549 (EA, HIB), Mbuni 110, 286 (EA).

\section{F125. Campanulaceae}

4 Genera, 15 Species

Canarina eminii Asch. \& Schweinf. Habit: Epiphyte. Habitat: Wet forest, 2100$2650 \mathrm{~m}$. Voucher: Thulin \& Tidigs 59 (EA).

Lobelia aberdarica R.E. Fr. \& T.C.E. Fr. Habit: Shrub. Habitat: Moorland, Swamps, 1860-3350 m. Vouchers: Thulin \& Tidigs 105 (EA), Townsend 2387 (EA).

Lobelia cheranganiensis Thulin Habit: Herb. Habitat: Upland Forest \& Moorland, 2750-3300 m. Vouchers: SAJIT 006863 (EA, HIB), FOKP 10963 (EA, HIB), Tweedie 3883 (EA), Knox 3379 (EA), Thulin \& Tidigs 220 (EA).

Lobelia deckenii (Asch.) Hemsl. Habit: Shrub. Habitat: Moorland up to $3550 \mathrm{~m}$. Voucher: Mabberley \& McCall 186 (EA).

Lobelia duriprati T.C.E. Fr. Habit: Herb. Habitat: Wet montane, 1700-3550 m. Voucher: Thulin \& Tidigs 220 (EA).

Lobelia giberroa Hemsl. Habit: Shrub. Habitat: Forest margin, 1200-3000 m. Vouchers: FOKP 962, $1232 \& 11539$ (EA, HIB), Mbuni 275 (EA), Verdcourt 2437 (EA), Knox $3790 \& 3852$ (EA).

Lobelia holstii Engl. Habit: Herb. Habitat: Grassland 1800-3520 m. Vouchers: SAJIT 004798, Newbould 2440 (EA).

Lobelia minutula Engl. Habit: Herb. Habitat: Disturbed alpine, 1700-3500 m. Voucher: Thulin \& Tidigs 198 (EA). 
Monopsis stellarioides (C. Presl) Urb. Habit: Herb. Habitat: Grassland, 16503500 m. Vouchers: FOKP 11532 \& 11540 (EA, HIB), Mbuni 276 (EA), Mabberley \& McCall 97 (EA).

Monopsis zeyheri (Sond.) Thulin Habit: Herb. Habitat: Wet grassland about $1600 \mathrm{~m}$. Voucher: SAJIT 005137 (EA, HIB).

Wablenbergia capillacea (L. f.) A. DC. Habit: Herb. Habitat: Bamboo zone, Rocky soils, 2300m-2950 m. Vouchers: Bogdan 4987 (EA), Symes 620 (EA).

Wablenbergia krebsii Cham. Habit: Herb. Habitat: Grassland. Lower alpine, Heath zones, 2130-3600 m. Voucher: Napier 1992 (EA).

Wablenbergia krebsii subsp. arguta (Hook. f.) Thulin. Habit: Herb. Habitat: Grassland, in lower alpine and heath zone, 2100-3550 m. Voucher: Thulin \& Tidigs 70 (EA).

Wablenbergia scottii Thulin Habit: Herb. Habitat: Wooded grassland, 2300-2700 m. Vouchers: Thulin \& Tidigs 131 (EA), Verdcourt 2427 (EA).

Wablenbergia silenoides Hochst. ex A. Rich. Habit: Herb. Habitat: Montane grassland, 1500-2100 m. Voucher: Tweedie 3020 (EA).

\section{F126. Asteraceae}

66 Genera, 165 Species

Acanthospermum glabratum (DC.) Wild Habit: Herb. Habitat: Grassland, 13002500 m. Voucher: SAJIT Z0007 (EA).

Acmella caulirhiza Delile Habit: Herb. Herb. Habitat: Grassland, 600-2600 m. Vouchers: FOKP 1230 \& 11427 (EA) Mbuni 163 (EA).

Ageratina adenophora (Spreng.) R.M. King \& H. Rob. Habit: Shrub. Habitat: Swampy sites, riverine, 1960-2300 m. Vouchers: SAJIT 005063 \& 006809 (EA, HIB), FORK $1806 \& 10909$ (EA, HIB).

Ageratum conyzoides (L.) L. Habit: Herb. Habitat: Disturbed ground, 30-2500 m. Voucher: FOKP 11495 (EA), Mbuni 231 (EA).

Anthemis tigrensis J. Gay ex A. Rich. Habit: Herb. Habitat: Moorland zone, 25003500 m. Vouchers: Mabberley 214, Thulin 203 (EA).

Artemisia afra Jacq. ex Willd. Habit: Shrub. Habitat: Dry shrubby montane grassland, 2000-3550 m. Vouchers: SAJIT 005114 (EA, HIB), Mbuni 725 (EA, HIB), Mabberley \& McCall 241 (EA), Thulin 252 (EA).

Aspilia kotschyi (Sch. Bip. ex Hochst.) Oliv. Habit: Herb. Habitat: Grassland, waste land, 10-1900 m. Voucher: Carter \& Stanard 52 (EA).

Aspilia pluriseta Schweinf. ex Schweinf. Habit: Herb. Habitat: Bushed grassland, 1050-2400 m. Vouchers: FOKP 11492 (EA), Mbuni 228 (EA), Symes 98 (EA).

Athrixia rosmarinifolia (Sch. Bip. ex Walp.) Oliv. \& Hiern. Habit: Herb. Habitat: Highland shrubland, 2600-3400 m. Vouchers: SAJIT 005119 (EA, HIB), Tweedie 3904 (EA).

Baccharoides dumicola (S. Moore) "Isawumi, El-Ghazaly \& B. Nord." Habit: Herb. Habitat: Woodland, grassland edge, 1200-2300 m. Voucher: Webster 8898 (EA). 
Baccharoides adoensis (Sch. Bip. ex Walp.) H. Rob. Habit: Shrub. Habitat: Bushland, 125-2100 m. Voucher: FOKP 11386 (EA, HIB).

Berkheya spekeana Oliv. Habit: Herb. Habitat: Wooded grassland, 1800-3100 m. Vouchers: SAJIT 005124 (EA, HIB), FOKP 1905 (EA, HIB), Symes 157 (EA).

Bidens buchneri (Klatt) Sherff Habit: Herb. Habitat: Wooded grassland, 14501500 m. Vouchers: Napier 1924 (EA), Agnew et al. 312 (EA).

Bidens elgonensis (Sherff) Agnew Habit: Herb. Habitat: Erica bushland, heath zone, 1900-3550 m. Vouchers: FOKP 11280 (EA, HIB), Mbuni 016 (EA), Tweedie 338 (EA).

Bidens flagellata (Sherff) Mesfin Habit: Herb. Habitat: Grassland, 1600-3300 m. Voucher: Thulin \& Tidigs 264 (EA).

Bidens kilimandscharica (O. Hoffm.) Sherff Habit: Shrub. Habitat: Upland forest edges, 1500-2800 m. Vouchers: FOKP 11569 (EA, HIB), Mbuni 306 (EA), Tweedie 4214 (EA).

Bidens lineariloba Oliv. Habit: Herb. Habitat: Bushland, wooded land, 500-1800 m. Voucher: Tweedie 369 (EA).

Bidens pilosa L. Habit: Herb. Habitat: Highland areas, 400-2500 m. Vouchers: FOKP 11434 (EA), Mbuni 170 (EA).

Blumea axillaris (Lam.) DC. Habit: Herb. Habitat: Disturbed areas, 1500-2700 m. Voucher: FOKP 11366 (EA), Mbuni 102 (EA).

Bothriocline fusca (S. Moore) M.G. Gilbert Habit: Shrub. Habitat: Upper forest zones, 2000-3550 m. Voucher: FOKP 11377 (EA, HIB).

Bothriocline longipes (Oliv. \& Hiern) N.E. Br. Habit: Shrub. Habitat: Forest margin, 1500-2800 m. Vouchers: FOKP 11519 (EA, HIB), Mbuni 255 (EA).

Bothriocline monticola (M. Taylor) Wech. Habit: Shrub. Habitat: Forest margin, about 2800 m. Voucher: Tweedie 4200 (EA).

Bothriocline trifoliata (De Wild. \& Muschl.) Wild \& G.V. Pope Habit: Shrub. Habitat: Montane rain forest, 1950-2500 m. Voucher: Thulin \& Tidigs 209 (EA).

Carduus chamaecephalus (Vatke) Oliv. \& Hiern Habit: Herb. Habitat: Grassland, alpine, subalpine, 2400-3550 m. Voucher: Gillett 18422 (EA).

Carduus keniensis R.E. Fr. Habit: Herb. Habitat: Grassland, 2950-3550 m. Vouchers: Mbuni 098 (EA), Mabberley \& McCall 212 (EA).

Carduus nyassanus (S. Moore) R.E. Fr. Habit: Herb. Habitat: Montane forest, 2700-3550 m. SAJIT 004773 (EA, HIB), Vouchers: FOKP 11363 (EA, HIB), Mbuni 099 (EA), Symes 402 (EA).

Carduus schimperi Sch. Bip. Habit: Herb. Habitat: Grassland \& Moorland, 25503550 m. Voucher: Gillett 18422 (EA).

Centaurea praecox Oliv. \& Hierm Habit: Herb. Habitat: Wooded grassland, 1600 2300 m. Vouchers: Symes 523 (EA), Tweedie 1519 (EA), Thairu 143 (EA), Webster 8856 (EA).

Cineraria grandiflora Vatke Habit: Herb. Habitat: Wooded grassland, 1800-3500 m. Voucher: Thulin 72 (EA). 
Cineraria deltoidea Sond. Habit: Herb. Habitat: Roadsides, forest edges cliffs, 18903550 m. Vouchers: FOKP 1131 \& 11339 (EA, HIB), Mbuni 075 (EA), Thulin $\&$ Mhoro 72 (EA).

Cirsium vulgare (Savi) Ten. Habit: Herb. Habitat: Arable land, 1790-2400 m. Vouchers: FOKP 1369 \& 11362 (EA, HIB), Mbuni 098 \& 105 (EA).

Conyza aegyptiaca (L.) Dryand. ex Aiton Habit: Herb. Habitat: Disturbed places, 300-2000 m. Voucher: Symes 64 (EA).

Conyza agnewii Mesfin Habit: Herb. Habitat: Wooded grassland around $1850 \mathrm{~m}$. Voucher: Napier 1931 (EA).

Conyza newii Oliv. \& Hiern. Habit: Shrub. Habitat: Montane forest, 1600-2500 m. Vouchers: FOKP 11321 (EA, HIB) Mbuni, 057 (EA).

Conyza pyrrhopappa Sch. Bip. ex A. Rich. Habit: Shrub. Habitat: Upland bushland, 1500-2200 m. Vouchers: SAJIT 006854 (EA, HIB), FOKP 11442 (EA, HIB), Mbuni 178 (EA).

Conyza steudelii Sch. Bip. ex A. Rich. Habit: Herb. Habitat: Medium altitude, 1500-3500 m. Vouchers: FOKP 11346 (EA, HIB), Mbuni 082 (EA).

Conyza subscaposa O. Hoffm. Habit: Herb. Habitat: Alpine and subalpine grassland, 3000-3500 m. Voucher: Symes 618 (EA).

Conyza vernonioides (Sch. Bip. ex A. Rich.) Wild Habit: Shrub. Habitat: Moorland and bamboo, 3000-3550 m. Voucher: Tweedie 3901 (EA).

Cotula abyssinica Sch. Bip. ex A. Rich. Habit: Herb. Habitat: Alpine, upland grassland, 2700-3500 m. Vouchers: Bogdan 496 (EA), Symes 643 (EA), and Mabberley 216 (EA).

Crassocephalum bojeri (DC.) Robyns Habit: Herb. Habitat: Montane forest, 15003200 m. Voucher: Thulin 159 (EA).

Crassocephalum montuosum (S. Moore) Milne-Redh. Habit: Herb. Habitat: Montane forest, 1500-3260 m. Vouchers: FOKP 1724 (EA, HIB), Chetham 1 (EA), Thulin \& Tidigs 266 (EA).

Crassocephalum $\times$ picridifolium (DC.) S. Moore Habit: Herb. Habitat: Swamps and river edges, 1300-2500 m. Vouchers: SAJIT 004806 (EA, HIB), FOKP 11503 (EA, HIB), Mbuni 239 (EA), Symes 269 (EA).

Crassocephalum rubens var. sarcobasis (Bojer ex DC.) C. Jeffrey \& Beentje Habit: Herb. Habitat: Disturbed, cultivated, higher altitude, 1500-2800 m. Vouchers: Verdcourt 746 (EA), Thulin 188 (EA), Symes 205 (EA).

Crassocephalum vitellinum (Benth.) S. Moore Habit: Herb. Habitat: Upland forest and woodland, 1500-2800 m. Vouchers: FOKP 981 (EA, HIB), Thulin 158 (EA).

Crepis rueppellii Sch. Bip. Habit: Herb. Habitat: Highland grassland, 1600-3200 m. Vouchers: FOKP 1221 (EA, HIB), Townsend 2368 (EA).

Dendrosenecio cheranganiensis (Cotton \& Blakelock) E.B. Knox Habit: Shrub. Habitat: Upper Afromontane evergreen, 3000-3550 m. Vouchers: Dale 3392 (EA), Mabberley 30 (EA). 
Dendrosenecio cheranganiensis subsp. cheranganiensis (Cottton \& Blakeclock)

E.B. Knox Habit: Shrub. Habitat: Upper Afromontane evergreen, 3000-3550 m. Vouchers: Merbbery 512 (EA), Knox 719 (EA), Beentje 3058 (EA).

Dendrosenecio cheranganiensis subsp. dalei (Cotton \& Blakelock) E.B. Knox Habit: Shrub. Habitat: Upper Afromontane zone, 3000-3550 m. Voucher: Mabberley \& McCall 209 (EA).

Dichrocephala integrifolia (L. f.) Kuntze Habit: Herb. Habitat: Disturbed wet habitat, 1700-3550 m. Vouchers: FOKP 111406 (EA, HIB), Mbuni 142 (EA, HIB).

Dicoma tomentosa Cass. Habit: Herb. Habitat: Dry bushland, 480-1500 m. Voucher: Carter \& Stanard 13 (EA).

Emilia abyssinica (Sch. Bip. ex A. Rich.) C. Jeffrey Habit: Herb. Habitat: Cultivated land, 800-1900 m. Voucher: Tweedie 1922 (EA).

Emilia coccinea (Sims) G. Don Habit: Herb. Habitat: Roadside, wasteland, 0-2000 m. Voucher: Carter \& Stannard 15 (EA).

Emilia debilis S. Moore Habit: Herb. Habitat: Disturbed marshes, 1600-2200 m. Voucher: Symes 751 (EA)

Echinops aberdaricus R.E. Fr. Habit: Herb. Habitat: Subalpine grassland, 29003300 m. Voucher: Thulin 143 (EA).

Echinops angustilobus S. Moore Habit: Herb. Habitat: Higher altitudes, grassland, 2000-2800 m. Vouchers: FOKP 1139 (EA, HIB), Bogdan 5008 (EA), Townsend 2376 (EA).

Echinops amplexicaulis Oliv. Habit: Herb. Habitat: Wooded grassland, 1300$2700 \mathrm{~m}$. Voucher: Thairu 1, Napper 1501 (EA).

Echinops hispidus Fresen. Habit: Herb. Habitat: Upland grassland, 1500-2200 m. Voucher: Symes 403 (EA).

Echinops hoehnelii Schweinf. Habit: Herb. Habitat: High altitude woodland \& heartland, 2200-3500 m. Vouchers: FOKP 11381 (EA), Mbuni $116 \& 118$ (EA).

Echinops lanatus C. Jeffrey \& Mesfin Habit: Herb. Habitat: Montane grassland, 2400-3600 m. Voucher: Tweedie 4183 (EA).

Erigeron bonariensis L. Habit: Herb. Habitat: Disturbed soil, 400-2600 m. Vouchers: FOKP 11338 \& 11724 (EA, HIB), Mbuni 074 \& 724 (EA), Cronquist 2396 (EA).

Euryops brownei S. Moore Habit: Shrub. Habitat: Upper forest level and lower heath zone, 2300-3550 m. Vouchers: SAJIT 004794, Tweedie 391 (EA), Lucas 165 (EA), Wamukoya 129 (EA).

Euryops chrysanthemoides (DC.) B. Nord. Habit: Shrub. Habitat: Heath zone, 2500 3500 m. Vouchers: FOKP 11578 \& 11624 (EA, HIB), Mbuni 315 \& 624 (EA).

Ethulia vernonioides (Schweinf.) M.G. Gilbert Habit: Shrub. Habitat: Grassland, 1650-2750 m. Vouchers: SAJIT 006815 (EA, HIB), FOKP 989, 1803 \& 11550 (EA, HIB), Mbuni 287 (EA), Webster 888 (EA).

Eupatorium adenophorum Spreng. Habit: Herb. Habitat: Riverine, 1750-2500 m. Voucher: Symes 282 (EA).

Eupatorium africanum Oliv. \& Hieron. Habit: Herb. Habitat: Riverine, 17002500 m. Voucher: Symes 31 (EA). 
Felicia abyssinica Sch. Bip. ex A. Rich. Habit: Herb. Habitat: Grassland, 13002500 m. Voucher: Symes 527 (EA).

Galinsoga quadriradiata Ruiz \& Pav. Habit: Herb. Habitat: Wet highland, 18002500 m. Vouchers: FOKP 11426 (EA, HIB), Mbuni 162 (EA).

Galinsoga parviflora Cav. Habit: Herb. Habitat: Wet highland, 300-2300 m. Voucher: Jeffery 272 (EA).

Gerbera hirsuta (Forssk.) Less. Habit: Herb. Habitat: Highland grassland, 15003000 m. Voucher: Mabberley 208 (EA).

Gerbera viridifolia (DC.) Sch. Bip. Habit: Herb. Habitat: Wooded grassland, 16002500 m. Voucher: Symes 52 (EA).

Gerbera piloselloides (L.) Cass. Habit: Herb. Habitat: Upland and highland grassland, 1500-3400 m. Voucher: SAJIT 005106 (EA, HIB).

Gnaphalium unionis Sch. Bip. ex Oliv. \& Hiern Habit: Herb. Habitat: Grassland, 1600-3200 m. Vouchers: FOKP 1794 (EA, HIB), Townsend 238 (EA).

Gnaphalium declinatum L. f. Habit: Herb. Habitat: Grassland, 1600-3000 m. Voucher: Trelawny 4384 (EA).

Gnaphalium luteoalbum L. Habit: Herb. Habitat: Highland grassland, 300-3050 m. Vouchers: Symes 74 (EA), Thulin \& Tidigs 115 (EA).

Guizotia scabra (Vis.) Chiov. Habit: Herb. Habitat: Upland grassland, 13003200 m. Vouchers: Thulin 46 (EA), Lind 5098 (EA).

Guizotia jacksonii (S. Moore) J. Baagøe Habit: Herb. Habitat: Bamboo, heath zone, 2400-3500 m. Voucher: Thulin \& Tidigs 68 (EA).

Gutenbergia rueppellii Sch. Bip. Habit: Herb. Habitat: Rocky grassland, 9002200 m. Voucher: Bogdan 3853 (EA).

Haplocarpha rueppellii (Sch. Bip.) K. Lewin. Habit: Herb. Habitat: Alpine zone, 2600-3550 m. Voucher: SAJIT 005123 (EA, HIB).

Helichrysum argyranthum O. Hoffm. Habit: Shrub. Habitat: Upper forest edge, streamside, heath zone, 2100-3550 m. Vouchers: FOKP 996, 11264, 11374 \& 11340 (EA, HIB), Mbuni 020 (EA).

Helichrysum brownei S. Moore Habit: Shrub. Habitat: Heath zone, 3000-3550 m. Voucher: SAJIT 005111 (EA, HIB).

Helichrysum cymosum (L.) D. Don Habit: Shrub. Habitat: Wooded land, 16002900 m. Voucher: Symes 624 (EA).

Helichrysum ellipticifolium Moeser Habit: Shrub. Habitat: Alpine grassland, 2650 3550 m. Voucher: Knox 3390 (EA).

Helichrysum foetidum (L.) Cass. Habit: Herb. Habitat: Upland forest, forest margin, 2400-3000 m. Vouchers: FOKP 11520 (EA, HIB), Mbuni $256 \& 723$ (EA), Mabberley 583 (EA), Symes 99 (EA).

Helichrysum formosissimum Sch. Bip. Habit: Shrub. Herb. Habitat: Alpine zone, 2300-3500 m. Voucher: Bogdan 5000 (EA).

Helichrysum formosissimum var. guilelmii (Engl.) Mesfin Habit: Herb. Habitat: Heath zone, moorland, upper bamboo zone, 1800-2500 m. Voucher: Mabberley 571 (EA). 
Helichrysum forskablii (J.F. Gmel.) Hilliard \& B.L. Burtt Habit: Herb. Habitat: Rocky grassland, 1200-3500 m. Vouchers: SAJIT 005104 \& 006805 (EA, HIB), FOKP 10905 \& 11538 (EA, HIB), Mbuni 274 (EA), Knox 3352 (EA), Symes 99 (EA).

Helichrysum gerberifolium Sch. Bip. ex A. Rich. Habit: Herb. Habitat: Wooded grassland, 2600-3500 m. Voucher: Symes 25 (EA).

Helichrysum globosum Sch. Bip. Habit: Herb. Habitat: Upland grassland, 18003300 m. Vouchers: FOKP 11478 (EA, HIB), Mbuni 214 (EA).

Helichrysum gloria-dei Chiov. Habit: Shrub. Habitat: Alpine zone, 3300-3550 m. Voucher: SAJIT 005108 (EA, HIB).

Helichrysum kilimanjari Oliv. Habit: Herb. Habitat: Lower alpine zone, 28003500 m. Vouchers: Mabberley \& McCall 218 (EA), Knox 3863 (EA).

Helichrysum maranguense O. Hoffm. Habit: Shrub. Habitat: Montane rain forest, 2250-3500 m. Vouchers: SAJIT 006922 (EA, HIB), FOKP 11022 (EA, HIB).

Helichrysum meyeri-johannis Engl. Habit: Herb. Habitat: Alpine grassland, 29003550 m. Voucher: Dale 3418 (EA).

Helichrysum nandense S. Moore Habit: Shrub. Habitat: Grassland, 2250-2900 m. Vouchers: Knox 3447 (EA), Blake 3080 (EA).

Helichrysum odoratissimum (L.) Sweet Habit: Shrub. Habitat: Dry areas, 10003500 m. Vouchers: FOKP 11537 (EA, HIB), Mbuni 273 (EA), Thulin \& Tidigs 254 (EA).

Helichrysum panduratum var. panduratum O. Hofffm. Habit: Shrub. Habitat: Grassland, 1600-2900 m. Voucher: Symes 396 (EA).

Helichrysum setosum Harv. Habit: Herb. Habitat: Montane forest, 1900-2750 m. Voucher: FOKP 944 (EA, HIB).

Helichrysum schimperi (Sch. Bip. ex A. Rich.) Moeser Habit: Shrub. Habitat: Dry forest 1600-3400 m. Vouchers: FOKP 11340 (EA, HIB), Mbuni 076 (EA).

Helichrysum stenopterum DC. Habit: Herb. Habitat: Upper forest edge, 26603500 m. Vouchers: FOKP 11285 (EA, HIB), Mbuni 021 (EA), Symes 206 (EA).

Hirpicium diffusum (O. Hoffm.) Roessler Habit: Herb. Habitat: Disturbed grassland, 500-2200 m. Vouchers: FOKP 11690 \& 14476 (EA), Mbuni 212 \& 690 (EA).

Inula glomerata Oliv. \& Hiern Habit: Herb. Habitat: Wooded grassland, 15002900 m. Vouchers: Freidberg \& Kaplan 56 (EA), FOKP 11328 (EA, HIB).

Inula mannii (Hook. f.) Oliv. \& Hiern Habit: Herb. Habitat: Wet montane forest, 1700-2750 m. Voucher: Tweedie 4184 (EA).

Kleinia scottioides C. Jeffrey. Habit: Herb. Habitat: Bushland, wooded grassland, 900-1900 m. Voucher: Meyerhoff 112 (EA).

Lactuca capensis Thunb. Habit: Herb. Habitat: Disturbed ground, montane forest, 1900-3200 m. Voucher: Symes 43 (EA).

Lactuca glandulifera Hook. f. Habit: Herb. Habitat: Montane rain forest, 19003290 m. Vouchers: FOKP 1040 (EA, HIB), FOKP 938 (EA).

Lactuca inermis Forssk. Habit: Herb. Habitat: Medium altitude, 500-3300 m. Vouchers: FOKP 11348 (EA, HIB), Mbuni 084 (EA). 
Laggera brevipes Oliv. \& Hiern Habit: Herb. Habitat: Upland grassland, 10502400 m. Vouchers: SAJIT 006881 (EA, HIB), Mbuni 240A (EA).

Laggera crispata (Vahl) Hepper \& J.R.I. Wood Habit: Shrub. Habitat: Grassland and forest margin, 150-2400 m. Vouchers: SAJIT 006801 (EA, HIB), FOKP 939, $1011,10901 \& 11505$ (EA, HIB), Mbuni 241 (EA).

Laggera elatior R.E. Fr. Habit: Herb. Habitat: Wet Montane forest, 100-3300 m. Vouchers: FOKP 11372 (EA, HIB), Mbuni 065 (EA), Mabberley 483 (EA).

Laggera pterodonta (DC.) Sch. Bip. ex Oliv. Habit: Herb. Habitat: Upland grassland, 1050-2400 m. Voucher: FOKP 1011 (EA, HIB).

Melanthera pungens Oliv. \& Hiern Habit: Herb. Habitat: Wooded grassland, 1650 2300 m. Voucher: Napier 1929 (EA, HIB).

Melanthera scandens (Schumach. \& Thonn.) Roberty Habit: Shrub. Habitat: Upland forest, 1300-2200 m. Vouchers: FOKP 11755 (EA, HIB), Mbuni 755 (EA).

Micractis bojeri DC. Habit: Herb. Habitat: Disturbed streamside, 1700-2300 m. Vouchers: FOKP 11409 (EA, HIB), Mbuni 145 (EA).

Microglossa densiflora Hook. f. Habit: Shrub. Habitat: Bushland, 1700-3200 m. Vouchers: SAJIT 006797 (EA, HIB), FOKP 1219 \& 10897 (EA, HIB), Agnew et al. 10462 (EA).

Mikania chenopodifolia Willd. Habit: Climber. Habitat: Riverine forest, 0-2000 m. Vouchers: SAJIT 006921 (EA, HIB), FOKP 946 \& 11021 (EA, HIB).

Mikaniopsis bambuseti (R.E. Fr.) C. Jeffrey Habit: Climber. Habitat: Dry upland forest, bamboo, 2100-3000 m. Vouchers: SAJIT 006929 (EA, HIB), Townsend 2393 (EA).

Nidorella spartioides (O. Hoffm.) Cronquist Habit: Herb. Habitat: Wooded grassland, 1675-2150 m. Voucher: Napier 1920 (EA).

Notonia abyssinica A. Rich. Habit: Herb. Habitat: Bushland, 1600-2800 m. Vouchers: FOKP 11559 (EA, HIB) \& Mbuni 296 (EA).

Pentas parvifolia Hiern Habit: Shrub. Habitat: Bushland and Wooded grassland, 902500 m. Vouchers: FOKP 11467 \& 11670 (EA, HIB) \& Mbuni 203 \& 670 (EA).

Pseudognaphalium luteoalbum subsp. affine (D. Don) Hilliard \& Burtt Habit: Herb. Habitat: Highland grassland, 300-3050 m. Vouchers: FOKP 11303 (EA, HIB), Mbuni 039 (EA), Thulin \& Tidigs 115 (EA).

Psiadia punctulata (DC.) Vatke Habit: Shrub. Habitat: Grassland, bushland, forest edge, 1000-2500 m. Vouchers: FOKP 11465 (EA, HIB), Mbuni $201 \& 748$ (EA).

Senecio hedbergii C. Jeffrey Habit: Shrub. Habitat: Rocky open grassland, Heath Vegetation, 3300-3550 m. Voucher: Thulin \& Tidigs 217 (EA).

Senecio maranguensis O. Hoffm. Habit: Shrub. Habitat: Upper forest edge, 27002900 m. Voucher: SAJIT 005117 (EA, HIB).

Senecio moorei R.E. Fr. Habit: Shrub. Habitat: Grassland, 1800-3500 m. Voucher: Townsend 2381 (EA), Thulin 167 (EA).

Senecio plantagineoides C. Jeffrey Habit: Herb. Habitat: Grassland, 1700-2000 m. Voucher: Powles 12 (EA). 
Senecio pseudosubsessilis C. Jeffrey Habit: Herb. Habitat: Woodland edge, riverine, 1800-3000 m. Vouchers: Tweedie 3379 (EA), Tweedie 2169 (EA).

Senecio purtschelleri Engl. Habit: Herb. Habitat: Alpine zone, 3200-3550 m. Voucher: Beentje 3378 (EA).

Senecio pseudosubsessilis C. Jeffrey Habit: Shrub. Habitat: Woodland edge, riverine, 1800-3000 m. Voucher: Thulin \& Tidigs 217 (EA).

Senecio schweinfurthii O. Hoffm. Habit: Herb. Habitat: Montane, alpine grassland, 2300-3500 m. Vouchers: Tweedie 3763(EA), Thulin \& Tidigs 164 (EA), Part II Botany 5157(EA), Symes 640 (EA).

Senecio subsessilis Oliv. \& Hiern Habit: Herb. Habitat: Montane rain forest, 24003120 m. Voucher: Thulin 187 (EA).

Senecio syringifolius O. Hoffm. Habit: Climber. Habitat: Montane rain forest, 1500-3300 m. Voucher: Townsend 2392 (EA).

Schkubria pinnata (Lam.) Kuntze ex Thell. Habit: Herb. Habitat: Cultivated land, 1000-2220 m. Vouchers: FOKP 11484 (EA), Mbuni 220 (EA).

Solanecio mannii (Hook. f.) C. Jeffrey Habit: Tree. Habitat: Montane forest, 1400 2700 m. Vouchers: FOKP 1110, 1261, 11279 \& 11457 (EA, HIB), Mbuni 193 (EA).

Sonchus afromontanus R.E. Fr. Habit: Herb. Habitat: Wet highland grassland, 2200-3700 m. Voucher: Thulin \& Tidigs 226 (EA).

Sonchus schweinfurthii Oliv. \& Hiern Habit: Herb. Habitat: Montane forest, 17002800 m. Voucher: Thulin \& Tidigs 69 (EA).

Spilanthes mauritiana (A. Rich. ex Pers.) DC. Habit: Herb. Habitat: Swamp areas, 1500-2000 m. Voucher: Hedberg 79 (EA).

Sphaeranthus suaveolens (Forssk.) DC. Habit: Herb. Habitat: Dry stream bed, 12002500 m. Vouchers: FOKP 1729, 11324 \& 11653 (EA, HIB), Mbuni 060, 653 (EA).

Stoebe kilimandscharica O. Hoffm. Habit: Shrub. Habitat: Heath zone and moorland, 2700-3350 m. Vouchers: FOKP 11317 (EA, HIB), Mbuni 053 (EA), Mwangangi \& Kariuki 372 (EA), Thulin \& Tidigs 250 (EA).

Stomatanthes africanus (Oliv. \& Hiern) R.M. King \& H. Rob. Habit: Herb. Habitat: Wooded grassland, 2000-3000 m. Voucher: Symes 282 (EA).

Tarchonanthus camphoratus L. Habit: Shrub. Habitat: Bushland, 1500-2300 m. Voucher: FOKP 11297 (EA, HIB).

Tolpis capensis (L.) Sch. Bip. Habit: Herb. Habitat: Wet grassland, 2000-3100 m. Vouchers: FOKP 11301 (EA, HIB), Mbuni 037 (EA), Townsend 2368b (EA), Hepper \& Field 5031 (EA).

Tridax procumbens (L.) L. Habit: Herb. Habitat: Roadside, below 2300 m. Voucher: SAJIT Z0038 (EA, HIB).

Vernonia amygdalina Delile Habit: Shrub. Habitat: Disturbed cultivated area, 2002300 m. Voucher: FOKP 10934 (EA, HIB).

Vernonia auriculata Griseb. Habit: Shrub. Habitat: Woodland, bushland, 16002850 m. Voucher: FOKP 959 (EA, HIB).

Vernonia auriculifera Hiern Habit: Shrub. Habitat: Woodland, bushland, 16002650 m. Vouchers: SAJIT 006834 (EA, HIB), FOKP 11401 \& 11718 (EA, HIB), Mbuni 137 \& 718 (EA), Symes 268 (EA). 
Vernonia holstii O. Hoffm. Habit: Shrub. Habitat: Dry forest, 1000-2050 m. Voucher: FOKP 11454 (EA, HIB), Mbuni $190 \& 718$ (EA).

Vernonia galamensis subsp. afromontana (R.E. Fr.) M.G. Gilbert Habit: Shrub. Habitat: Woodland edge, 800-2200 m. Vouchers: SAJIT 006896 (EA, HIB), FOKP $11331 \& 11400$ (EA, HIB), Mbuni 067 \& 136 (EA), Napier 1957 (EA).

Vernonia hymenolepis A. Rich. Habit: Herb. Habitat: Bushed grassland, 16002850 m. Vouchers: FOKP 11287 \& 11402 (EA, HIB), Mbuni 023, 713 (EA), Thulin \& Tidigs 125 (EA).

Vernonia karaguensis Oliv. \& Hiern Habit: Herb. Habitat: Wooded grassland, 1200-2500 m. Voucher: Webster 8894 (EA).

Vernonia lasiopus O. Hoffm. Habit: Herb. Habitat: Abandoned cultivation, 11002800 m. Voucher: Symes 261 (EA).

Vernonia smithiana Less. Habit: Herb. Habitat: Wooded grassland, 1350-2700 m. Vouchers: Napier 1934 (EA), Symes 27 (EA).

Vernonia syringifolia O. Hoffm. Habit: Shrub. Habitat: Montane rain forest, 20003000 m. Vouchers: SAJIT 006800 \& 006818 (EA, HIB), Webster 8897 (EA).

Vernonia turbinata Oliv. \& Hiern ex Oliv. Habit: Herb. Habitat: Wooded grassland, 1400-2400 m. Voucher: Verdcourt 745 (EA).

Vernonia urticifolia A. Rich. Habit: Shrub. Habitat: Forest margin and bamboo edge, 1400-2800 m. Vouchers: SAJIT Z0084 (EA, HIB), FOKP 11358 (EA, HIB), Mbuni 094 (EA).

Vernonia westermanii Ekman \& Dusén ex Malme Habit: Herb. Habitat: Wooded grassland, 1400-2400 m. Voucher: FOKP 1064 (EA, HIB).

Xanthium pungens Wallr. Habit: Herb. Habitat: River bed, 1600-1800 m, Voucher: FOKP 11302 (EA, HIB).

\section{F127. Caprifoliaceae}

3 Genera, 3 Species

Cephalaria pungens Szabó Habit: Herb. Habitat: Montane and subalpine grassland, 2400-3000 m. Vouchers: FOKP 11316 (EA, HIB), Irwin 136 (EA).

Scabiosa columbaria L. Habit: Herb. Habitat: Heathland and upper land forest, 2200-3550 m. Vouchers: FOKP 11314 (EA, HIB), Webster 8843 (EA).

Valeriana volkensii Engl. Habit: Herb. Habitat: streamside and marshes, 3000$3500 \mathrm{~m}$. Voucher: Mabberley McCall 231 (EA).

\section{F128. Pittosporaceae}

1 Genus, 3 Species

Pittosporum mannii Hook. f. Habit: Shrub. Habitat: Dry evergreen forest, 20002750 m. Vouchers: SAJIT Z0026 (EA, HIB), FOKP 11570 (EA, HIB), Mbuni $307 \& 651$ (EA).

Pittosporum lanatum Hutch. \& E.A. Bruce Habit: Tree. Habitat: Dry evergreen forest, 2100-2850 m. Vouchers: FOKP 11391 (EA, HIB), Dale 672 (EA). 
Pittosporum viridiflorum Sims. Habit: Tree. Habitat: Wooded grassland, 13002500 m. Voucher: SAJIT 007050 (EA, HIB).

\section{F129. Araliaceae}

4 Genera, 8 Species

Cussonia arborea Hochst. ex A. Rich. Habit: Tree. Habitat: Wooded grassland, 1100-2400 m. Voucher: Napier 1980 (EA).

Cussonia spicata Thunb. Habit: Tree. Habitat: Riverine, grassland forest, 14502500 m. Voucher: FOKP 1026 (EA, HIB).

Hydrocotyle mannii Hook. f. Habit: Herb. Habitat: Forest floor, bamboo forest, 600-3370 m. Voucher: Mabberley \& McCall 301 (EA).

Hydrocotyle ranunculoides $\mathbf{L}$. f. Habit: Herb. Habitat: Common in ponds and marshes, 700-2400 m. Voucher: Barnley via Tweedie 2584 (EA).

Polyscias fulva (Hiern) Harms Habit: Tree. Habitat: Wet highland forest, 1400 2310 m. Vouchers: FOKP 1072 (EA, HIB), SAJIT Z0034 (EA, HIB).

Polyscias kikuyuensis Summerh. Habit: Tree. Habitat: Wet upland forest, 1750 2750 m. Voucher: FOKP 1722 (EA, HIB).

Schefflera abyssinica (Hochst. ex A. Rich.) Harms. Habit: Tree. Habitat: Wet upland forest, 1840-2770 m. Vouchers: SAJIT 004790 \& 005092 (EA, HIB).

Schefflera volkensii (Harms) Harms. Habit: Tree. Habitat: Wet or dry upland forest, 1700-2250 m. Vouchers: SAJIT 004785 (EA, HIB), FOKP 1007, 1228, 1892 \& 11388 (EA, HIB), Friis et al. 2530 (EA).

\section{F130. Apiaceae}

14 Genera, 22 Species

Agrocharis melanantha Hochst. Habit: Herb. Habitat: High altitude grassland, 2300-2700 m. Voucher: Thulin \& Tidings 126 (EA).

Agrocharis pedunculata (Baker f.) Heywood \& Jury. Habit: Herb. Habitat: Wooded grassland, 1860-2100 m. Voucher: Napier 1922 (EA).

Alepidea peduncularis Steud. ex A. Rich. Habit: Herb. Habitat: Montane grassland, 1530-3550 m. Vouchers: SAJIT 004810 \& 005105 (EA, HIB), FOKP 11315 (EA, HIB), Mabberley \& McCall 113 (EA), Napper 1503 (EA).

Apium leptophyllum (Pers.) F. Muell. ex Benth. Habit: Herb. Habitat: Disturbed areas, 1550-1660 m. Vouchers: FOKP 1299 \& 11300 (EA, HIB), Mbuni 036 (EA).

Anthriscus sylvestris (L.) Hoffm. Habit: Herb. Habitat: Bamboo zone, 2100-3550 m. Voucher: Mabberley McCall 200 (EA).

Caucalis incognita C. Norman Habit: Herb. Habitat: Grassland, 1530-2550m. Voucher: Symes 514 (EA).

Caucalis pedunculata Baker f. Habit: Herb. Habitat: Disturbed areas, 1550-2160 m. Voucher: Symes 524 (EA). 
Cryptotaenia africana (Hook. f.) Drude Habit: Herb. Habitat: Montane forest, 1600-3000 m. Vouchers: FOKP 1741 (EA, HIB), Irwin 3777 (EA), Mabberley \& McCall 35 (EA), Irving 377 (EA).

Diplolophium africanum Turcz. Habit: Herb. Habitat: Wooded grassland, 13502500 m. Voucher: Kokwaro 2535 (EA).

Haplosciadium abyssinicum Hochst. Habit: Herb. Habitat: Disturbed areas, 21504600 m. Voucher: Thulin \& Tidigs 152 (EA).

Heteromorpha arborescens var. abyssinica (Hochst. ex A. Rich.) H. Wolff Habit: Herb. Habitat: Wooded grassland, 1300-2600 m. Voucher: Thulin \& Tidigs 152 (EA).

Heteromorpha trifoliata (H.L. Wendl.) Eckl. \& Zeyh. Habit: Habitat: Wooded grassland, 1250-2500 m. Vouchers: Mbuni 304 (EA, HIB), FOKP 11567 (EA, HIB).

Oenanthe procumbens $(\mathbf{H}$. Wolff) Norman Habit: Herb. Habitat: Bamboo and highland forest, 1830-3200 m. Vouchers: Mabberley 574 (EA, HIB), Thulin \&Tidigs 234 (EA).

Peucedanum aculeolatum Engl Habit: Herb. Habitat: Montane forest, 19002700 m. Voucher: FOKP 11398 (EA, HIB).

Peucedanum elgonense $\mathbf{H}$. Wolff Habit: Herb. Habitat: Subalpine zone, streamside marshes, 1800-3500 m. Voucher: SAJIT 004797 (EA, HIB).

Peucedanum winkleri H. Wolff Habit: Herb. Habitat: Montane forest, 18003500 m. Voucher: Thulin \&Tidigs 45 (EA).

Peucedanum kerstenii Engl. Habit: Herb. Habitat: Montane forest, 1800-3500 m. Voucher: Bogdan 4995 (EA).

Pimpinella hirtella A. Rich. Habit: Herb. Habitat: Upland grassland, 1800-2850 m. Vouchers: Symes 11345 (EA), Webster 8820 (EA).

Pimpinella lindblomii H. WolffHabit: Herb. Habitat: Upland grassland, 1530-2600 m. Vouchers: FOKP 11695, Mbuni 695 (EA), Lewis 5988 (EA), Symes 755 (EA).

Pimpinella oreophila Hook. f. Habit: Herb. Habitat: Upper montane forest, heath zone, 3000-3550 m. Voucher: SAJIT 005151 (EA, HIB).

Sanicula elata Buch.-Ham. ex D. Don Habit: Herb. Habitat: Bamboo zone, 15003220 m. Vouchers: SAJIT 006902 \& 006906 (EA, HIB), FOKP 1083, 11006 \& 11614 (EA, HIB), Mbuni 614 (EA), Townsend 2384 (EA).

Torilis arvensis subsp. purpurea (Guss.) Thell. Habit: Herb. Habitat: Drier upland forest, 1560-2850 m, Bogdan 5001 (EA).

\section{Acknowledgements}

We are grateful to the National Environment and Management Authority, the Kenya Forest Service and Kenya Wildlife Service for facilitating access permit to undertake botanical research in Cherangani Hills Forest. The authors are indebted to the staff of the Department of Botany, National Museums of Kenya, especially Peris Kamau, Itambo Malombe, Thomas Mwadime Nyange, Mathias Mbale and Kennedy Matheka for their assistance in the course of the research studies. We thank students from the 
University of Chinese Academy of Sciences who tirelessly assisted in data collection. We express our general appreciation to Anne Christine Ochola for reviewing the English version of the text. We are also grateful to the two anonymous reviewers and editors for giving very useful comments on the article. This study was supported by the fund of Sino-Africa Joint Research Center, CAS, China (Y323771W07 and SAJC201322) and the National Natural Science Foundation of China (31800176).

\section{References}

Agnew ADQ (2013) Upland Kenya wild flowers and ferns, third edition. Nature Kenya Publications, Nairobi.

Agnew ADQ, Agnew S (1994) Upland Kenya wild flowers, second edition. East Africa Natural History Society, Nairobi.

APG IV (2016) An update of the Angiosperm Phylogeny Group classification for the orders and families of flowering plants: APG IV. Botanical Journal of the Linnean Society 181(1): 1-20. https://doi.org/10.1111/boj.12385

Beentje HJ, Ndiang'ui N (1988) Ecology of the forests. An ecological and floristic study of the forests of the Taita Hills, Kenya. Utafiti Occasional Papers of the National Museums of Kenya 1: 1-42 Beentje HJ (1994) Kenya trees, shrubs and lianas. National Museums of Kenya, Nairobi.

Beentje HJ (2016) Plant Glossary, an Illustrated Dictionary of Plant Terms ( $\left.2^{\text {nd }} e d n\right)$. Royal Botanic Gardens, Kew.

Blackett H (1994) Forest inventory report number 13 Cherangani hills east, Kipkunurr. KIFCON, Karura forest station, Nairobi.

Blundell M (1987) Collins Guide to the Wild Flowers of East Africa. Collins, London.

Bytebier B, Bussmann RW (2000) The vegetation of mount Nyiru Samburu district, Kenya. A checklist and syntaxonomical survey. Journal of East African Natural History 89(1): 45-71. https://doi.org/10.2982/0012-8317(2000)89[45:TVOMNS]2.0.CO;2

Chong KY, Hugh TW, Corlett RT (2009) A checklist of the total vascular plant flora of Singapore: native, naturalized and cultivated species. Raffles Museum of Biodiversity Research.

Christenhusz MJM, Zhang XC, Schneider H (2011) A linear sequence of extant families and genera of lycophytes and ferns. Phytotaxa 19(1): 7-54. https://doi.org/10.11646/phytotaxa.19.1.2

Filgueiras TS, Brochado AL, Nogueira PE, Gualla GF (1994) Caminhamento-Um método expedito para levantamentos florísticos e qualitativos. Caderno Geociências IBGE 12:39-43.

Fischer E, Rembold K, Althof A, Obholzer J, Malombe I, Mwachala G, Theisen I (2010) Annotated checklist of the vascular plants of Kakamega forest, Western province, Kenya. Journal of East African Natural History 99(2): 129-226. https://doi.org/10.2982/028.099.0205

FAO [Food and Agriculture Organisation] (1997) State of the world's forests. FAO, Rome.

FAO [Food and Agriculture Organisation] (2010) Global forest resources assessment 2010: Main report, Food and Agriculture Organization. FAO Forestry Paper, 163 pp.

Frood D, Calder M (1987) Natural conservation in Victoria study report. Victoria National Parks Association, Melbourne.

Gachathi FN, Johansson SG, Alakoski-Johansson GM (1994) A check-list of indigenous trees and shrubs of Bura, Tana River district Kenya with Malakote, Orma and Somali names. 
Journal of East African Natural History 83(2): 117-141. https://doi.org/10.2982/00128317(1994)83[117:ACOITA]2.0.CO;2

Girma A, Fischer E, Dumbo B (2015) Vascular plant diversity and community structure of Nandi forests, western Kenya. Journal of East African Natural History 103(2): 125-152. https://doi.org/10.2982/028.103.0202

Hall JB, Swaine MD (1981) Distribution and ecology of vascular plants in a tropical rain forest - Forest vegetation in Ghana. Springer Netherlands, Netherlands, 383 pp. https://doi. org/10.1007/978-94-009-8650-3

Heywood VH, Hawksworth DL, Mirk PM, Clarke SD (1997) Information needs in biodiversity assessments, from genes to ecosystems. Biodiversity information needs and options. Centre for Agriculture and Biosciences International, Wallingford, 15-20.

Holmgren P (2006) Global land use area change matrix-input to the fourth Global Environmental Outlook (GEO-4), Forestry department, FAO. Working paper, 134 pp.

IUCN (2013) IUCN Red List of Threatened Species (ver. 2013.2). http://www.iucnredlist.org Luke Q (2005) Annotated checklist of the plants of the Shimba hills, Kwale district, Kenya. Journal of East African Natural History 94(1): 5-120. https://doi.org/10.2982/00128317(2005)94[5:ACOTPO]2.0.CO;2

Lung T, Schaab G (2009) A comparative assessment of land cover dynamics of three protected forest areas in tropical Eastern Africa. Environmental Monitoring and Assessment 161(14): 531-548. https://doi.org/10.1007/s10661-009-0766-3

Lunt ID (1990) Species area curves and growth form spectra for some herb rich woodlands in western Victoria, Australia. Australian Journal of Ecology 15(2): 155-161. https://doi. org/10.1111/j.1442-9993.1990.tb01524.x

MEWNR, RDA (2015) Kenya Biodiversity Atlas, Ministry of Environment, Natural Resources and Regional Development Authorities, Nairobi.

Mitchell N, Schaab G (2008) Developing a disturbance index for five East African forests using GIS to analyse historical forest use as an important driver of current land use/cover. African Journal of Ecology 46(4): 572-584. https://doi.org/10.1111/j.1365-2028.2007.00901.x

Muasya JM, Young TP, Okebiro DM (1994) Plant checklist of Ol ari Nyiro ranch and the Mukutan gorge, Laikipia Kenya. Journal of East African Natural History 83(2): 143-197. https://doi.org/10.2982/0012-8317(1994)83[143:VMAPCO]2.0.CO;2

Nehal AF, Majila BS, Kala CP (2004) Indigenous knowledge systems and sustainable management of a natural resources in a high altitude society in Kumaun Himalaya, India. Journal of Human Ecology (Delhi, India) 16(1): 33-42. https://doi.org/10.1080/09709274.2004.11905713

Ngumbau VM, Nyange M, Dai C, Zhong ZX, Wei N, Malombe I, Hu GW, Wang QF (2017) Adenia angulosa (Passifloraceae), a new species from coastal forests of Kenya and Tanzania. Phytotaxa 313(1): 137-142. https://doi.org/10.11646/phytotaxa.313.1.10

PPG I (2016) A community-derived classification for extant lycophytes and ferns. Journal of Systematics and Evolution 54(6): 563-603. https://doi.org/10.1111/jse.12229

Pratt DJ, Greenway PJ, Gwynne MD (1966) A Classification of East African Rangeland, with an Appendix on Terminology. Journal of Applied Ecology 3(2): 369-382. https://doi. org/10.2307/2401259

Roberts MR (2004) Response of the herbaceous layer to disturbance in North American forests. Canadian Journal of Botany 82(9): 1273-1283. https://doi.org/10.1139/b04-091 
Sayer JA, Zuidema PA, Rijks M (1995) Managing for biodiversity in humid tropical forests.

Commonwealth Forestry Review 74: 282-287.

Stevens PF (2012) Angiosperm phylogeny website. http://www.mobot.org/MOBOT/research/APweb Stewart JJ, Campbell B (2006) Angraecoid Orchids. Species from the African Region. Timber Press, Portland, Oregon.

Synott TJ (1979) A report on the status, importance and protection of montane forests. IPAL technical report number D-2a. UNEPMAB Integrated project in arid lands. UNESCO, Nairobi.

Thijs KW, Roelen I, Musila WM (2013) Field guide to the woody plants of Taita hills, Kenya. Journal of East African Natural History 102(1/2): 1-272.

Tweedie EM (1976) Habitats and check-list of plants on the Kenya side of Mount Elgon. Kew Bulletin 31(2): 227-257. https://doi.org/10.2307/4109170

UNDP (2006) United Nations Development Programme. Community Management of Protected Areas for Conservation (Compact). Community-based initiatives to conserve biodiversity in world heritage landscapes.

UNEP (2009a) Kenya: Atlas of our changing environment, Nairobi. United Nations Environment Programme, Division of Early Warning and Assessment (DEWA).

UNEP, FAO, UNFF (2009b) Vital forest graphics, The United Nations Environment Programme, The Food and Agriculture Organization of the United Nations and The United Nations Forum on Forests secretariat.

Wang X, Feng Z, Ouyang Z (2001) The impact of human disturbance on vegetative carbon storage in forest ecosystems in China. Forest Ecology and Management 148(1): 117-123. https://doi.org/10.1016/S0378-1127(00)00482-5

Wei N, Miyawa DO, David MK, Ngumbau VM, Zhong ZX, Mwachala G, Hu GW, Wang QF (2017) Zehneria longiflora (Cucurbitaceae), a new species from Kenya. Phytotaxa 324(1): 89-94. https://doi.org/10.11646/phytotaxa.324.1.7

Whigham DF (2004) Ecology of woodland herbs in temperate deciduous forests. Annual Review of Ecology and Systematics 35(1): 583-621. https://doi.org/10.1146/annurev.ecolsys.35.021103.105708

Whittaker RH, Niering WA, Crisp MD (1979) Structure, pattern and diversity of a mallee community in south Wales. Vegetatio 39(2): 65-76. https://doi.org/10.1007/BF00052018

Zhou YD (2017) Inventory and diversity of vascular plants in Mount Kenya, East Africa. PhD Thesis, Wuhan Botanical Garden, Wuhan.

Zhou YD, Hu GW, Yan X, Mwachala G, Gituru RW, Wang QF (2016a) Sedum keniense (Crassulaceae), a new species from Mt. Kenya, East Africa. Phytotaxa 261(2): 177-184. https:// doi.org/10.11646/phytotaxa.261.2.7

Zhou YD, Mbuni Y, Hu GW, Yan X, Mwachala G, Wang QF (2016b) Zehneria subcoraicea (Cucurbitaceae), a new species from Kenya. Phytotaxa 277(3): 282-286. https://doi. org/10.11646/phytotaxa.277.3.6

Zhou YD, Liu B, Mbuni Y, Yan X, Mwachala G, Hu G, Wang QF (2017a) Vascular flora of Kenya, based on the flora of tropical East Africa. PhytoKeys 90: 113-126.

Zhou YD, Mbuni Y, Hu GW, Zhong ZX, Yan X, Mwachala G, Wang QF (2017b) Cissampelos keniensis (Menispermaceae), a new species from Mount Kenya, East Africa. Phytotaxa 292(1): 57-64. https://doi.org/10.11646/phytotaxa.292.1.5 